\title{
Taxonomic Revision of Trisetum Section Sibirica (Poaceae: Pooideae: Aveninae)
}

Author(s): Patricia Barberá, Carlos Romero-Zarco, and Carlos Aedo

Source: Systematic Botany, 42(4):1-25.

Published By: The American Society of Plant Taxonomists

URL: http://www.bioone.org/doi/full/10.1600/036364417X696564

BioOne (www.bioone.org) is a nonprofit, online aggregation of core research in the biological, ecological, and environmental sciences. BioOne provides a sustainable online platform for over 170 journals and books published by nonprofit societies, associations, museums, institutions, and presses.

Your use of this PDF, the BioOne Web site, and all posted and associated content indicates your acceptance of BioOne's Terms of Use, available at www.bioone.org/page/terms of use.

Usage of BioOne content is strictly limited to personal, educational, and non-commercial use. Commercial inquiries or rights and permissions requests should be directed to the individual publisher as copyright holder. 
Systematic Botany (2017), 42(4): pp. 1-25

(c) Copyright 2017 by the American Society of Plant Taxonomists

DOI 10.1600/036364417X696564

Date of publication December 18, 2017

\title{
Taxonomic revision of Trisetum section Sibirica (Poaceae: Pooideae: Aveninae)
}

\author{
Patricia Barberá, ${ }^{1,3}$ Carlos Romero-Zarco, ${ }^{2}$ and Carlos Aedo ${ }^{1}$ \\ ${ }^{1}$ Real Jardín Botánico, Consejo Superior de Investigaciones Científicas, Plaza de Murillo, 2, 28014 Madrid, Spain \\ ${ }^{2}$ Departamento de Biología Vegetal y Ecología, Facultad de Biología, Sevilla, Spain \\ ${ }^{3}$ Author for correspondence (pbarbera@rjb.csic.es) \\ Communicating Editor: Ricarda Riina
}

\begin{abstract}
A taxonomic revision of Trisetum sect. Sibirica is presented. We include descriptions and synonyms of each taxon from a study of 450 vouchers from 35 herbaria. Detailed morphometric descriptions, illustrations, distribution maps, identification key, and habitat data are given for each taxon. An identification key for all taxa of Trisetum sect. Sibirica is provided. Morphometric variation of the main characters is shown by box plots. Six names are lectotypified. We recognize six species of Trisetum in the section: T. aeneum, T. bifidum, T. henryi, T. scitulum, T. sibiricum, and T. turcicum. Two infraspecific taxa of T. sibiricum are recognized (T. sibiricum subsp. sibiricum and T. sibiricum subsp. litorale), while T. pauciflorum, T. sikkimense, and T. umbratile are reduced to synonyms of T. sibiricum subsp. sibiricum. Four of the six species of Trisetum sect. Sibirica are endemic to Eastern Asia and New Guinea, while T. turcicum grows in Turkey, the Caucasus, and Northern Iran, and T. sibiricum is widespread from Eastern Europe to Alaska and Canada.
\end{abstract}

Keywords-Asia, Aveninae, grasses, lectotypification, nomenclature, taxonomy.

Trisetum Pers. is a perennial genus of grasses which belongs to the tribe Poeae R. Br. (Tzvelev 1989; GPWG 2001; Soreng et al. 2003, 2007, 2015), and to subtribe Aveninae J. Presl, which comprised 13 genera (Soreng et al. 2015). In recent studies, two new genera have been added to subtribe Aveninae, the genera Trisetopsis Röser \& A. Wölk (Wölk and Röser 2013), and Tzveleviochloa Röser \& A. Wölk (Wölk and Röser 2017). Trisetum, characterized by a bifid, awned lemma and mostly glabrous ovary, is comprised of approximately 70 species that inhabit temperate and cold regions, mainly in the northern hemisphere, but are also found in South America, Australia, and New Zealand. Typically, they live in weedy places, forests, meadows, mountain slopes, and alpine and tundra grasslands (Hultén 1959; Chrtek 1965; Clayton and Renvoize 1986; Randall and Hilu 1986; Watson and Dallwitz 1992; Finot et al. 2004, 2005a). Detailed taxonomical revisions of American taxa, as well as taxa from New Zealand, have been made (Edgar 1998; Finot et al. 2004, 2005a, b); however, only partial revisions exist for Europe and Asia. All the species of $T$. sect. Sibirica are endemic to Asia, two of them reaching to New Guinea and North America.

As in the previous study (Barberá et al. 2017), we continue the traditional separation of the two genera based on their perennial (Trisetum) versus annual (Trisetaria) life cycles (e.g. Chrtek 1965; Rechinger 1970; Tzvelev 1976; Pignatti 1982; Finot et al. 2004, 2005a, b); therefore, the proposal of Quintanar and Castroviejo (2010) to conserve Trisetum against Trisetaria is followed.

Trisetum has been traditionally divided into two sections: $T$. sect. Trisetum, with lax, open panicles and culms glabrous below the inflorescences, and T. sect. Trisetaera Asch. \& Graebn., with dense, spiciform panicles, and culms pilose below the inflorescences. A review of the history of the genus in America and Europe can be found in Finot et al. (2005b) and Barberá et al. (2017).

Chrtek (1965) deeply studied the European species of Trisetum, dividing them into four subgenera (T. subg. Trisetum, $T$. subg. Distichotrisetum, T. subg. Glaciotrisetum, and T. subg. Graciliotrisetum), mainly based on the anatomy of the leaves and roots. At the same time, Chrtek divided the subgenus Trisetum in five sections (T. sect. Trisetum, T. sect. Trisetaera, $T$. sect. Rigida, T. sect. Hispanica, and T. sect. Carpatica). Trisetum sect. Trisetum is characterized by its lax panicles and leaves with the sclerenchyma clearly developed in young leaves. This section, defined by Chrtek (1965), includes the species of $T$. sibiricum group.

Chrtek (1968) described one new species from Nepal, Sikkim, southeastern Tibet and China, Trisetum scitulum, and divided sect. Trisetum into two new series: T. ser. Trisetum, and T. ser. Sibirica. Trisetum ser. Trisetum (including T. flavescens, T. turcicum from Turkey and Caucasus, T. thospiticum from Van Lake in Turkey, the Chinese species T. henryi, and T. scitulum) was characterized by its geniculate awns, sometimes twisted at the lower part. On the other hand, Trisetum ser. Sibirica (including T. sibiricum, the Central Asian T. altaicum, and two Himalayan species T. aeneum, and also T. micans with some doubts) was diagnosed by its recurved and not geniculate awns, not clearly twisted at the lower part. About the Eastern Asiatic species T. bifidum, Chrtek (1968) indicated that it is a species close to sect. Trisetum, but it is not included in either series.

Tzvelev (1976) in his treatment of Trisetum for the Soviet Union recognized three sections (T. sect. Rigida, T. sect. Trisetum, and T. sect. Trisetaera). He simplified Chrtek's classification, not accounting for the anatomical characters used by Chrtek (1965). Tzvelev characterized the sections by the type of growth of underground and vegetative shoots, the leaf disposition, the panicle density, and the rachilla and callus hair length. He included seven species and eight subspecies within T. sect. Trisetum (T. ciliare, T. turcicum, T. sibiricum subsp. sibiricum, T. sibiricum subsp. litorale, T. sibiricum subsp. umbratile, T. flavescens subsp. flavescens, T. flavescens subsp. parvispiculatum, T. flavescens subsp. tatricum, T. alpestre subsp. alpestre, T. alpestre subsp. glabrescens, T. altaicum, and T. agrostideum), and did not discuss the series.

Later, Probatova (1978) in her study of the genus from Caucasus arranged Trisetum sect. Trisetum in four subsections: the monotypic T. subsect. Carpatica (Chrtek) Probat. (including T. ciliare), subsect. Trisetum (including T. flavescens s. l., T. parvispiculatum and T. alpestre), subsect. Sibirica (Chrtek) Probat. (including T. sibiricum and T. turcicum), and subsect. Agrostidea Probat. (including T. altaicum and T. agrostideum). The different subsections are characterized by the type of growth of underground and vegetative shoots, lemma apex, callus, awn and palea keel hair length and anther length.

Two other relevant recent floristic accounts cover species of Trisetum subsect. Sibirica. Wu and Phillips (2006), in their 
treatment of Flora of China, did not recognize any sections, and considered the subspecies of $T$. sibiricum as species. Enushchenko (2011), in his revision of Trisetum in the Northern Asia, also considers the subspecies of T. sibiricum as species, but recognized the subsection Sibirica of Probatova (1978).

Therefore, the main points of controversy of this group of Trisetum concern the divisions of $T$. sibiricum, sometimes divided into many taxa, and the circumscription and rank of the series or subsection Sibirica. In this work, Probatova criteria (1978) of classification and our unpublished molecular data are followed to characterize Trisetum sect. Sibirica.

We present a taxonomic revision of Trisetum sect. Sibirica based on review of herbarium material, as part of a monograph of Trisetum in Eurasia. The synopses mentioned above were all partial works, not providing a detailed view of the entire section. The present work provides a comprehensive study of this section.

\section{Materials ANd Methods}

This revision is based on the study of 450 herbarium specimens from the following herbaria: AAH, B, BEOU, BM, C, E, F, FI, G, GB, GH, GOET, H, IFP, JE, K, KYO, KUN, L, LE, LIV, M, MO, MPU, MW, NY, P, PE, PH, PR, PRC, RO, S, TI, UPS, US, W, and WU (acronyms according to Thiers 2017). Photos of specimens from CDBI, HHBG, HNWP, PE, and QTPM were also examined on the website of the Chinese Virtual Herbarium $(\mathrm{CVH}$; www. cvh.org.cn). Specimens cited in the text are listed in Appendix 1.

Ninety specimens were used for the morphometric analyses, as operational taxonomic units (OTUs), selected to represent as far as possible the geographic range and morphological variability of the taxa. Fifty-nine quantitative characters were recorded using a Mitutoyo CD-15DCX digital vernier caliper on 20 specimens of each species when available. Commonly used characters in Trisetum taxonomy were selected, as well as those observed to be variable and diagnostic in herbarium specimens. Spikelet measurements were taken on the distal spikelet from the longest branch of the second node of the panicle; floret measurements were taken from the proximal branches. Leaf-blade width was measured one $\mathrm{cm}$ above the ligule insertion. The rachilla segment between first and second flower is also referred to as "rachilla." Each character was analyzed for its minimum, maximum, and lower and upper quartiles, using STATISTICA package (www.statsoft.com). Quantitative and qualitative characters are also used in the identification key and descriptions. Minimum and maximum values are noted in brackets and the lower and upper quartile values (between the 25 th and 75th percentiles) are noted outside. A combination of morphological characters was employed to distinguish species. Transverse sections of top leaf-blades were prepared by hand, stained with Fasga (Tolivia and Tolivia 1987), and photographed with a Nikon SMZ1000 optical microscope. For leaf anatomy, the terminology defined by Ellis $(1976,1979)$ was used.

Additional data on the habitat, distribution, and chromosome numbers were checked from literature and collection labels. Chromosome numbers were summarized from the literature, but the extant vouchers, if any, were not revised. The list of numbered collections, examined specimens, and coordinates used to produce distribution maps were generated by a Microsoft ACCESS database (Microsoft, Seattle, Washington). Species distribution maps were made using ArcGis v. 9.3 (ESRI 2008). The material studied is listed under each species in the taxonomic treatment below. The data for establishing coordinates was completed in almost all cases, using www.geonames.org database.

\section{RESUlts}

Morphology-HABIT AND STEM-Trisetum sect. Sibirica are perennial grasses, short rhizomatous, sometimes with stoloniferous rhizomes (in T. sibiricum subsp. sibiricum), with well-isolated shoots or loosely tufted habit, normally densely tufted in T. bifidum.

The stems are straight, and usually shorter than $90 \mathrm{~cm}$, except T. henryi, which can reach more than $150 \mathrm{~cm}$, followed by T. sibiricum subsp. sibiricum, which can rarely also reach these sizes. Trisetum sibiricum subsp. sibiricum is the most widespread species of the section, and the taxon with the greatest variation in culm length, $(12.7-) 57 \mathrm{~cm}$ to $100(-150) \mathrm{cm}$ long.

The species of this section present extravaginal growth in the lower and upper nodes, except $T$. henryi, whose upper ones are enclosed by the sheaths. The culms are glabrous in all the species (rarely pubescent on the upper part in T. turcicum), with (2-)3-6(-7) nodes. The culm internodes are elongated and separated along the culms in all the species, although sometimes T. sibiricum subsp. litorale has shorter internodes concentrated in the lower part.

LeAves - As in the rest of the genus Trisetum, the indumentum, shape, and size of ligules, leaf-sheaths, and leafblades are variable characters in the same plant, depending on whether they are the basal or the top culm-leaf. Notable heterophylly occurs between young and mature leaf-blades in genus Trisetum, as well as in the closely related genus Koeleria (Quintanar and Castroviejo 2013; Barberá et al. 2017).

LEAF-SHEATHS - Basal leaf-sheaths are glabrous to slightly, rarely densely, pubescent. Top culm leaf-sheaths are shorter than their respective internodes, rarely longer in T. bifidum, $T$. henryi, and T. scitulum; they are always glabrous in T. aeneum, T. bifidum, and T. scitulum, sometimes pubescent in T. henryi, T. sibiricum, and T. turcicum.

Sheath margins are glabrous, except for those of T. bifidum, T. henryi, and, sometimes, T. sibiricum subsp. sibiricum and $T$. turcicum, which are ciliate.

LEAF-BLADES - The species of Trisetum sect. Sibirica have flat and non-rigid leaf-blades. The indumentum of leaf-blades has traditionally been used as a distinctive character for taxa delimitation (Tzvelev 1976; Jonsell 1980). However, leaf-blade features (mainly indumentum and width) have been considered less important than those linked to spikelet morphology for the taxonomy of the widespread species, due to the high variability of leaf-blade traits. Most of the species of this section have leaves varying from glabrous to pubescent adaxially and abaxially, usually with short hairs on the margins.

The species of Trisetum, including sect. Sibirica, are mesophytic or xerophytic grasses. While the abaxial surface of the leaf-blades often has a regular surface, the adaxial one has ribs of varying depth (Metcalfe 1960; Watson and Dallwitz 1992). In xerophytic species of Trisetum sect. Sibirica, the depth of the intercostal zones and associated ribs is more pronounced, and the sclerenchyma girders and strands are well-developed. The midrib is not readily distinguishable in all of the species of the section, being conspicuously marked in T. henryi, and sometimes in T. sibiricum and T. turcicum. Among the species of this section, all the vascular bundles are accompanied by more or less developed sclerenchyma girders and strands that attach to both the abaxial and adaxial sides of the leaf-blade. Trisetum sibiricum subsp. litorale, and sometimes T. sibiricum subsp. sibiricum, have vascular bundles with well-developed sclerenchyma strands abaxially and small strands adaxially, and nerves with no sclerenchyma present or with small girders in both sides. Trisetum sibiricum subsp. litorale, and sometimes $T$. sibiricum subsp. sibiricum, are mesophytic grasses, which explains their less developed sclerenchyma girders and strands. Trisetum aeneum, T. bifidum, T. henryi, T. scitulum, T. turcicum, and usually T. sibiricum subsp. sibiricum have well-developed sclerenchyma girders, anchor-shaped or relatively wide and deep bands, and leaf margins with cap of sclerenchyma. 
LigulEs - The ligules are membranous, with a truncate, denticulate to laciniate apex, glabrous or pubescent abaxially in T. bifidum, T. henryi, and T. sibiricum subsp. sibiricum, usually with some hairs on the upper part. The shape and size of ligules are variable, with those of basal leaves being shorter than those of top culm leaves. The ligule measurements used in this study were taken from the top culm leaf-blades. Trisetum scitulum has the longest ligules $(2.2-3.5 \mathrm{~mm})$, while T. bifidum has the shortest [0.4-1.1(-1.6) $\mathrm{mm}]$.

INFLORESCENCES-Inflorescences are usually lax panicles, but are sometimes dense in T. aeneum, T. sibiricum subsp. litorale, and T. turcicum, oblong to elliptic or lanceolate in outline, and sometimes ovate in T. sibiricum subsp. litorale. Trisetum henryi has the longest panicles [(16-)19-23(-28) cm long], followed by T. bifidum and T. sibiricum subsp. sibiricum $[(8.5-) 11-19(-27) \mathrm{cm}$ and (5-)11.7-16(-23) cm long, respectively], whereas T. sibiricum subsp. litorale has the smallest ones [(3.3-)5-7.4(-9) $\mathrm{cm}$ long]. Trisetum henryi and T. scitulum are characterized by having the longest basal branches, while T. aeneum, T. sibiricum subsp. litorale, and T. turcicum have the shorter ones. Figure 1A-B.

Trisetum sect. Sibirica usually has a glabrous rachis, but is sometimes slightly pubescent on the upper part in T. bifidum, T. sibiricum, and T. turcicum, with hairs up to $0.1 \mathrm{~mm}$ long.

SPIKELETS-Spikelet length in Trisetum sect. Sibirica varies according to the lemma length, spikelets being longest in T. scitulum, T. turcicum, and T. bifidum; having a broad range of length in T. sibiricum subsp. sibiricum; and being slightly shorter than average in T. sibiricum subsp. litorale, T. aeneum, and T. henryi (Fig. 1C). The awn is not included in the length of the spikelet.

Rachilla segments are always pubescent, with fairly constant hair length at the species level, although it is not useful as a diagnostic character in this section. The number of florets is usually $2-3$ per spikelet, sometimes 1 or 4 in T. sibiricum subsp. sibiricum, and also 4 in T. bifidum.

GLumes - In the section Sibirica, Trisetum bifidum glumes are always markedly unequal [ratio of lower glume length/upper glume length $=(0.11-) 0.48-0.59(-0.67)]$, also pronounced in $T$. henryi, and sometimes in $T$. sibiricum. The glumes are less markedly unequal in the rest of species, being subequal in T. aeneum, and rarely in T. sibiricum (Fig. 1D). Lower and upper glumes are acuminate, sometimes acute or long acuminate, with some short hairs from the middle part to the top of the main nerve and along the margins. The lower glume is almost always shorter than the upper one, as indicated before, with a single nerve extending to the tip. The upper glume always has three nerves, the central one reaching the tip, the lateral ones extending to the middle or the upper half.

LEMMA - The lemma of the species of Trisetum sect. Sibirica, as well as that of the rest of the genus, is characterized by having a dorsal awn and a bifid apex with two apical teeth normally ending in two aristules separated by a more or less deep sinus. Aristules are the intermediate nerves protruding beyond each tooth apex (Nicora 1978; Koch 1979; Finot et al. 2006). In this section, the aristules of T. scitulum are longer than the rest of species, the ones of T. bifidum having a broader range of their length [0.5-1.3 mm, and (0.1-)0.6-1(-1.3) mm long, respectively] (Fig. 1G). The length of the lemma, without taking the awn into account, is longer in T. turcicum, T. scitulum, and T. bifidum [6.5-8.7 $\mathrm{mm},(6.5-) 7.4-7.8(-8.4) \mathrm{mm}$, and (6-)6.5-7.5(-8.2) $\mathrm{mm}$ long, respectively], having a broader range of its length in T. sibiricum subsp. sibiricum [(4-)5.4-6.8 (-7.8) $\mathrm{mm}$ long], and T. sibiricum subsp. litorale and T. henryi the shorter ones [(3.8-)4.7-5.7(-6.2) $\mathrm{mm}$ and (4.2-)4.8-6.1 $\mathrm{mm}$ long, respectively]. The lemma is laterally compressed, elliptic to oblong or lanceolate, golden brown or brownish to yellowish at maturity, punctate to scabridulous, with short hairs from the central to the upper part of the midrib (the hairs being slightly longer in T. bifidum).

Callus - The callus is elliptic to rounded, or sometimes oblong. The callus is always glabrous in Trisetum scitulum and T. henryi, and sometimes in T. sibiricum and T. turcicum, while the rest of species of the section have short and scattered or sparse indumentum. Trisetum bifidum is the species with the longest callus hairs (0.3-0.7 mm long).

PALEA - The palea disposition and its shape are the same as in the rest of the species of the genus (Barberá et al. 2017). In this section, the palea of T. turcicum is longer than the rest of species [(5-)5.7-7(-7.2) mm long]. The length of both the palea and the lemma is a good taxonomic character for some species, being always markedly unequal in T. bifidum, and T. scitulum, with the palea reaching to about half the length of the lemma [ratio of palea length/lemma length $=(0.49-) 0.55-0.64(-0.73)$, and 0.6-0.68, respectively]. Trisetum aeneum, T. henryi, and T. turcicum have a slightly less marked unequal difference between these structures [ratio of palea length/lemma length $=(0.61-) 0.7-0.82$, $0.67-0.82(-0.86)$, and $0.74-0.84(-0.93)$, respectively]; they are more variable in T. sibiricum [(0.63-)0.77-0.85(-1)] (Fig. 1E).

LODICUlEs-As in the rest of the genus Trisetum, the two lodicules flank the dorsal sides of the ovary or caryopsis, having a more or less oblanceolate shape. The apex is irregular to regularly lobulated (2-3-lobate), rarely laciniate or denticulate, and always glabrous.

Awn-The length and shape of the awn are important taxonomic characters for the classification of this section. The awn shape is basally recurved and usually slightly twisted near the base, although T. turcicum and T. scitulum has geniculate awns below the middle, and a twisted column up to the geniculate part. Trisetum scitulum has significantly longer awns [(10.5-)13-15 mm long], but the length does not differ greatly in the rest of the species of the section (Fig. 1F). The awn is dorsal, inserted near the middle of the lemma, except T. bifidum, a species with the awn always inserted closer to the apex.

STAMENS-Anther length is a good diagnostic character, being fairly constant within the species level. Trisetum turcicum is the species with the longest anthers [(3-)3.3-4 $\mathrm{mm}$ long], followed by $T$. sibiricum and T. henryi $[(1.4-) 2-2.5(-3) \mathrm{mm}$ long]; the rest of the species of the section have relatively short ones [(0.6-)1-1.3(-1.6) mm long] (Fig. 1H).

OVARY AND CARYOPSIS-The ovary and caryopsis are very similar in all species, being always glabrous. The mature caryopsis is narrowly elliptic to oblong in shape, narrowly elliptic to elliptic in transverse section, not sulcate, with puctiform hilum. The endosperm is soft-liquid.

\section{TAXONOMIC TREATMENT}

TRISETUM sect. SIBIRICA (Chrtek) Barberá, stat. nov. T. ser. sibirica Chrtek, Acta Univ. Carol., Biol. 1967: 107. 1968. T. subsect. Sibirica (Chrtek) Prob., Novosti Sist. Vyssh. Rast.15: 20. 1979.-TYPE: T. sibiricum Rupr.

Herbs shortly rhizomatous, rarely with stoloniferous rhizomes; panicles from lax to dense; spikelets goldish-brown, rarely pale yellowish; callus glabrous or with short hairs up to $0.7 \mathrm{~mm}$ long; awns recurved or basally slightly twisted, not or rarely geniculate. 
A

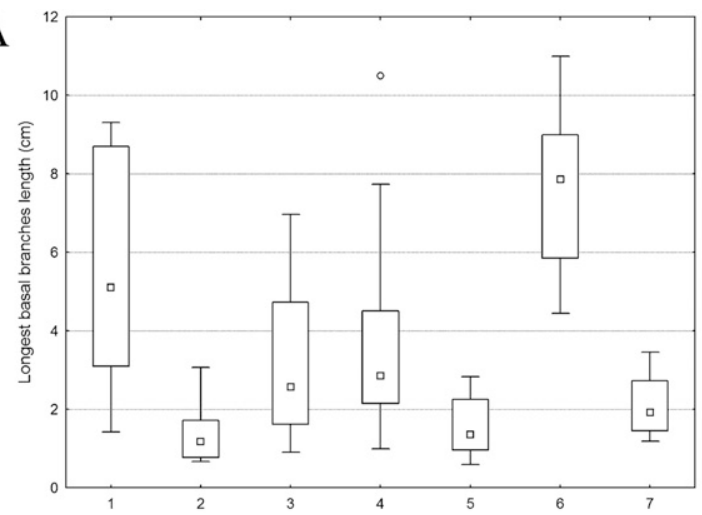

C

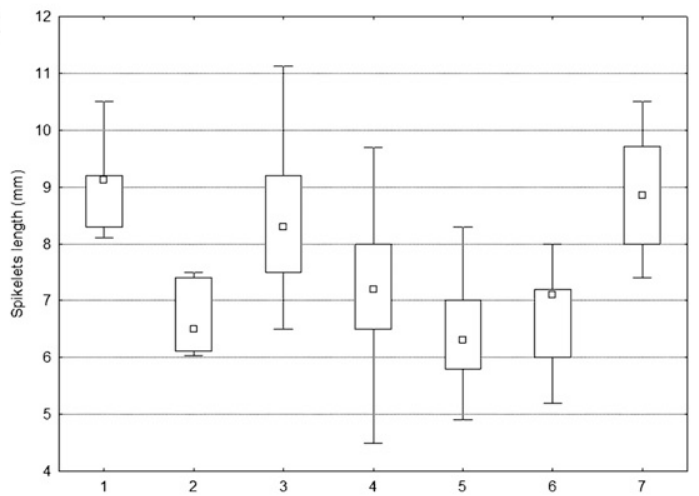

E

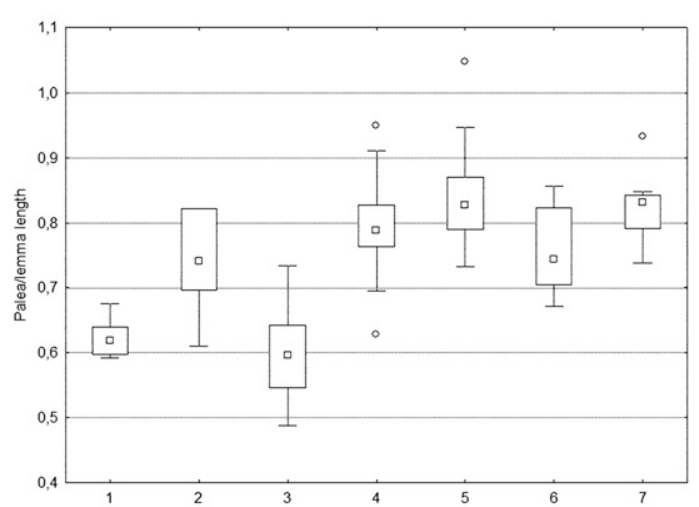

G

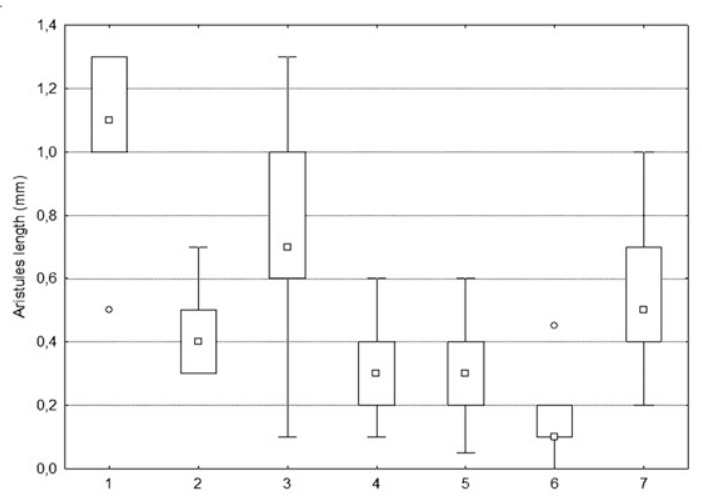

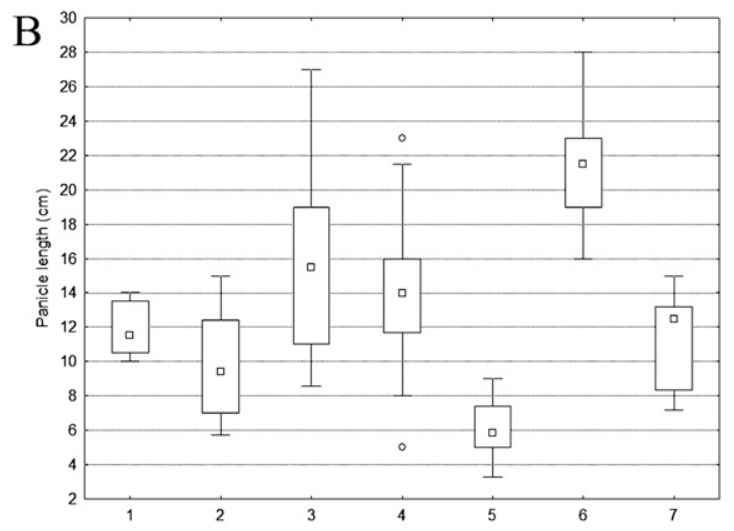

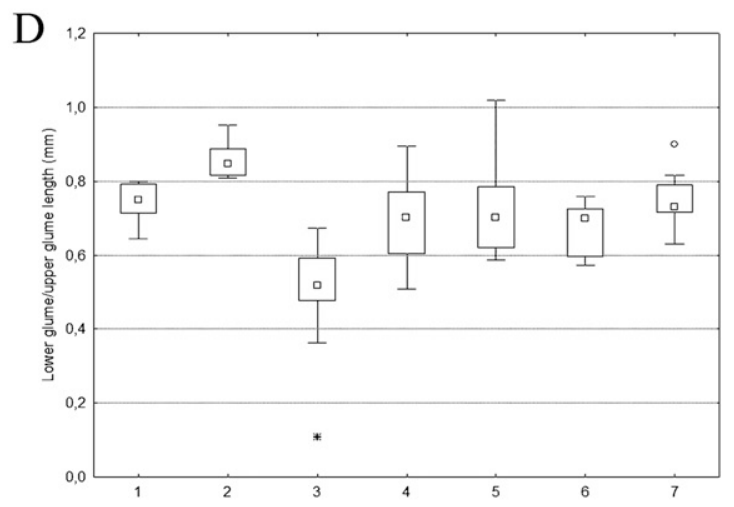

F

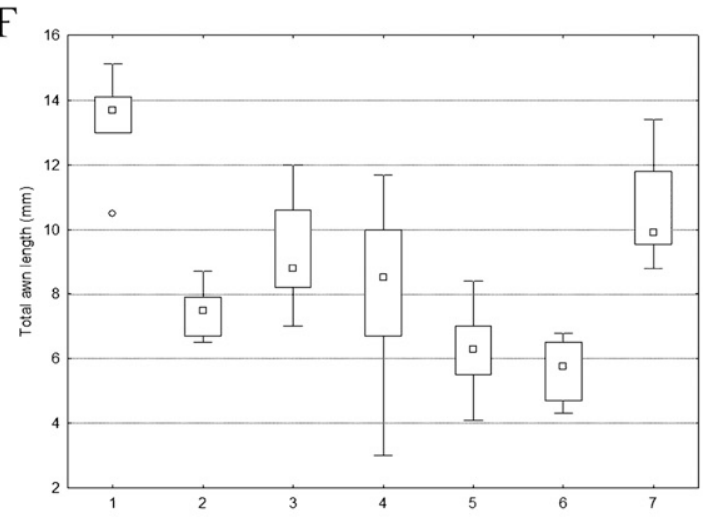

$\mathrm{H}$

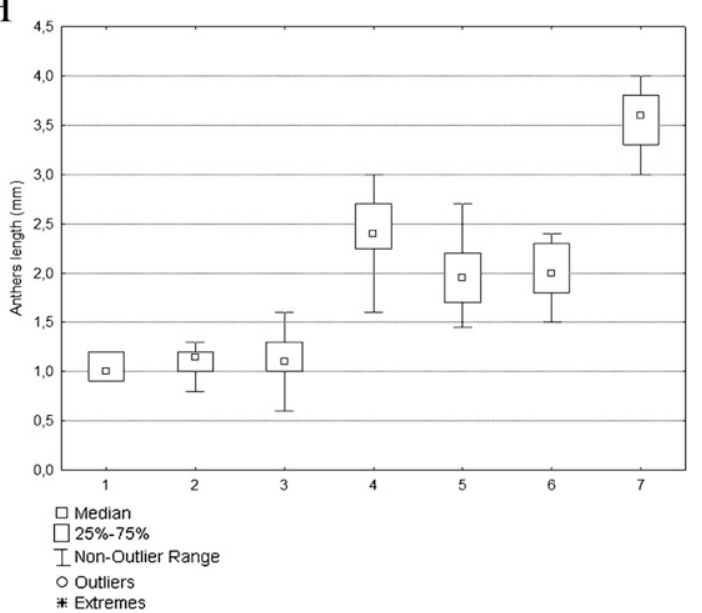

FIG. 1. Box plots of a selection of studied variables. A. Longest basal branches length. B. Panicle length. C. Spikelets length. D. Lower glume/upper glume length. E. Palea/lemma length. F. Total awn length. G. Aristules length. H. Anthers length. Numbers along the x-axis correspond to the studied taxa: 1. T. scitulum. 2. T. aeneum. 3. T. bifidum. 4. T. sibiricum subsp. sibiricum. 5. T. sibiricum subsp. litorale. 6. T. henryi. 7. T. turcicum. 


\section{Key to the Taxa of Trisetum Section Sibirica}

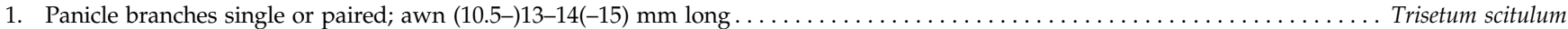

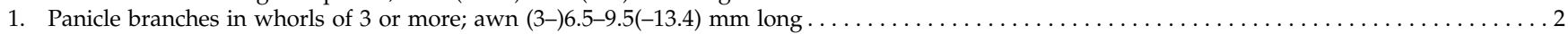

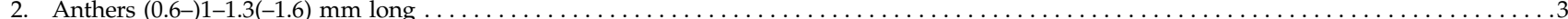

3. Panicle dense; glumes subequal [ratio of lower glume length/upper glume length $=(0.8-) 0.82-0.89(-0.95)]$; lemma $5.6-6(-6.6) \mathrm{mm}$ long; ratio of palea length/lemma length $=(0.61-) 0.7-0.82 ;$ callus hairs $0.1-0.3 \mathrm{~mm}$ long $\ldots \ldots \ldots \ldots \ldots \ldots \ldots \ldots \ldots$ Trisetum aeneum

3. Panicle lax; glumes unequal [ratio of lower glume length/upper glume length $=(0.11-) 0.48-0.59(-0.67)]$; lemma $(6.1-) 6.6-7.6(-8.2) \mathrm{mm}$ long; ratio of palea length/lemma length $=(0.49-) 0.55-0.64(-0.73)$; callus hairs $0.3-0.7 \mathrm{~mm}$ long $\ldots \ldots \ldots \ldots \ldots$ Trisetum bifidum

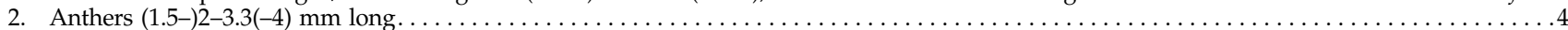

4. Top culm leaf-blades $17.5-29.5 \mathrm{~cm}$ long, reaching to the panicle, with the central nerve conspicuously marked; lemmas pale yellowish or

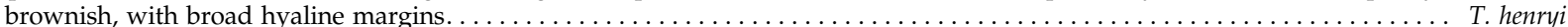

4. Top culm leaf-blades (1.5-)5.6-13(-34.3) cm long, rarely reaching to the panicle, with the central nerve similar to the lateral ones; lemmas golden

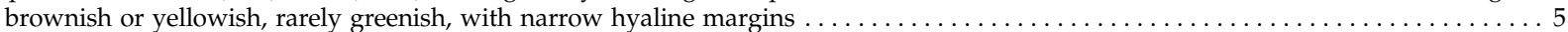

5. Awn geniculate, slightly twisted to twisted near the base; anthers $(3-) 3.3-4 \mathrm{~mm}$ long.................... Turcicum

5. Awn slightly recurved, sometimes slightly twisted at the base; anthers $(1.5-) 2-2.5(-3) \mathrm{mm}$ long $\ldots \ldots \ldots \ldots \ldots \ldots \ldots \ldots \ldots \ldots \ldots \ldots \ldots$ 6. Herb (12.7-)57-100(-146) cm high; panicles (5-)11.7-16(-23) $\mathrm{cm}$ long ....................... sibiricum subsp. sibiricum 6. Herb (12.8-)23.8-37.7(-58.4) cm high; panicles (3.3-)5-7.4(-9) cm long........................ T. sibiricum subsp. litorale

1. Trisetum SIBIRICUm Rupr., Beitr. Pflanzenk. Russ. Reiches 2: 65. 1845. Avena ruprechtii Griseb. in Ledeb., Fl. Ross. 4(13): 418. 1852. Trisetum ruprechtii (Griseb.) Steud., Syn. Pl. Glumac. 1: 226. 1854. Trisetum flavescens var. sibiricum (Rupr.) Ohwi, Bot. Mag. (Tokyo) 45: 192. 1931. Trisetum bifidum subsp. sibiricum (Rupr.) T. Koyama, Grass. Jap. Neighb. Reg.: 533. 1987. Trisetaria sibirica (Rupr.) Banfi \& Soldano, Atti Soc. Ital. Sci. Nat. Mus. Civico Storia Nat. Milano 135: 385. 1996.-TYPE: RUSSIA. Bashkortostan Republic, Belaja river, $55^{\circ} 53^{\prime} \mathrm{N} 53^{\circ} 36^{\prime} \mathrm{E}$, F. J. I. Ruprecht s. $n$. (lectotype, designated by Tzvelev (1976: 262): LE!).

Herb (12.7-)28.8-79(-146) cm high, not or slightly tufted, shortly rhizomatose, sometimes with stoloniferous rhizomes, culm (0.45-)0.9-1.3(-2) mm diam, glabrous; nodes (2-)3-5(-7), separated along the culm, sometimes concentrated in lower part of the culm, exposed or enclosed by the sheaths, glabrous. Basal leaf-sheaths glabrous to pubescent, with hairs $0.03-$ $0.2(-1.8) \mathrm{mm}$ long, frayed into fibers, yellowish to brownish; basal leaf-blades (1.2-)2.7-7(-17.5) cm long $\times(2-) 2.5-$ $3.6(-7.7) \mathrm{mm}$ wide, flat, rolled when dried, glabrous to pubescent, mostly adaxially, with hairs $0.05-0.8(-1.2) \mathrm{mm}$ long, greenish to greyish, sometimes brownish; top culm leaf-sheaths (4.2-)7.8-17(-25) cm long, shorter than the internodes, glabrous to pubescent on the upper part, without cilia on the margins, rarely with; top culm leaf-blades (1.5-)5.2-13(-34.3) $\mathrm{cm}$ long $\times$ (2.5-)3.8-6(-11) mm wide, flat, usually parallel to the culm, rarely extending to the panicle, with the central nerve similar to the lateral ones, from glabrous to pubescent abaxially and adaxially, with hairs up to $1.7 \mathrm{~mm}$ long along the nerves and margins, greenish to greyish, rarely brownish; inner collar region glabrous, usually with hairs (0.05-)0.2-1(-2.2) mm long externally and on the margins; ligules (0.5-)1.3-2(-2.7) mm long, denticulate to laciniate, glabrous to slightly pubescent abaxially and on the apex, with hairs $0.1-0.3(-1) \mathrm{mm}$ long. Basal node of the panicle glabrous. Panicles (3.3-)6-15(-23) cm long $\times$ $(1.2-) 2-4(-10) \mathrm{cm}$ wide, elliptic to lanceolate in outline, sometimes ovate or oblanceolate; rachis glabrous, sometimes slightly pubescent, with hairs up to $0.1 \mathrm{~mm}$ long; branches in whorls of 3 or 6 , longest basal branches $(0.6-) 1.3-3.5(-10.5) \mathrm{cm}$ long. Spikelets (4.5-)6-7.6(-9.7) mm long $\times(1.3-) 2.2-3.4(-5.5) \mathrm{mm}$ wide, (1)2-3(4)-flowered; pedicels (0.5-)2-5.2(-10.5) $\mathrm{mm}$ long, glabrous to slightly pubescent, with hairs up to $0.2 \mathrm{~mm}$ long. Glumes unequal, rarely subequal [ratio of lower glume length/upper glume length $=(0.5-) 0.6-0.77(-1)]$; lower glume (2.5-)3.3-4.4(-5.6) mm long $\times(0.4-) 0.8-1(-1.4) \mathrm{mm}$ wide, narrowly to broadly lanceolate [ratio of lower glume width/lower glume length $=(0.15-) 0.2-0.28(-0.35)]$, acuminate, sometimes acute or long acuminate, 1-nerved, glabrous, with hairs up to $0.15 \mathrm{~mm}$ long on the midrib and margins, greenish on the central part, laterals yellowish or sometimes purplish; upper glume (3.7-)5-6(-7.8) mm long $\times(1-) 1.7-2$ $(-2.5) \mathrm{mm}$ wide, elliptic to oblanceolate [ratio of upper glume width/upper glume length $=(0.25-) 0.3-0.37(-0.5)]$, acuminate to long acuminate, sometimes acute, 3-nerved, glabrous, with hairs up to $0.2 \mathrm{~mm}$ long from the middle to the upper part of the midrib, greenish on the central part, surrounded by yellowish, sometimes purplish; rachilla segments between first and second floret (0.6-)1-1.5(-2) mm long, with hairs (0.2-) 1-1.4(-2.2) mm long; rachilla segments to sterile floret (0.5-) 1-1.5(-2.2) mm long, with hairs (0.2-)0.5-0.7(-1.3) $\mathrm{mm}$ long. Lemmas (3.8-)5.2-6.2(-7.8) $\mathrm{mm}$ long $\times(1-) 1.5-1.8(-2) \mathrm{mm}$ wide, elliptic to oblong, sometimes lanceolate [ratio of lemma width/lemma length $=(0.2-) 0.25-0.33(-0.4)]$, scabridulous, with scattered hairs up to $0.1 \mathrm{~mm}$ long on the upper part of the midrib, golden brownish or yellowish, rarely greenish, with narrow hyaline margins; apical teeth $(0.07-) 0.2-0.5(-1) \mathrm{mm}$ long, with aristules (0.05-)0.2-0.4(-0.6) mm long; awn (3-)6.2-9(-11.7) $\mathrm{mm}$ long, inserted (2.2-)2.8-3.7(-4.4) $\mathrm{mm}$ from the base [ratio of awn insertion from the base length/ lemma length $=0.5-0.6(-0.78)]$, slightly recurved and sometimes slightly twisted at the base, with adpressed hairs up to $0.05 \mathrm{~mm}$ long; callus $0.2-0.3 \mathrm{~mm}$ long, elliptic to orbicular, glabrous or with hairs $0.1-0.2(-0.3) \mathrm{mm}$ long. Paleas (3.3-)4-5(-6.7) $\mathrm{mm}$ long $\times(1-) 1.3-2 \mathrm{~mm}$ wide [ratio of palea length/lemma length $=(0.63-) 0.77-0.85(-1)]$, elliptic, rarely oblanceolate, margins with scattered hairs on the upper part, rarely glabrous; teeth $0.1-0.3(-0.4) \mathrm{mm}$ long, with short hairs. Lodicules (0.4-)0.6-0.8(-1.1) $\mathrm{mm}$ long. Anthers (1.5-)2-2.5(-3) mm long. Ovary (0.3-)0.5$0.8(-1.3) \mathrm{mm}$ long. Caryopsis $2.7-3.7(-4) \mathrm{mm}$ long $\times 0.5-$ 1(-1.2) mm wide.

a. subsp. SIBIRICUM

Avena rufescens Pančić, Fl. Serbiae, Add.: 238. 1884. Trisetum rufescens (Pančić) Adamovic, Index Sem. Hort. Bot. Belgradensi 1903, n. v.-TYPE: SERBIA. Vlasina lake, $42^{\circ} 42^{\prime} \mathrm{N}$ 202'ㄹ, Aug 1880, J. Pančić 1150 (lectotype, here designated: BEOU-13316 (image!)).

Avena sikkimensis Hook. f., Fl. Brit. India 7: 280. 1897. Trisetum sikkimense (Hook. f.) Chrtek, Acta Univ. Carol., Biol. 1967: 104. 1968.-TYPE: INDIA. Sikkim, Lachoong Valley, $27^{\circ} 45^{\prime} \mathrm{N} 88^{\circ} 30^{\prime} \mathrm{E}, 9$ Jun 1849, J. D. Hooker s. n. (lectotype, here designated: K-808691!; possible isolectotypes: E-393839 
(image!), GOET!, K-32272!, K-32273!, M-210837!, P-2255848!, P-3351084!, P-3351085!, S!).

Trisetum homochlamys Honda, Bot. Mag. (Tokyo) 43: 293. 1929.-TYPE: JAPAN. Hokkaido Island, Hidaka Province, Samani distr., foot of Mt. Apoi, $42^{\circ} 6^{\prime} \mathrm{N} 143^{\circ} 1^{\prime} \mathrm{E}, 1928$, T. Nakai s. n. (holotype: TI-21896 (image!)).

Trisetum flavescens f. senanense Ohwi, Bot. Mag. (Tokyo) 45: 192. 1931.-TYPE: JAPAN. Honshu Island, Nagano, Senjo Mountain, $35^{\circ} 43^{\prime} \mathrm{N} 138^{\circ} 11^{\prime} \mathrm{E}$, 28 Aug 1925, J. Ohwi s. n. (holotype: KYO-78671 (image!)).

Trisetum sibiricum var. umbratile Kitagawa, Rep. Inst. Sci. Res. Manchoukuo 4: 77. 1940. T. umbratile (Kitagawa) Kitagawa, J. Jap. Bot. 31: 302. 1956. Trisetum sibiricum subsp. umbratile (Kitagawa) Tzvelev, Spisok Rast. Gerb. Fl. S.S.S.R. Bot. Inst. Vsesojuzn. Akad. Nauk 18: 6. 1970.-TYPE: CHINA. Manchuria, Jilin Province, Jingyue Lake, $43^{\circ} 42^{\prime} \mathrm{N} 126^{\circ} 12^{\prime} \mathrm{E}, 6 \mathrm{Jul}$ 1939, M. Kitagawa s. n. (holotype: TI-21898 (image!)).

Trisetum pauciflorum Keng, Fl. Tsinling. 1(1): 441. 1976.-TYPE: CHINA. Shaanxi, Xianyang, 34²0'N 108 42’E, 29 Jul 1952, K. T. Fu 5281 (holotype: WUK?; isotypes: CDBI (image!), PE!).

Herb (12.7-)57-100(-146) cm high, loosely tufted, shortly rhizomatous; nodes (2-)4-5(-7), separated along the culm. Basal leaf-blades (3-)5.3-10.5(-17.5) cm long $\times(2-) 2.7-$ 4.3(-7.7) mm wide; top culm leaf-sheaths $(8-) 13-20(-25) \mathrm{cm}$ long, glabrous to puberulous or pubescent on the upper part, without, rarely with, cilia on the margins; top culm leaf-blades (1.5-)8.2-20(-34.5) cm long $\times(2.5-) 4-7.2(-11) \mathrm{mm}$ wide. Panicles (5-)11.7-16(-23) cm long $\times(1.6-) 3-5(-10) \mathrm{cm}$ wide, elliptic to oblong in outline, lax to slightly dense; longest basal branches (1-)2-4.5(-10.5) cm long. Pedicels (1.3-)36.3(-10.5) $\mathrm{mm}$ long. Lemmas (4-)5.4-6.8(-7.8) $\mathrm{mm}$ long; awn (3-)6.7-10(-11.7) mm long. Figure 2.

Chromosome Number-2n= 14 (Ono and Tateoka 1953; Tateoka 1978; Frey 1992).

Phenology-Flowering and fruiting from June to October.

Distribution and Habitat-Trisetum sibiricum subsp. sibiricum is present in eastern Europe (Poland and Serbia), and widespread throughout Asia, extending in its southern part to Sikkim, and southern China (Sichuan), and in its eastern to Japan, and Kamchatka Peninsula; 0-4,200 m of elevation; at open steppe or marshy meadows, open and sparse forests, among shrubs. Figure 3.

Specimens Examined-CHINA. Gansu: Xiahe County, near the Labu, $35^{\circ} 2^{\prime} \mathrm{N}, 102^{\circ} 29^{\prime} \mathrm{E}, 25$ Jul 1937, Fu 1386 (PE); Minxian County, Mawu, $35^{\circ} 27^{\prime} \mathrm{N}, 106^{\circ} 33^{\prime} \mathrm{E}, 26$ Jun 1936, Wang 4559 (PE). Hebei: Laiyuan county, $39^{\circ} 20^{\prime} \mathrm{N}, 114^{\circ} 40^{\prime} \mathrm{E}, 13 \mathrm{Jul} 1959$, [illegible] 2096 (HNWP). Heilongjiang: Mishan county, $45^{\circ} 33^{\prime} \mathrm{N}, 131^{\circ} 53^{\prime} \mathrm{E}$, 15 Jul 1980, Lishuxin 3118 (IFP); Khingai Mountains, Saltanovka, $48^{\circ} 45^{\prime} \mathrm{N}, 127^{\circ} 0^{\prime} \mathrm{E}, 16$ Aug 1902, Litvinov 768 (AAH, GH, NY). Inner Mongolia: Bayan Obo, 41 ${ }^{\circ} 46^{\prime} \mathrm{N}, 109^{\circ} 58^{\prime} \mathrm{E}, 24 \mathrm{Jul} 1962$, Meng ning Team 1262 (PE). Jilin: vallis Badaochesa, fluvium Sui-fum, 43ำ $11^{\prime} \mathrm{N}$, $127^{\circ} 27^{\prime} \mathrm{E}, 21$ Jun 1896, Komarov s. $n$. (P). Qinghai: Tongde, Hebei, Gongma, $36^{\circ} 26^{\prime} \mathrm{N}$, $98^{\circ} 9^{\prime} \mathrm{E}, 29 \mathrm{Jul}$ 1990, [illegible] 5226 (HNWP). Shanxi: Ba ji ling, Ma jia zhuang, Ning wu dong zhai, 13 Sep 1953, Shanxi Team 246 (PE); Nigwu, $38^{\circ} 50^{\prime} \mathrm{N}, 112^{\circ} 9^{\prime} \mathrm{E}, 29 \mathrm{Jul}$ 1957, Shanxi Team 1938 (MO, PE); Chiao-ch'eng distr., Pa-shui-kou-shan, $38^{\circ} 30^{\prime} \mathrm{N}, 111^{\circ} 0^{\prime} \mathrm{E}, 24$ Aug 1924, Smith 7173 (BM, PE, S); Ning-wu Hsien, 38 50'N, 112 ${ }^{\circ}{ }^{\prime} \mathrm{E}, 21$ Aug 1929, Tang 1433 (US); Fangshan, $37^{\circ} 53^{\prime} \mathrm{N}, 111^{\circ} 14^{\prime} \mathrm{E}$, 3 Aug 1955, Wenzhong Wang 141 (PE); Guandi mountain, 37 $52^{\prime} \mathrm{N}, 111^{\circ} 32^{\prime} \mathrm{E}, 5$ Jul 1955, Yellow river Team 2552 (PE); Li cai shan, cong town, 9 Sep 1955, Yellow river Team 2614 (PE).

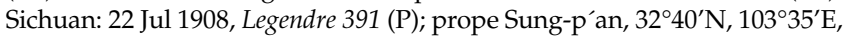
14 Jul 1922, Smith 2772 (BM, PE); San-ch’a-tzü [Sanchazi], 3247'N, $103^{\circ} 16^{\prime} \mathrm{E}, 10$ Aug 1922, Smith 3447 (MO, PE); Tongolo, 30 ${ }^{\circ} 3^{\prime} \mathrm{N}, 101^{\circ} 29^{\prime} \mathrm{E}, 22$ Aug 1894, Soulié s. n. (P); Jinchuan, $27^{\circ} 11^{\prime} \mathrm{N}, 102^{\circ} 17^{\prime} \mathrm{E}, 15 \mathrm{Jun} 1983$, Xiaoen Tian 1089 (PE); Seda, Zhamatang, 32²7’N, 100¹9’E, 7 Sep 1983, Xiaozong

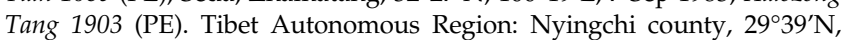

94²2’E, 8 Aug 1983, [illegible] 6249 (PE). Xinjiang: Eastern Tian Shan, left tributary of the Kunges river, $43^{\circ} 8^{\prime} \mathrm{N}, 83^{\circ} 30^{\prime} \mathrm{E}$, 7 Aug 1958, Yunatov 361 (LE); Eastern Tian Shan, Aksu, $50 \mathrm{~km}$ from Kalmal-Kure, $41^{\circ} 11^{\prime} \mathrm{N}, 80^{\circ} 16^{\prime} \mathrm{E}$, 24 Aug 1957, Yunatov 1517 \& al. (LE).

JAPAN. Hokkaido Island: Kunajiri, Veslovsky Peninsula, Golovnino, $43^{\circ} 41^{\prime} \mathrm{N}, 145^{\circ} 32^{\prime} \mathrm{E}, 6$ Aug 1960, Czerniawa s. $n$. (C); côte de Saruru, $44^{\circ} 26^{\prime} \mathrm{N}$,

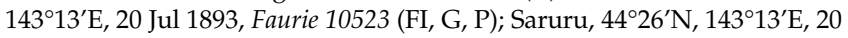
Jul 1893, Faurie 10524 (WU); Ile de Rebunshiri, 44 ${ }^{\circ} 58^{\prime} \mathrm{N}, 147^{\circ} 47^{\prime} \mathrm{E}, 1$ Aug 1892, Faurie 8456 (FI, G); Nemuro prov., Bekkaido-choo, Notsuke-gun,

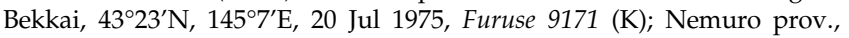
Bekkaido-choo, Notsuke-gun, Okuyukiusu, $43^{\circ} 18^{\prime} \mathrm{N}, 145^{\circ} 12^{\prime} \mathrm{E}, 21 \mathrm{Jul} 1975$, Furuse 9176 (K); Rishiri Island, $45^{\circ} 10^{\prime} \mathrm{N}, 141^{\circ} 14^{\prime} \mathrm{E}, 20$ Jul 1929, Saito s. n. (B).

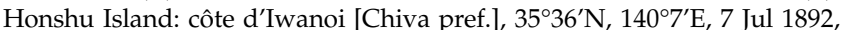
Faurie (P); Kai Prov., Nagano pref., near summit Mt. Kita-dake, Ashi-yasu-son, Naka-koma-gum, $35^{\circ} 40^{\prime} \mathrm{N}, 138^{\circ} 14^{\prime} \mathrm{E}, 31 \mathrm{Jul} 1969$, Furuse 47899 (K); Suruga prov., Shidzuoka pref., near summit from half way Mt. Semmai-dake, $35^{\circ} 25^{\prime} \mathrm{N}, 138^{\circ} 13^{\prime} \mathrm{E}, 28$ Aug 1958, Furuse s. $n$. (AAH, K, S); Suruga prov., Shidzuoka pref., Igawa-mura, Abe-gun, Mt. Shiwomi-dake, $35^{\circ} 34^{\prime} \mathrm{N}$, $138^{\circ} 10^{\prime} \mathrm{E}, 23$ Aug 1957, Furuse s. n. (AAH, S); Shinano prov., Nagano pref., Karuizawa-machi, Minami-karuizawa, 36 21 'N, 138 $35^{\prime} \mathrm{E}, 16$ Jul 1962, Furuse s. n. (AAH, S); Mt. Ibukiyama, $35^{\circ} 25^{\prime} \mathrm{N}, 136^{\circ} 24^{\prime} \mathrm{E}$, 5 Jul 1942, Hashimoto 9721 (US); Tochigi pref., Nikko city, Akanagisan, $36^{\circ} 48^{\prime} \mathrm{N}$, $139^{\circ} 34^{\prime} \mathrm{E}$, Jul 1904, Makino s. n. (AAH); Nagano pref., Kirigamine, $36^{\circ} 6^{\prime} \mathrm{N}$, $138^{\circ} 10^{\prime} \mathrm{E}$, 1 Aug 1935, Ohwi 8305 (US); Yamanashi pref., Kitadake mountain, $35^{\circ} 40^{\prime} \mathrm{N}, 138^{\circ} 14^{\prime} \mathrm{E}, 23$ Aug 1929, Ohwi s. n. (GH, US); Kirigamine, $36^{\circ} 6^{\prime} \mathrm{N}, 138^{\circ} 10^{\prime} \mathrm{E}, 23 \mathrm{Jul} 1952$, Tateoka s. $n$. (B); Shizuoka pref., Shimoda, $34^{\circ} 40^{\prime} \mathrm{N}, 138^{\circ} 57^{\prime} \mathrm{E}$, Wright s. n. (NY, P).

KAZAKHSTAN. Almaty Oblysy: Dzungar Alatau, Northern slope of Mount Chemuldyk, $44^{\circ} 45^{\prime} \mathrm{N}, 79^{\circ} 20^{\prime} \mathrm{E}, 19$ Aug 1948, Goloskokov s. n. (LE); vicinity of of Lepsinsk, Bel-Terek natural landmark, $45^{\circ} 31^{\prime} \mathrm{N}, 80^{\circ} 36^{\prime} \mathrm{E}, 20$ Jun 1928, Lipshits 249 (LE); Kopal uyezd, gorge of the Baskana river, $45^{\circ} 8^{\prime} \mathrm{N}$, 79³'E, 24 Jul 1909, Lipshits 3044 (LE); Dzhungar Alatau, upper reaches of the Chin-bulak river, $43^{\circ} 17^{\prime} \mathrm{N}, 77^{\circ} 9^{\prime} \mathrm{E}, 24$ Aug 1930, Shipchinskiy 529 (LE); Tian Mountains, in montibus Transiliensibus ad fl. Almatinka Minorem,

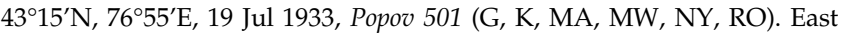
Kazakhstan: ad fl. Ishar and Ulba, 49 57'N, 82³5'E, Bunge 101 (P); Zyryanovsk distr., $49^{\circ} 43^{\prime} \mathrm{N}, 84^{\circ} 16^{\prime} \mathrm{E}$, $24 \mathrm{Jul} 1936$, Temnoev 62 (LE).

KIRGIZSTAN. Bishkek: gorge of the Karagaydy-Bulak river, $42^{\circ} 52^{\prime} \mathrm{N}$, 74³5’E, 20 Jul 1916, Sovetkina 2706 \& Chausova (LE). Issyk-Kul Region: Central Tian-Shan, southern slope of the Kungei-Alatau range, basin of the Cholpan-ata river, [Keshch]-kurgan natural landmark, $42^{\circ} 43^{\prime} \mathrm{N}, 77^{\circ} 25^{\prime} \mathrm{E}, 30$ Jul 1935, Gomolitskiy 716 \& Semenikhina (LE); between the Tyupka and the Karkara rivers, $42^{\circ} 10^{\prime} \mathrm{N}, 78^{\circ} 28^{\prime} \mathrm{E}, 27 \mathrm{Jul} 1950$, Medvedeva 641 \& al. (LE); Karkar River Gorge, $42^{\circ} 0^{\prime} \mathrm{N}, 7^{\circ} 30^{\prime} \mathrm{E}, 21$ Jul 1962, Vintergoller s. n. (FI).

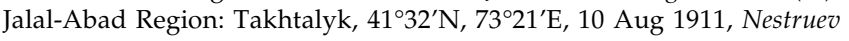
1871 (LE). Naryn Region: North-western slope of Maliy Kalgar mount to the Cholpan-ata river, $41^{\circ} 5^{\prime} \mathrm{N}, 76^{\circ} 45^{\prime} \mathrm{E}, 15 \mathrm{Jul}$ 1936, Semenikhina 183 (LE).

MONGOLIA. Arkhangai: northern slope of Orkhon-Selenga watershed, $47^{\circ} 33^{\prime} \mathrm{N}, 102^{\circ} 49^{\prime} \mathrm{E}$, 21 Oct 931, Desiatkin 502 (LE); Khan-Kokshun mount, the Baga-Targyl gorge, $47^{\circ} 30^{\prime} \mathrm{N}, 100^{\circ} 0^{\prime} \mathrm{E}, 17$ Aug 1926, Gusev 256 (LE). Bulgan: a vico Uňt, $49^{\circ} 8^{\prime} \mathrm{N}, 102^{\circ} 49^{\prime} \mathrm{E}, 11$ Aug 1965, Deyl 2917 \& Sojak (PR). Khentii: Montes Kentei, ad fontes fluviorum Tola, Mensa et Iro, $48^{\circ} 53^{\prime} \mathrm{N}, 109^{\circ} 10^{\prime} \mathrm{E}, 13$ Aug 1929, Ikonnikov-Galitzky 3426 \& IkonnikovGalitzky (AAH); Khentei range,Tsenkher gol opposite to Tsenkheriin dugang, 47 $45^{\prime} \mathrm{N}, 109^{\circ} 3^{\prime} \mathrm{E}$, 22 Jul 1949, Yunatov 15678 (LE). Khövsgöl: vicinity of Kosogol lake, near Khatkhyl, 50 $26^{\prime} \mathrm{N}, 100^{\circ} 9^{\prime} \mathrm{E}, 10$ Aug 1925, Burdukova s. n. (LE); a vico Tarialan [Tarjalang], inter oppida Bulgan et Muren, $49^{\circ} 37^{\prime} \mathrm{N}, 102^{\circ} 3^{\prime} \mathrm{E}, 13$ Aug 1965, Deyl 3268 \& Sojak (PR); lacus Chubsugul versus septentrionem a vico Chadchal, $51^{\circ} 6^{\prime} \mathrm{N}, 100^{\circ} 30^{\prime} \mathrm{E}, 16$ Aug 1965, Deyl s. n. \& Sojak (PR). Övörkhangai: Altai Gobicus, Baga-Bogd-úl

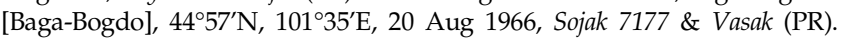
Selenge: Kentei mountains, on the way to Dulan Khan daban, $49^{\circ} 57^{\prime} \mathrm{N}$, $106^{\circ} 12^{\prime} \mathrm{E}$, Ikonnikov-Galitzky 3426 \& Ikonnikov-Galitzky (LE). Töv: montes Chentej, ad viam ex urbe Ulánbátar ad riv. Terelz, $48^{\circ} 10^{\prime} \mathrm{N}, 107^{\circ} 12^{\prime} \mathrm{E}, 6$ Sep 1966, Vasak 9247 (PR). Ulaanbaatar: in valle Nucht ad declivia montium Bogdúl, 4754'N, 10652’E, 24 Jul 1965, Deyl 344 \& Soják (PR); ad ripam dextram rivi Tola apud vicum Songino $(25 \mathrm{~km}$ austro-occid. ab opp. Ulan-Bator), 47 $54^{\prime} \mathrm{N}, 106^{\circ} 52^{\prime} \mathrm{E}$, 31 Jul 1965, Deyl s. n. \& Soják (PR); on Mount Bogdo-ula, $47^{\circ} 49^{\prime} \mathrm{N}, 107^{\circ} 0^{\prime} \mathrm{E}$, 27 Jul 1927, Ikonnikov-Galitzky 119 (LE); Ulan Bator, $47^{\circ} 54^{\prime} \mathrm{N}$, $106^{\circ} 52^{\prime} \mathrm{E}, 1$ Aug 1961, Soják s. n. (PR).

POLAND. Kuyavia-Pomerania: $80 \mathrm{~km}$ NW Torun (Thorn), zwischen Kesowo und Stawecin bei Obrowo nach Süden, 53 $3^{\circ} 33^{\prime} \mathrm{N}, 17^{\circ} 42^{\prime} \mathrm{E}, 13$ Jun 1991, Angerer s. n. (M); Podlaskie: Bialystok province, Białowieża National Park, meadows on the Narewka river, $52^{\circ} 42^{\prime} \mathrm{N}, 23^{\circ} 52^{\prime} \mathrm{E}, 7 \mathrm{Jul} 1982$, Frey s. n. (L).

RUSSIA. Altai Krai: Biysk okrug, system of the Katun river, Chuiskiy tract, Shabolin vicinity, $52^{\circ} 32^{\prime} \mathrm{N}, 85^{\circ} 12^{\prime} \mathrm{E}, 6$ Jul 1904, Klements 275 (LE); 


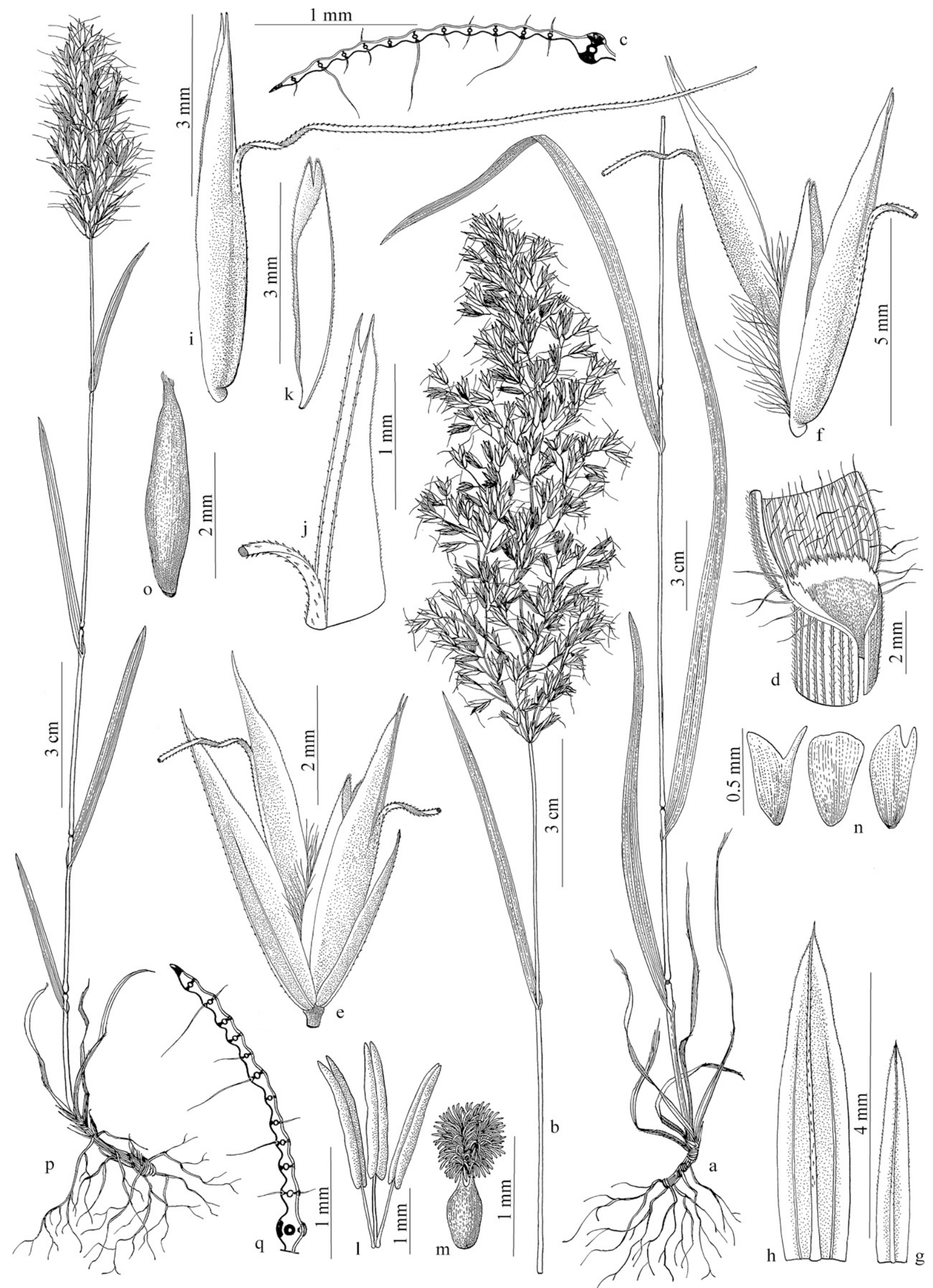

Fig. 2. Trisetum sibiricum subsp. sibiricum. a. Basal habit. b. Inflorescence. c. Transverse section of leaf-blade. d. Sheath, ligule, and portion of the blade. e. Spikelet. f. Florets. g. Lower glume, dorsal view. h. Upper glume, dorsal view. i. Floret. j. Lemma, upper part, lateral view. k. Palea, lateral view. 1. Stamens. m. Pistil. n. Lodicules. o. Caryopsis. Trisetum sibiricum subsp. litorale. p. Habit. q. Transverse section of leaf-blade. (Sojak s. n., PR-807346, a-b; Popov 501, G-442513, c; Sojak s. n., PR-807345, d-n; Deyl E Sojak 4090, PR-807035, o; Koroleva E Petrovsky 5703, M-223301, p; Solstad E Elven 04_0266A, O, q). 


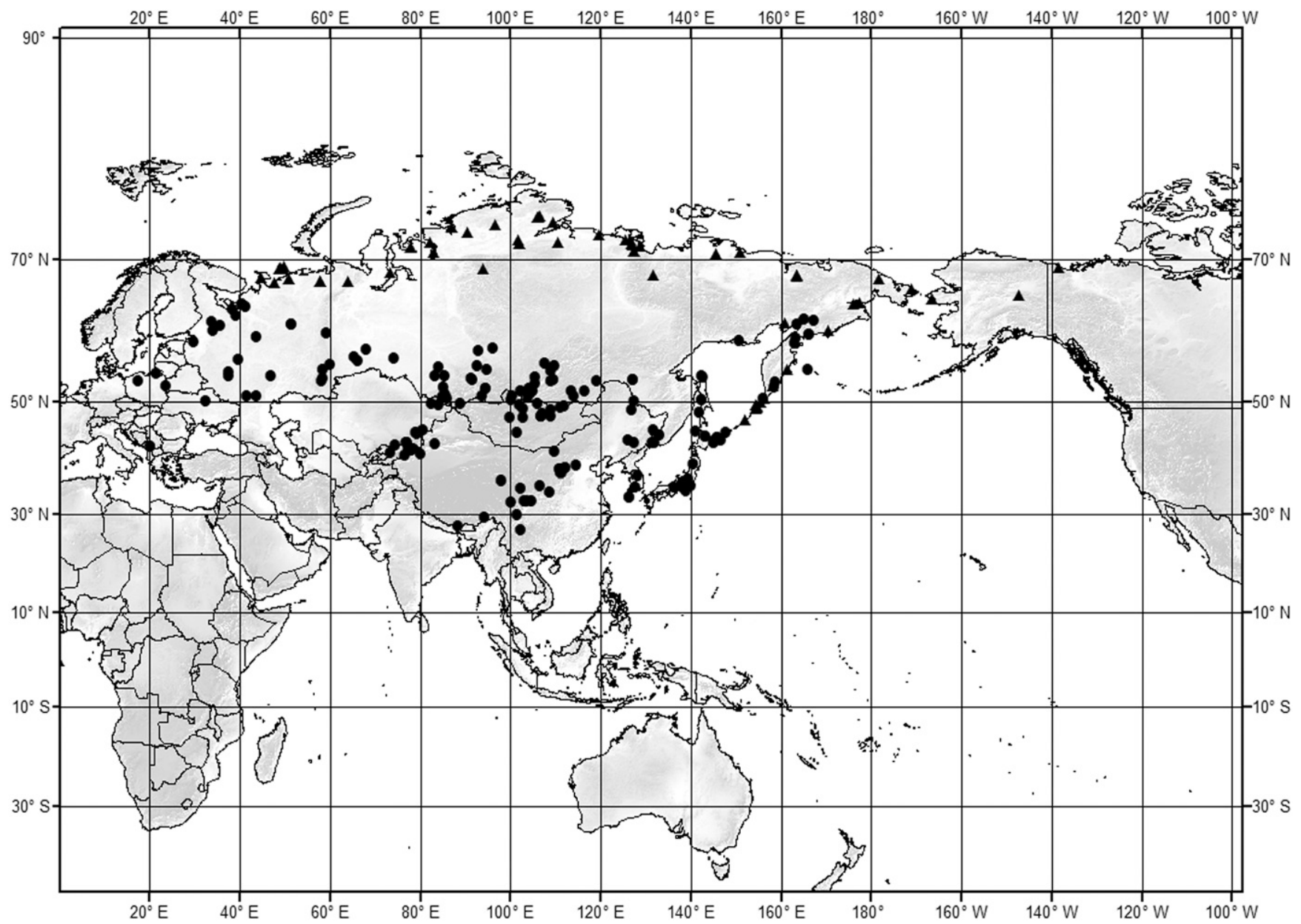

FIG. 3. Distribution of Trisetum sibiricum subsp. sibiricum (dots) and T. sibiricum subsp. litorale (triangles).

Shebalino area, $3 \mathrm{~km} \mathrm{~W}$ of Cherga, 51 $33^{\circ} \mathrm{N}, 85^{\circ} 31^{\prime} \mathrm{E}, 16$ Aug 1985, Alanko 52917 \& al. (H); Gorno-altaisk, west side of Yabogan Pass, 50 ${ }^{\circ} 54^{\prime} \mathrm{N}, 8^{\circ} 4^{\prime} \mathrm{E}$, 31 Aug 1978, Elias 4847 \& al. (NY); Ondugay distr., Eloman, Ailiagushi river valley, $50^{\circ} 45^{\prime} \mathrm{N}, 86^{\circ} 9^{\prime} \mathrm{E}$, 14 Oct 1936 , Kalinina s. $n$. \& al. (LE); Shebalinskiy Rayon, Sarlyk river valley, $10 \mathrm{~km}$ ESE Topuchaya, $51^{\circ} 6^{\prime} \mathrm{N}$, $85^{\circ} 39^{\prime} \mathrm{E}$, $31 \mathrm{Jul}$ 2008, Martins 2301 (JE); in valle fl. Taschte, in fl. Czebdar influent, 11 Aug 1927, Schischkin s. n. (NY); in jugum Sajlügem, fl. Bugusum, $49^{\circ} 55^{\prime} \mathrm{N}$, 8859'E, 18 Aug 1931, Schischkin s. n. \& Chilikina (NY); Ausläufer des Seminskij Rückens, etwa $50 \mathrm{~km} \mathrm{~S}$ Cherga, $51^{\circ} 10^{\prime} \mathrm{N}, 8^{\circ} 34^{\prime} \mathrm{E}, 25 \mathrm{Jul} 1996$, Sukopp 1658 (B). Amur: vicinity of the Zeia-Pristan, Pikan meteorological station, $53^{\circ} 42^{\prime} \mathrm{N}, 127^{\circ} 20^{\prime} \mathrm{E}, 14 \mathrm{Jul} 1910$, Evstifeeva 360 (LE); Amur-Zeia plateau, $1.5 \mathrm{~km}$ south-west of the mouth of the Belyi stream, $53^{\circ} 44^{\prime} \mathrm{N}$, $127^{\circ} 15^{\prime} \mathrm{E}, 5 \mathrm{Jul} 1958$, Isachenko 219 \& al. (LE); Blagoveshchensk, 50¹7'N, $127^{\circ} 24^{\prime} \mathrm{E}$, Jul 1899, Karo s. n. (G). Arkhangelsk: Tschurkina, $64^{\circ} 32^{\prime} \mathrm{N}$, $40^{\circ} 32^{\prime} \mathrm{E}$, 18 Aug 1891, Kihlman s. n. (H); Jarnema, $62^{\circ} 57^{\prime} \mathrm{N}, 3^{\circ} 21^{\prime} \mathrm{E}, 17 \mathrm{Aug}$ 1899 , Liro s. n. \& Cajander (H); Boljsjezemelskaja, $64^{\circ} 32^{\prime} \mathrm{N}, 40^{\circ} 32^{\prime} \mathrm{E}$, $24 \mathrm{Jul}$ 1964, Rebristaja 553 \& Gokorevskich (GB); left side between the Khudaya Sarova and Verkhnyaya rivers, $64^{\circ} 16^{\prime} \mathrm{N}, 41^{\circ} 20^{\prime} \mathrm{E}, 25 \mathrm{Jul} 1928$, Zubkov s. $n$. (LE). Bashkortostan Republic: Mesyagutov, Lakly, Lasyn-tash mount, $55^{\circ} 11^{\prime} \mathrm{N}, 58^{\circ} 32^{\prime}$ E, 29 Jun 1928, Noskov 291 \& al. (LE); Beloretsk distr., Bekhta range, $53^{\circ} 58^{\prime} \mathrm{N}, 58^{\circ} 24^{\prime} \mathrm{E}, 18$ Aug 1940 , Sokolova s. $n$. (LE); Southern Ural, Bashkir State Nature Reserve, Bolshoy Shatak mount, 53 $33^{\prime} \mathrm{N}, 58^{\circ} 12^{\prime} \mathrm{E}, 11$ Aug 1946, Selivanova-Gorodkova 1521 (LE). Buryatia Republic: Lake Baikal,

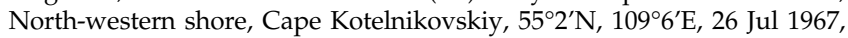
Egorova s. n. \& Siplivinskiy (LE); Barguzin distr., basin of the Vitim, vicinity of the Polivtsev winter hut, $53^{\circ} 37^{\prime} \mathrm{N}, 109^{\circ} 38^{\prime} \mathrm{E}, 1908$, Iarygin 147 (LE); Barguzin distr., basin of the Vitim river, the vicinity of Endonginskoe winter hut, $53^{\circ} 37^{\prime} \mathrm{N}, 109^{\circ} 38^{\prime} \mathrm{E}, 1908$, Khutov 130 (LE); Selenginsk uyezd, weather station of Verkhnyaya Mishikha, 50 $49^{\prime}$ N , $103^{\circ} 53^{\prime} \mathrm{E}, 8$ Aug 1903, Litvinov 898 (LE); montes Sajany, mont. Tunkinskie golcy supra pag. Arsan, $51^{\circ} 54^{\prime} \mathrm{N}, 102^{\circ} 25^{\prime} \mathrm{E}, 20$ Jul 1961, Soják s. n. (PR); Barguzin distr., UstBarguzin, near Makarinina, $53^{\circ} 24^{\prime} \mathrm{N}, 109^{\circ} 1^{\prime} \mathrm{E}, 13$ Jul 1916, Larin s. $n$. \& Kanerskiy (LE); selo of Verkhne-Angarskoe, 5550’N, 10958'E, 30 Jun 1912,
Shipchinskiy 145 (LE). Chelyabinsk: Verkhniy Ufalei, above the Ufalei river, $56^{\circ} 3^{\prime} \mathrm{N}, 60^{\circ} 14^{\prime} \mathrm{E}, 27$ Jun 1939, Igoshina s. n. (LE). Irkutsk: Baikal station (Cape

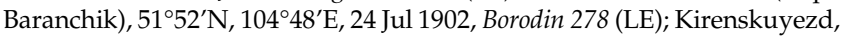
Okunaika river, near Dushekanskaia path, 56 ${ }^{\circ} 6^{\prime} \mathrm{N}, 107^{\circ} 39^{\prime} \mathrm{E}, 14 \mathrm{Jul} 1914$, Drobov 384 (LE); Cherski Peak, confluence of Angara River with Lake Baikal, ca. 2 km E of Listvyanka, 51 ${ }^{\circ} 51^{\prime} \mathrm{N}, 104^{\circ} 54^{\prime} \mathrm{E}, 8 \mathrm{Jul} 1979$, Iltis 561 \& al. (NY); Polovinca, $53^{\circ} 7^{\prime} \mathrm{N}, 105^{\circ} 39^{\prime} \mathrm{E}$, Aug 1902, Lönnbohm s. n. (H); Lake Baikal, near the mouth of the Snezhnaya river, $5^{\circ} 28^{\prime} \mathrm{N}, 104^{\circ} 37^{\prime} \mathrm{E}, 26 \mathrm{Jul}$ 1915, Sukachev 2577 \& Poplavskaya (LE); Kulenga river Valley, 54 ${ }^{\circ} 5^{\prime} \mathrm{N}$, $105^{\circ} 35^{\prime}$ E, 2 Jul 1908, Tomin 153 (LE). Kamchatka Krai: Olyutorskiy distr., Korfa Bay, Kultushnoe, $60^{\circ} 28^{\prime} \mathrm{N}, 166^{\circ} 17^{\prime} \mathrm{E}, 2$ Aug 1960, Vasilkova s. n. \& al. (LE); Yelizovsky distr., $50 \mathrm{~km} \mathrm{~S}$ from Petropavlovsk-Kamchatsky, Zhirovaya bay, $5^{\circ} 36^{\prime} \mathrm{N}$, $158^{\circ} 24^{\prime} \mathrm{E}, 7 \mathrm{Jul} 2010$, Vislobokov s. n. (MW); Karaginsky distr., shore of Penzhinskaia Guba, 2-3 km NE of Cape Kingi, $61^{\circ} 48^{\prime} \mathrm{N}, 163^{\circ} 33^{\prime} \mathrm{E}$, Aug 1989, Dorofeev 73 (LE); Penzhinskiy distr., Penzhina river, 12 km

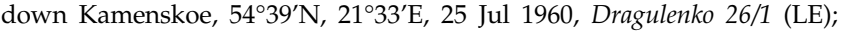
Koryakskaya volcano, $53^{\circ} 19^{\prime} \mathrm{N}, 158^{\circ} 42^{\prime} \mathrm{E}$, 14 Aug 1928, Eyerdam s. n. (F); basin of the Penzhina river, upper reaches of the Palmatkina river, $62^{\circ} 24^{\prime} \mathrm{N}$, $167^{\circ} 13^{\prime} \mathrm{E}$, 14 Aug 1932, Gorodkov s. n. \& Tikhomirov (LE); Toporkov Island, Sarannaja Bay, $55^{\circ} 12^{\prime} \mathrm{N}, 165^{\circ} 56^{\prime} \mathrm{E}, 8$ Aug 1920, Hultén 798 (GB); Kamchatka river, left side of the Belaya river, $15 \mathrm{~km}$ away from the mouth, $59^{\circ} 49^{\prime} \mathrm{N}$, $163^{\circ} 23^{\prime} \mathrm{E}, 14$ Aug 1930, Karev 669 (LE); Karaginsky distr., near Ossora, $59^{\circ} 14^{\prime} \mathrm{N}, 163^{\circ} 4^{\prime} \mathrm{E}, 6$ Aug 1976, Kharkevich s. n. \& Buch (GH); Penzhinskiy distr., Penzhina river, $25 \mathrm{~km}$ away from the mouth, $62^{\circ} 28^{\prime} \mathrm{N}, 165^{\circ} 6^{\prime} \mathrm{E}, 14$ Aug 1960, Kuldiusherskiy 110/2 (LE); Olyutorskiy distr., Korfa Bay, vicinity of the poselok of Kultushnoe, $60^{\circ} 28^{\prime} \mathrm{N}, 166^{\circ} 17^{\prime} \mathrm{E}, 2$ Aug 1960, Vasilkova s. $n$. \& al. (LE). Karelia Republic: Porog, $63^{\circ} 49^{\prime} \mathrm{N}, 38^{\circ} 28^{\prime} \mathrm{E}, 17 \mathrm{Jul} 1875$, Elfuing s. n. (H); Munjärvi, Lettonütylla, $62^{\circ} 14^{\prime} \mathrm{N}, 33^{\circ} 49^{\prime} \mathrm{E}, 3$ Jul 1942, Tuomikoshi s. $n$. (H). Komi Republic: systema fl. Vyczegda, distr. Kortkeros, prope pag. Konscha, 61 ${ }^{\circ} 48^{\prime} \mathrm{N}, 51^{\circ} 34^{\prime} \mathrm{E}, 26 \mathrm{Jul}$ 1909, Andreev 4906a (C, G, JE, M); 28 Jun 1909, Andreev 4906b (C, F, G, JE, M, MO, NY). Krasnoyarsk Krai: Yeniseysky distr., Manzia river, $58^{\circ} 29^{\prime} \mathrm{N}, 9^{\circ} 15^{\prime} \mathrm{E}, 12$ Jul 1908, Blagoveshchenskiy 532 (LE); Minusinsk distr., Potroshilovo, Enisei river, 
$53^{\circ} 55^{\prime} \mathrm{N}, 91^{\circ} 30^{\prime} \mathrm{E}, 9 \mathrm{Jul} 1931$, Iliin 28 \& Ovchinnikov (LE); near Kargino, $58^{\circ} 0^{\prime} \mathrm{N}$, $92^{\circ} 58^{\prime}$ E, 19 Jul 1914, Kuznetsov 78 (LE); Minusinsk distr., between the Gal'zey river and mount of Izyk Erbinskiy, 55 $43^{\prime} \mathrm{N}, 9^{\circ} 49^{\prime} \mathrm{E}, 28 \mathrm{Jul} 1910$, Smirnov 542 (LE). Kuril Islands: Kunashir Island, $44^{\circ} 8^{\prime} \mathrm{N}, 145^{\circ} 52^{\prime} \mathrm{E}, 15$ Aug 1892, Faurie 8504 (P); Paramushir, ca. $3 \mathrm{~km}$ south of Severo-Kurilsk, 50 $0^{\circ} 37^{\prime} \mathrm{N}, 156^{\circ} 7^{\prime} \mathrm{E}, 1$

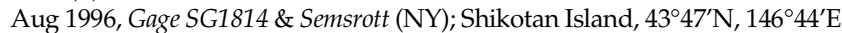
30 Jul 1931, Ohwi 378 (US); Kunashir Island, Furukamapp, 44 $8^{\prime} \mathrm{N}, 145^{\circ} 52^{\prime} \mathrm{E}$, 12 Aug 1923, Ohwi 5261 (US). Leningrad: Podporozh'e distr., Munduksa, $60^{\circ} 54^{\prime} \mathrm{N}, 34^{\circ} 10^{\prime} \mathrm{E}$, Jul 1898, Cajander s. n. \& Lindroth $(\mathrm{H}) ; 19.5 \mathrm{~km}$ of poselok of

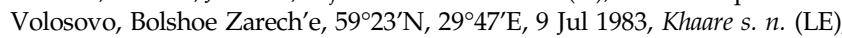
Lake Onega, S of Svir River, Juksovo, 61 ${ }^{\circ} 41^{\prime} \mathrm{N}, 35^{\circ} 39^{\prime} \mathrm{E}$, 1942, Sarvela s. n. (H). Lipetsk: Donskoye distr., Vodopyanovo, Galich'ya Gora Reserve, $51^{\circ} 2^{\prime} \mathrm{N}$,

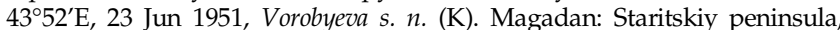
Shelikhov Gulf, $59^{\circ} 33^{\prime} \mathrm{N}, 150^{\circ} 47^{\prime} \mathrm{E}, 1$ Sep 1964 , Iurtsev s. $n$. (LE). Moscow: Lushki ad Occam, Reservatum publicum, flum. Tadenka, 54 $53^{\prime} \mathrm{N}, 37^{\circ} 35^{\prime} \mathrm{E}, 1$ Aug 1963, Smirnow 49 (B, F, G, JE, L, P, S, US). Novosibirsk: ca. $7 \mathrm{~km} \mathrm{~S}$ of village Yevsino, $54^{\circ} 20^{\prime} \mathrm{N}, 83^{\circ} 18^{\prime} \mathrm{E}, 27 \mathrm{Jul} 1979$, Iltis 1321 \& al. (NY, S); $3 \mathrm{~km}$

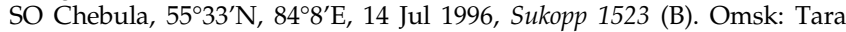
distr., Ciany, 56 50 53’N, 74²2'E, 12 Jul 1922, Seludjakova s. n. (PR). Primorsky Krai: Imansk distr., E of Evgenievka station, Odarka river (basin of Khanka lake), $44^{\circ} 41^{\prime} \mathrm{N}$, $132^{\circ} 48^{\prime} \mathrm{E}$, 17 Jun 1911, Cherskiy 86 (LE); Mongugai river, Ovchinnikovo village, $43^{\circ} 14^{\prime} \mathrm{N}, 1^{\circ} 1^{\circ} 23^{\prime} \mathrm{E}$, 21 Jun 1913, Diukina 539 (LE) Muravyev Amurskiy peninsula, Okeanskaya station, Sadgorod, $43^{\circ} 15^{\prime} \mathrm{N}$, $132^{\circ} 2^{\prime} \mathrm{E}$, 7 Jul 1929, Transhel 153 (LE); $4 \mathrm{~km}$ left of the road from Khvalynka to Konstantinovka, $44^{\circ} 40^{\prime} \mathrm{N}$, $132^{\circ} 59^{\prime} \mathrm{E}$, 4 Jul 1929, Zhirov 223 (LE). Sakhalin: Vladimirof, $50^{\circ} 33^{\prime} \mathrm{N}, 142^{\circ} 36^{\prime} \mathrm{E}$, 30 Jul 1908, Faurie 804 (G, P); Soriofka, $50^{\circ} 33^{\prime} \mathrm{N}$, 142 $36^{\prime} \mathrm{E}$, Aug 1908, Faurie 805 (G, P); $60 \mathrm{~km} \mathrm{NW}$ of Okha, at "Judith Bog", mouth of Antonovka River, Pomr Bay, 54 ${ }^{\circ} 1^{\prime} \mathrm{N}, 142^{\circ} 43^{\prime} \mathrm{E}, 13$ Aug 2003, Legler 1246 (NY); W coast 5 km N of Krasnogorsk, S of Lake Aynskoye, $48^{\circ} 27^{\prime} \mathrm{N}, 142^{\circ} 3^{\prime} \mathrm{E}$, 21 Jul 2003, Legler 773 (NY); Okha distr.

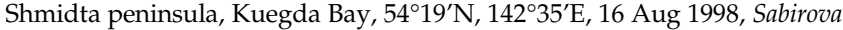
s. n. \& Sabirov (LE). Sverdlovsk: Elovskiy Ural range, $60^{\circ} 35^{\prime} \mathrm{N}, 59^{\circ} 24^{\prime} \mathrm{E}, 24$ Aug 1940, Tikhomirov 73 (LE). Tomsk: Kuznetsk distr., Salair Ridge, between Gavrilovskiy factory and Biryulinskie vysoty, $54^{\circ} 15^{\prime} \mathrm{N}, 85^{\circ} 30^{\prime} \mathrm{E}, 30$ Jun 1916, Utkin s. n. (LE). Tula: pr. Melechowska, $54^{\circ} 11^{\prime} \mathrm{N}, 37^{\circ} 37^{\prime} \mathrm{E}, 7 \mathrm{Jul}$ 1899, Zinger 796 (G, MW). Tuva Republic: Kurtushibinskiy range, upper

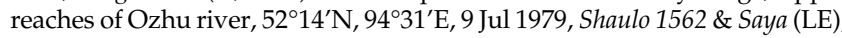
10 Jul 1979, Shaulo 1561 \& Belskaya (LE); northern slope of Tannu-Olarange, Khovu-Aksy, $51^{\circ} 7^{\prime} \mathrm{N}, 93^{\circ} 42^{\prime} \mathrm{E}, 22 \mathrm{Jul} 1973$, Khanmimchun 2207 \& Amelchenko (LE). Tyumen: Mire behind the village of Vorogushina, north of Tobolsk, $58^{\circ} 11^{\prime} \mathrm{N}, 68^{\circ} 15^{\prime} \mathrm{E}$, 2 Jun 1910, Mameev 675 (K, LE, US); Yalutorovsk distr., $56^{\circ} 39^{\prime} \mathrm{N}, 66^{\circ} 18^{\prime} \mathrm{E}$, 14 Jun 1912, Svitich s. n. (LE). Ulyanovsk: Karsunskiy

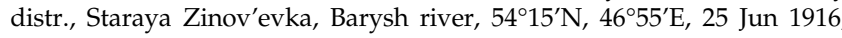
Shennikov s. n. (LE). Vladimir: Yriev-Polskiy distr., $5 \mathrm{~km} \mathrm{S-SE} \mathrm{of} \mathrm{Shorodoga,}$ Nerl' river, 56 $6^{\circ} 6^{\prime} \mathrm{N}, 39^{\circ} 51^{\prime} \mathrm{E}$, $11 \mathrm{Jul} 2007$, Seregin 3135 (MW). Vologda: road

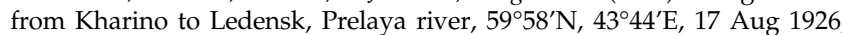
Korchagin 169 \& Gaze (LE).Voronezh: Khoper Nature Reserve, Bogdanovskiy forest, $12-14 \mathrm{~km}$ NE Novokhopersk, $51^{\circ} 5^{\prime} \mathrm{N}$, $41^{\circ} 37^{\prime} \mathrm{E}$, 9 Jun 1980 Tsvelev 266 (LE). Zabaykalsky Krai: Nerchinsko-Zavodsky distr., between Davenda and Kudecha rivers, 53 $33^{\prime} \mathrm{N}, 119^{\circ} 19^{\prime} \mathrm{E}, 19$ Jul 1909, Blagoveshchenskiy 1248 \& Poplavskaia (LE); Aginskoe, Anzhi river, 55ำ $15^{\prime} \mathrm{N}, 94^{\circ} 54^{\prime} \mathrm{E}$, 16 Jul 1949, Fedorov s. n. \& al. (LE); Kyrinskiy distr., 17 km S of Kyra, $49^{\circ} 34^{\prime} \mathrm{N}, 111^{\circ} 58^{\prime} \mathrm{E}, 12$ Aug 2005, Golovina 310 (LE); Nertschinsk, 51 $59^{\circ} \mathrm{N}$, $116^{\circ} 35^{\prime} \mathrm{E}, 1889$, Karo 270 (G, GOET, WU); prope pag. Taptanai, $51^{\circ} 5^{\prime} \mathrm{N}$, $114^{\circ} 15^{\prime} \mathrm{E}, 12 \mathrm{Jul} 1931$, Krylov s. n. (NY); Chitinskiy Rayon, $52^{\circ} 0^{\prime} \mathrm{N}, 113^{\circ} 30^{\prime} \mathrm{E}$ 14 Jul 1913, Poplawska 1743 (K); Akshinsk distr., mountains along Bukukun river, near poselok of Bukukunskiy, $49^{\circ} 19^{\prime} \mathrm{N}, 111^{\circ} 15^{\prime} \mathrm{E}, 21 \mathrm{Jul} 1913$, Smirnov 70 (LE).

SERBIA. Vlasina lake, $42^{\circ} 42^{\prime} \mathrm{N}, 2^{\circ} 22^{\prime} \mathrm{E}, 1881$, Pančić s. $n$. (W)

SOUTH KOREA. Gangwon: Ouen-San mountain, $37^{\circ} 30^{\prime} \mathrm{N}, 128^{\circ} 15^{\prime} \mathrm{E}$, Jul

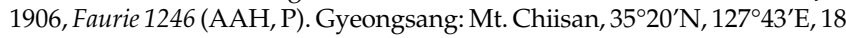
Jul 1942, Nakashima s. n. (US). Jeju: Quelpart Island, Hallasan crater, $33^{\circ} 24^{\prime} \mathrm{N}, 126^{\circ} 32^{\prime} \mathrm{E}, 13$ Aug 1908, Taquet 1891 (G, K, NY); Jul 1909, Taquet 3423 (AAH, NY).

UKRAINE. Poltava: terrace above the Udoy river near Kalinoviy most, $50^{\circ} 16^{\prime} \mathrm{N}, 32^{\circ} 28^{\prime} \mathrm{E}, 29$ Jun 1927, Poretskiy 298 (LE).

b. subsp. LITORAlE Rupr. ex Roshev., Izv. Glavn. Bot. Sada R. S. F.S. R. 21: 90. 1922. Trisetum sibiricum var. litorale (Rupr. ex Roshev.) Rupr. ex Roshev. in V.L. Komarov (ed.), Fl. URSS 2: 254. 1934. Trisetum litorale (Rupr. ex Roshev.) Khokhr. Botan. Zhurn. 63 (3): 395. 1978, nom. illeg., non Phil. 1858. Trisetaria sibirica subsp. litoralis (Rupr. ex Roshev.) Banfi \& Soldano, Atti Soc. Ital. Sci. Nat. Mus. Civico Storia Nat. Milano 135: 385. 1996. Trisetum ruprechtianum Tzvelev,
Novosti Sist. Vyssh. Rast. 42: 89. 2011.-TYPE: RUSSIA. Kanin Peninsula, 67 $27^{\prime} \mathrm{N}$ 47 $32^{\prime} \mathrm{E}, 13-14 \mathrm{Aug}$, F. J. I. Ruprecht s. n. (lectotype, designated by Tzvelev (1976: 262): LE!).

Herb (12.7-)23.8-37.7(-58.4) cm high, not tufted, shortly rhizomatous or with stoloniferous rhizomes; nodes 3-4(-5), separated or sometimes concentrated in lower part of the culm, enclosed or not by the sheaths. Basal leaf-blades (1.2-)2$4.3(-10.5) \mathrm{cm}$ long $\times(2-) 2.3-3.4(-4.8) \mathrm{mm}$ wide; top culm leafsheaths (4.2-)6-9(-13.7) cm long, glabrous, rarely with hairs on the nerves and upper part, without cilia on the margins; top culm leaf-blades (3.2-)4.5-7.5(-13.7) cm long $\times(2.7-) 3.6-$ 4.4(-7) mm wide. Panicles (3.3-)5-7.4(-9) cm long $\times(1.2-)$ $1.8-2.6(-3.8) \mathrm{cm}$ wide, ovate, sometimes elliptic or oblong in outline, dense; longest basal branches (0.6-)1-2.3(-2.8) cm long. Pedicels (0.5-)1.5-2.8(-4) mm long. Lemmas (3.8-)4.75.7(-6.2) mm long; awn (4-)5.5-7(-8.4) mm long. Figure 2.

Chromosome Number-2 $n=14$ (Zhukova 1967).

Phenology-Flowering and fruiting from July to September.

Distribution and Habitat-This subspecies is distributed along the Russian coast, from Kanin Peninsula to Kamchatka Peninsula and Kuril Archipelago, extending to Alaska and Arctic coast of Yukon territory in Canada; 0-900 m of elevation; in meadows, sandy or rocky riversides, and also in tundra. Figure 3.

Specimens Examined-CANADA. Yukon: Arctic Coast west of Mackenzie River delta, between King Point and Kay Point, $69^{\circ} 12^{\prime} \mathrm{N}$, $138^{\circ} 30^{\prime}$ W, Jul 1934, Porsild 7122 (S).

RUSSIA. Arkhangelsk: Bolshezemelskaya tundra, Vangurei range

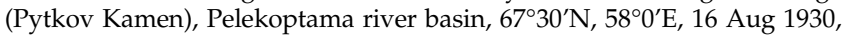
Andreev 692 \& Savkina (LE); Bolshezemelskaya tundra, Vangurei range (Pytkov Kamen), Khylchou river basin, 67 $30^{\prime} \mathrm{N}, 58^{\circ} 0^{\prime} \mathrm{E}, 28$ Aug 1930, Andreev 898 \& Savkina (LE); ad ostia fl. Lena, Bulum, Bach-Ufer, 31 Aug 1901, Cajander 2074 (H); Bolshezemelskaya tundra, right side ot the SabreiYaga, $67^{\circ} 30^{\prime} \mathrm{N}, 58^{\circ} 0^{\prime} \mathrm{E}, 3$ Aug 1938, Kuznetsov s. n. (LE); Pai-Khoi, Mount

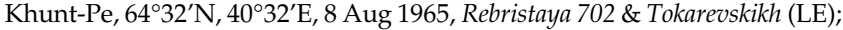
Bolshezemelskaya tundra, $2 \mathrm{~km}$ west of the Iunku-Shor river, $67^{\circ} 30^{\prime} \mathrm{N}$, $58^{\circ} 0^{\prime} \mathrm{E}, 8$ Aug 1938, Zubkova s. n. (LE). Chukotka Autonomous Okrug: Olenek river, $72^{\circ} 58^{\prime} \mathrm{N}, 119^{\circ} 48^{\prime} \mathrm{E}, 27$ Aug 1875, Czekanowski s. n. (LE); prope pagum Kustur, in valle fl. Bytantaj, $68^{\circ} 10^{\prime} \mathrm{N}, 131^{\circ} 40^{\prime} \mathrm{E}, 4 \mathrm{Aug}$ 1959, Jurtzev s. n. (C); Anyui Highlands, Pogynden River, $68^{\circ} 12^{\prime} \mathrm{N}$, $163^{\circ} 32^{\prime} \mathrm{E}, 21 \mathrm{Jul} 1976$, Petrovskyi s. n. \& Koroleva (K); near the mouth river Chegitun, $66^{\circ} 33^{\prime} \mathrm{N}, 171^{\circ} 4^{\prime} \mathrm{W}, 7$ Aug 1971, Sekretareva s. n. \& al. (BM); Anadyr Krai, the basin of the Belaya river, left tributary of the Anadyr, near the mouth of Belaya river, $64^{\circ} 37^{\prime} \mathrm{N}, 176^{\circ} 14^{\prime} \mathrm{E}, 26 \mathrm{Jul} 1929$, Sochava s. n. (LE); Chukchi National Okrug, Anadyr distr., southern parth of the Ust-belskie mountains, $64^{\circ} 44^{\prime} \mathrm{N}, 177^{\circ} 30^{\prime} \mathrm{E}, 14$ Aug 1977, Vasilieva s. $n$. (LE); right side of the Malyi Aniuy river, the middle reaches of the Machvaam river, $68^{\circ} 12^{\prime} \mathrm{N}, 163^{\circ} 32^{\prime} \mathrm{E}, 12 \mathrm{Jul} 1952$, Vikulova s. $n$. (LE); the middle reaches of the Ekiatap river (left side), the Pastbishchnaya river, $67^{\circ} 44^{\prime} \mathrm{N}, 178^{\circ} 18^{\prime} \mathrm{W}, 12$ Aug 1973, Zubakova s. n. \& Razzhivin (LE). Kamchatka Krai: Olyutorskiy distr., $61^{\circ} 0^{\prime} \mathrm{N}, 170^{\circ} 30^{\prime} \mathrm{E}, 27 \mathrm{Jul} 1974$, Kharkevich s. n. \& Buch (H, MO, NY); Olyutorskiy distr., Vyerkhoutourov, $55^{\circ} 16^{\prime} \mathrm{N}, 161^{\circ} 31^{\prime} \mathrm{E}, 25 \mathrm{Jul} 1975$, Kharkevich s. n. \& Buch (K, MO, MW); Yelizovsky distr., $50 \mathrm{~km}$ south from Petropavlovsk-Kamchatsky, Vilyuchinskaya bay, $52^{\circ} 39^{\prime} \mathrm{N}, 158^{\circ} 23^{\prime} \mathrm{E}, 15 \mathrm{Jul} 2010$, Vislobokov s. $n$. (MW). Krasnoyarsk: Taimyr Lake, Bikada-Nguoma river, $74^{\circ} 50^{\prime} \mathrm{N}$, $106^{\circ} 30^{\prime} \mathrm{E}, 18$ Aug 1974, [unknown] 57 (LE); the basin of the Kheta river, the Khatanga river, right side of the Khatanga river near the mouth of the Zhdanikha river, $71^{\circ} 54^{\prime} \mathrm{N}, 102^{\circ} 6^{\prime} \mathrm{E}, 8$ Aug 1934 , Aleksandrova 134 \& Tiulina (LE); basin of the Kheta river, the Khatanga river, $71^{\circ} 54^{\prime} \mathrm{N}$, $102^{\circ} 6^{\prime}$ E, 16 Aug 1934, Aleksandrova 242 \& Tiulina (LE); Western Taimyr, right side of the Piasina river (middle reaches) the vicinity of the poselok of Tareia, $73^{\circ} 15^{\prime} \mathrm{N}, 90^{\circ} 28^{\prime} \mathrm{E}, 18 \mathrm{Jul} 1965$, Matveeva s. $n$. \& al. (LE); Turukhan Krai, the Piasina river, the mouth of the Pura river (Kuria), 150 verst as away from the mouth of the Piasina, $73^{\circ} 50^{\prime} \mathrm{N}, 87^{\circ} 9^{\prime} \mathrm{E}, 14 \mathrm{Jul}$ 1922, Pushkarev s. n. (LE); Khatanga river, the basin of the Novaya river; moraine ridge on the left side of the Novaya river, $10 \mathrm{~km}$ away from its mouth, $74^{\circ} 17^{\prime} \mathrm{N}, 109^{\circ} 30^{\prime} \mathrm{E}, 19$ Aug 1934, Tiulina 223 (LE); south-eastern Byrranga, $15 \mathrm{~km}$ upstream the mouth of the Malakhaitari, $74^{\circ} 58^{\prime} \mathrm{N}$, 
106³5'E, 28 Jul 1928, Kozhevnikov 154 (LE); Taimyr National Okrug, Khatanga distr, right side of the Kotui river, between the Tompoko and Saamoidin rivers, north-western slope, $71^{\circ} 55^{\prime} \mathrm{N}, 102^{\circ} 5^{\prime} \mathrm{E}, 16$ Aug 1955, Kozlitina s. $n$. (LE); slope of the bank of the Yenisei river near Sarikha sands, 72 $3^{\prime} \mathrm{N}, 82^{\circ} 23^{\prime} \mathrm{E}, 17$ Aug 1914, Kusnezow 2276 \& Reverdatto (LE); Nasonovskiy island at the mouth of the Yenisei river, $70^{\circ} 54^{\prime} \mathrm{N}, 83^{\circ} 11^{\prime} \mathrm{E}$, 24 Aug 1914, Kusnezow 4043 \& Reverdatto (LE); Taimyr, the basin of the Novaya river, Ary-mas natural landmark, $70^{\circ} 50^{\prime} \mathrm{N}, 145^{\circ} 33^{\prime} \mathrm{E}, 15 \mathrm{Jul}$ 1969, Norin s. n. (LE); SE Taimyr, N Anabar plateau, fusion area rivers Fomich and Popigaj, $72^{\circ} 7^{\prime} \mathrm{N}, 110^{\circ} 33^{\prime} \mathrm{E}, 11 \mathrm{Jul} 2008$, Pospelov 08-0466 (MW); central Taimyr, out of the mountains Byrranga p. Fadyukuda, $74^{\circ} 5^{\prime} \mathrm{N}, 96^{\circ} 50^{\prime} \mathrm{E}, 16 \mathrm{Jul}$ 1997, Pospelov 97-376 (MW); Piasina river, the mouth of the Pura river (Kuria), $73^{\circ} 50^{\prime} \mathrm{N}, 87^{\circ} 9^{\prime} \mathrm{E}, 14 \mathrm{Jul} 1922$, Pushkarev s. n. (LE); "Ary-Mas" nature reserve, c. 50-60 km NNW Khatanga, right riverside of Novaya, $72^{\circ} 27^{\prime} \mathrm{N}, 1^{\circ} 1^{\circ} 51^{\prime} \mathrm{E}, 27 \mathrm{Jul} 2004$, Schönswetter s. $n$. \& Tribsch (WU); Minusinsk uyezd, Abakanskaya Inorodnaya Uprava, upper reaches of the Uybata river, 31 Jul 1909, Titov s. n. (LE); Eastern Taimyr, the lower reaches of the Yamu-Nera (the basin of Taimyr Lake), $74^{\circ} 50^{\prime} \mathrm{N}, 106^{\circ} 0^{\prime} \mathrm{E}, 14$ Aug 1928, Tolmachev 770 (C, LE, S); Putorana

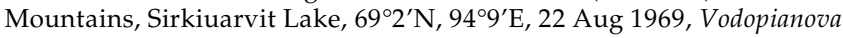
s. n. (LE). Magadan: basin of the Medvezhaia river, downstream of the Chapko river, $61^{\circ} 58^{\prime} \mathrm{N}, 160^{\circ} 56^{\prime} \mathrm{E}, 13$ Aug 1935, Sambuk s. n. \& Zdanevich (LE); Bilibinsky distr., fontes fl. Lelvergyrgyn, $68^{\circ} 3^{\prime} \mathrm{N}, 163^{\circ} 32^{\prime} \mathrm{E}, 21 \mathrm{Jul}$ 1976, Koroleva 5703 \& Petrovsky (C, JE, K, LE, M, MW, NY, US). Nenets Autonomous Okrug: Kanin Peninsula, the Pae-khoi range; source of the Tarcho-Yaga river, 16 Aug 1928, Andreev 1418 (LE); Kolguev Island, valley of the Velikaya river, upstream of the mouth of the Pyrdiu river, $69^{\circ} 20^{\prime} \mathrm{N}, 4^{\circ} 50^{\prime} \mathrm{E}, 31$ Aug 1936, Bogdanovskaya-Gienef s. n. \& Verlichenko (LE); Northern part of Malozemelskaya Tundra, near sopka [Liadak], $67^{\circ} 50^{\prime} \mathrm{N}, 51^{\circ} 0^{\prime} \mathrm{E}, 29$ Jul 1930, Igoshina s. n. (LE); Northern part of Malozemelskaya Tundra, along the bank of the Khorey-Yaga, 67 $50^{\prime} \mathrm{N}$, $51^{\circ} 0^{\prime} \mathrm{E}, 7 \mathrm{Jul} 1930$, Igoshina s. n. (LE); Kanin, northern part, the middle riches of the Peschanka river, $67^{\circ} 19^{\prime} \mathrm{N}, 47^{\circ} 48^{\prime} \mathrm{E}, 12 \mathrm{Jul} 1945$, Matveev s. $n$. (LE); Kolguyev Island, 69 $5^{\prime} \mathrm{N}, 4^{\circ} 15^{\prime} \mathrm{E}, 1902$, Pohle s. $n$. (C, LE); Kanin Peninsula, Krimka, 67 $51^{\prime} \mathrm{N}, 4^{\circ} 49^{\prime} \mathrm{E}, 6$ Aug 1903, Poppius s. n. (FI, P); Kolguev Island, on a slope with meadow on the right side of the Oltsovaya Yakha, left tributary of the Peschanka river, $69^{\circ} 0^{\prime} \mathrm{N}, 49^{\circ} 58^{\prime} \mathrm{E}$, 7 Sep 1930, Smirnova s. n. (LE); Kolguev Island, right side of the Krivaya river, $69^{\circ} 0^{\prime} \mathrm{N}, 48^{\circ} 46^{\prime} \mathrm{E}, 31 \mathrm{Jul} 1930$, Smirnova s. n. (LE). Republic of Karelia: Uusiselkä, Kuivahko niitty, $61^{\circ} 43^{\prime}$ N , 34 ${ }^{\circ} 29^{\prime}$ E, 26 Jul 1942, Kalela s. n. (H). Sakha Republic: lower reaches of the Indigirka, the middle reaches of the Shandrin river, $70^{\circ} 55^{\prime} \mathrm{N}, 151^{\circ} 3^{\prime} \mathrm{E}, 10 \mathrm{Jul} 1974$, Korobkov s. n. \& al. (LE); Tigija, $71^{\circ} 25^{\prime} \mathrm{N}, 8$ Jul 1898, Nilsson s. $n$. (S); Kumach Sor, 713’N, 7 Aug 1898, Nilsson s. n. (S); Balaganach, $71^{\circ} 35^{\prime} \mathrm{N}, 15 \mathrm{Jul} 1898$, Nilsson s. n. (S); lower reaches of the Lena river, Western spurs of the Tuora-Sis range, mouth of the Sietchan river, $71^{\circ} 5^{\prime} \mathrm{N}, 127^{\circ} 30^{\prime} \mathrm{E}, 7 \mathrm{Jul}$ 1956, Norin s. n. \& al. (LE); north end of the Kharaulakhskiy range, right side of the Bykovskaya canal of the Lena river, vicinity of Sokol, $72^{\circ} 20^{\prime} \mathrm{N}, 125^{\circ} 40^{\prime} \mathrm{E}, 15$ Aug 1956, Polozova s. n. \& Yurtsev (LE); Tiksi Bay, NW part of the Buorkhaia Guba (arctic Yakutia), near the airport Tiksi3, flood-plain of the Khorogol river, $71^{\circ} 41^{\prime} \mathrm{N}, 128^{\circ} 54^{\prime} \mathrm{E}, 4$ Aug 2004, Sekretareva s. n. \& Sytin (LE); Lena R. west bank, Chekurovka village, surroundings of settlement, $71^{\circ} 3^{\prime} \mathrm{N}, 127^{\circ} 31^{\prime} \mathrm{E}, 12 \mathrm{Jul} 2004$, Solstad $04 / 0266$ A \& Elven (O); lower reaches of the Lena river, Tit-Ary Island, $71^{\circ} 58^{\prime} \mathrm{N}, 127^{\circ} 0^{\prime} \mathrm{E}, 4$ Aug 1956, Tolmachev s. n. E al. (LE); lower reaches of the Lena river between Tit-Ary Island and Stolb Island, right side of the Lena, $10 \mathrm{~km}$ downstream of Tit-Ary, $71^{\circ} 50^{\prime} \mathrm{N}, 1^{\circ} 7^{\circ} 20^{\prime} \mathrm{E}, 31 \mathrm{Jul} 1955$, Vasilkova s. n. \& al. (LE); lower reaches of the Lena river, Polar station Sokol, Bykovskaya Canal, $72^{\circ} 24^{\prime} \mathrm{N}, 126^{\circ} 48^{\prime} \mathrm{E}$, 28 Jul 1955, Vasilkova s. n. \& al. (LE). Sakhalin: Kuril Islands, 30 Aug 1929, Bergman 237 (GH); Kuril Archipelago, Simushir Island, inland coastal margin of Malaya Bay, $47^{\circ} 5^{\prime} \mathrm{N}, 1^{\circ} 2^{\prime} \mathrm{E}$, 18 Aug 1995, Gage SG1427 (NY); Kuril Archipelago, Onekotan Island, Kol'tsyevoi Spine, rim of crater, Lake Kol'tsyevoi, 49²2'N, 15444'E, 7 Aug 1996, Gage SG1976 (NY); Kuril Archipelago, Kharimkotan, Severgina Bay, ca. $1 \mathrm{~km}$ inland from shoreline, $49^{\circ} 9^{\prime} \mathrm{N}$, 1542을, 8 Aug 1996, Gage SG2021 (NY); Kuril Archipelago, Onekotan Island, southern end of island, near Trudny River, $49^{\circ} 16^{\prime} \mathrm{N}, 154^{\circ} 44^{\prime} \mathrm{E}, 9$ Aug 1996, Gage SG2117 (NY); Kuril Archipelago, Paramushir, NE corner of the island, environs of lake fed by Savushkina River, 5044'N, 156 ${ }^{\circ}$ 'E, 4 Aug 1997, Gage SG4139 (NY); Kuril Archipelago, Shumshu, inland from Babushkina Bay, environs of Luzhanka river, 50 $38^{\prime} \mathrm{N}, 156^{\circ} 24^{\prime} \mathrm{E}, 10$ Aug 1997, Gage SG4274 (NY); Kuril Archipelago, Paramushir, inland from eastern Tukharka, Cape Baklanyi, $50^{\circ} 10^{\prime} \mathrm{N}, 155^{\circ} 38^{\prime} \mathrm{E}, 17$ Aug 1997, Gage SG4492 (NY); Kuril Archipelago, Kharimkotan, Severgina Bay, 49 $9^{\prime} \mathrm{N}, 1^{\circ} 4^{\circ} 28^{\prime} \mathrm{E}, 8$ Aug 1996, Semsrott BS0173 (NY); Kuril Archipelago, Makanrushi island, inland from Zakat Bay, environs of Cape Poludennyi, 49 $44^{\prime} \mathrm{N}, 1^{\circ} 4^{\circ} 24^{\prime} \mathrm{E}, 18$ Aug 1997, Semsrott BS1487 (NY). Yamalo-Nenets Autonomous Okrug: Yamal Peninsula, the shore of Obskaya
Guba, $30 \mathrm{~km}$ to the N of Cape Kamennyi, $68^{\circ} 30^{\prime} \mathrm{N}, 73^{\circ} 34^{\prime} \mathrm{E}$, Aug 1971, Gaev s. $n$. \& Fediakov (LE); Gydansk Peninsula, the lower reaches of the Khalmeriakha river, $71^{\circ} 30^{\prime} \mathrm{N}, 78^{\circ} 5^{\prime} \mathrm{E}, 18$ Jul 1988, Khitun 8120 (LE); 23 Jul 1988, Khitun 8151 (LE); Gydansk Peninsula, the lower reaches of the Khalmeriakha river,valley wall of Lang-to lake, $71^{\circ} 30^{\prime} \mathrm{N}, 78^{\circ} 5^{\prime} \mathrm{E}, 30 \mathrm{Jul}$ 1988, Khitun 8234 (LE).

U. S. A. Alaska: White Mountain between the headwaters of Sheep and Mascot Creeks, 654ㄴ ${ }^{\prime} \mathrm{N}, 147^{\circ} 6^{\prime} \mathrm{W}, 1$ Jul 1953, Gjaerevoll 294 (S); Norton Sound, behind Pastolik, $63^{\circ} 8^{\prime} \mathrm{N}, 163^{\circ} 18^{\prime} \mathrm{W}, 21$ Jul 1926, Porsild 893 \& Porsild (H); Seward Peninsula, Port Clarence, Teller, $65^{\circ} 16^{\prime} \mathrm{N}, 166^{\circ} 20^{\prime} \mathrm{W}, 24$ Aug 1926 , Porsild 1414 \& Porsild (S); Bering Strait, Port Clarence, Teller, $65^{\circ} 15^{\prime} \mathrm{N}$, 166²1'W, Aug 1949, Scamman 5410 (S).

Notes-Trisetum sibiricum is the most polymorphic and widespread taxon of the section. This species presents a great morphological variability throughout its distribution area. The panicle shape, as well as the size of the individuals, is a quite variable character.

Although T. sibiricum is a distinct species, it has been constantly confused with $T$. flavescens. Trisetum flavescens is another species of the genus with a wide distribution, covering the whole of Europe and the Maghrebian mountains of North Africa, being introduced in many other temperate zones. Trisetum flavescens belongs to $T$. sect. Trisetum, and it is characterized by having green (rarely purple shiny) panicles, twisted and geniculate awns, and leaves narrower and with a more compact venation that T. sibiricum. However, T. sibiricum belongs to T. sect. Sibirica, and it is differentiated from $T$. flavescens, apart from the characters mentioned before, by its golden brownish or yellowish panicles and twisted but not geniculate awns (Tzvelev 1976; Frey 1992).

The westernmost specimens of $T$. sibiricum are those from Poland and Serbia, the ones from Serbia named Avena rufescens by Pančić (1884). Some authors (i.e. Roshevitz 1922; Tzvelev 1976; Jonsell 1980) also placed this species in Romania. In the Flora of Romania by Sãvulescu (1972), this species is not cited. Moreover, we have not identified any of the herbarium material from this country as T. sibiricum.

Owing to the polymorphism of T. sibiricum, some subspecies or varieties have been described by different authors. Kitagawa (1940) described a new variety, T. sibiricum var. umbratile from Manchuria, characterized by its smaller 2-flowered spikelets (5 mm long) and shorter awns (6.5 mm long). Later, according to these characters, Kitagawa (1956) and Wu and Phillips (2006) recognized it as a species, whereas Tzvelev (1976) identified it as a subspecies, separating it from T. sibiricum subsp. sibiricum by its wider leaves, and also leaves and basal leaf-sheaths indumentum. Keng described a new species from central China, T. pauciflorum, indicating that it was close to T. henryi (Anonymous 1976). This species has small spikelets, as T. umbratile, but with recurved awns and a different distribution. $\mathrm{Wu}$ and Phillips (2006) indicated that it was not a well-known species, close to T. umbratile. This range of variation in spikelet size and awn length falls well within the variation range of $T$. sibiricum subsp. sibiricum; along its distribution, specimens with smaller and 2-flowered spikelets have been studied, mainly in material from Mongolia. For this reason, and also following Stuessy et al. (2014), who recognized geography as a fundamental component for recognition of infraspecific taxa, $T$. umbratile and T. pauciflorum have been considered as synonyms of $T$. sibiricum subsp. sibiricum.

However, another recognized subspecies, T. sibiricum subsp. litorale, is a smaller plant with shorter and denser panicles, usually ovate in outline, distributed along northern Russia, extending to Alaska. In the studied material, specimens from Altai show more contracted panicles, but not as much as those of the coastal regions, and also without shorter culms. This 
subspecies has a constant morpho-geographic pattern, which overlaps with the morphology of T. sibiricum subsp. sibiricum in Kuril Archipelago and Kamchatka Peninsula (where both subspecies overlap). As did Roshevitz (1922) and Tzvelev (1976), we have recognized it at subspecific rank.

Hooker (1897) described the species Avena sikkimensis from the Himalayan Mountains of Sikkim state in India, synonymized into Trisetum flavescens by Bor (1956). Chrtek (1968) indicated that $A$. sikkimensis is different from $T$. flavescens, mainly by its not geniculate awns, combining it as Trisetum sikkimensis, and including it in serie Sibirica. As indicated before, $T$. flavescens and $T$. sibiricum have been frequently confused, and the specimens from Sikkim correspond to $T$. sibiricum. Apart from its slightly smaller stamens, which have also been observed in a specimen from Mongolia [Komarov s. $n$. (LE)], we did not find more differences between T. sikkimensis and T. sibiricum subsp. sibiricum. For this reason, T. sikkimensis has been considered a synonym of $T$. sibiricum.

2. Trisetum turcicum Chrtek, Bot. Not.119: 487. 1966. Trisetaria turcica (Chrtek) Banfi \& Soldano, Atti Soc. Ital. Sci. Nat. Mus. Civico Storia Nat. Milano 135: 386. 1996._TYPE: TURKEY. Lazistan, Djimil valley, $40^{\circ} 40^{\prime} \mathrm{N} 39^{\circ} 40^{\prime} \mathrm{E}$, Aug 1866, B. Balansa 1551 (holotype: PRC-454965 (image!); isotypes: G-176299!, G-176300!, GH!, GOET-6970!, JE-18873!, K-808699!, LE-9412!, LE-9413!, P-3644578!, P-3645245!, P-3351081!, P-3351082!, P-3351137!, P-2248780!, P-2248781!, RO (image!), US-1064357!, W-1889-0033667!).

Trisetum thospiticum Chrtek, Bot. Not.119: 489. 1966. Trisetaria thospitica (Chrtek) Banfi \& Soldano, Atti Soc. Ital. Sci. Nat. Mus. Civico Storia Nat. Milano 135: 386. 1996.-TYPE: TURKEY. Bitlis province, Suphan Dag, 38 $57^{\prime} \mathrm{N} 42^{\circ} 50^{\prime} \mathrm{E}$, 28 Sep 1954, P. H. Davis 24763 a E O. Polunin (holotype: E-196474 (image!); isotypes: BM-959386!, K-808700!).

Herb (13.4-)43.7-87.7(-102) cm high, laxly caespitose, shortly rhizomatous, culm $0.7-1.3 \mathrm{~mm}$ diam, glabrous, rarely pubescent on the upper part; nodes $4-5$, separated along the culm, enclosed or not by the sheaths, puberulous. Basal leafsheaths glabrous to slightly pubescent, with hairs up to $0.2 \mathrm{~mm}$ long, decaying in fibers, yellowish to greenish or brownish; basal leaf-blades (6-)7.5-10 × 2.6-3.3 mm, flat, usually rolled when dried, from glabrous to pubescent abaxially and adaxially, margins with hairs $0.1-0.8(-1.2) \mathrm{mm}$ long, greenish to brownish; top culm leaf-sheaths (1-)1.4-1.8(-2) cm long, shorter than the internodes, rarely the same length, glabrous to pubescent, with or without cilia on the margins; top culm leafblades (6.3-)7-12(-14) cm long $\times 3-4.2(-5.5) \mathrm{mm}$ wide, flat, pubescent abaxially and adaxially, ciliolate, with hairs $0.1-$ 0.7(-0.9) mm long, greenish to greyish, sometimes brownish; inner collar region hairy, with scattered hairs $0.05-0.3 \mathrm{~mm}$ long, also on the margins, rarely glabrous; ligules $1.5-2.8(-3.4) \mathrm{mm}$ long, slightly laciniate to laciniate, glabrous, with scattered hairs $0.1-0.3 \mathrm{~mm}$ long on the upper part. Basal node of the panicle glabrous. Panicles (7.2-)8.2-13.4(-15) cm long $\times 2$ $3.6(-4) \mathrm{cm}$ wide, elliptic to oblong, sometimes narrowly to broadly elliptic, dense, sometimes more or less lax; rachis glabrous to slightly pubescent, with hairs up to $0.08 \mathrm{~mm}$ long; branches in whorls of 3 or 4 , longest basal branches 13.2(-3.5) cm long. Spikelets 7.4-9.7(-10.5) $\mathrm{mm}$ long $\times(2-) 2.7-$ 4.4(-4.6) $\mathrm{mm}$ wide, 2-3-flowered; pedicels $2.7-5.7 \mathrm{~mm}$ long, slightly pubescent to pubescent, with hairs up to $0.1 \mathrm{~mm}$ long. Glumes unequal [ratio of lower glume length/upper glume length $=(0.63-) 0.7-0.82(-0.9)]$; lower glume $(4-) 5-7.2 \mathrm{~mm}$ long $\times 0.9-1.2(-1.5) \mathrm{mm}$ wide, narrowly lanceolate to lanceolate [ratio of lower glume width/lower glume length = 0.16-0.22(-0.24)], acuminate to long acuminate, 1(-2)-nerved, glabrous, usually with hairs up to $0.08 \mathrm{~mm}$ long on the upper part of the margins and central nerve, greenish on the central part surrounded by yellowish; upper glume (6.5-)7.2-8 (-8.7) $\mathrm{mm}$ long $\times(1.6-) 1.8-2.2 \mathrm{~mm}$ wide, elliptic to oblanceolate, sometimes lanceolate [ratio of upper glume width/ upper glume length $=(0.23-) 0.25-0.28(-0.3)]$, acuminate to long acuminate, 3-nerved, glabrous, with hairs up to $0.08 \mathrm{~mm}$ long from the middle to the upper part of the midrib and margins, greenish with yellowish; rachilla segments between first and second floret 1.7-2.2 mm long, with hairs 1-2 mm long; rachilla segments to sterile floret 1.5-2 mm long, with hairs $0.3-1.2 \mathrm{~mm}$ long. Lemmas $6.5-8.7 \mathrm{~mm}$ long $\times 1.6-2 \mathrm{~mm}$ wide, narrowly elliptic to lanceolate, sometimes slightly oblanceolate (ratio of lemma width/lemma length $=0.19-0.28$ ), scabridulous, with hairs up to $0.1 \mathrm{~mm}$ long, brownish to greenish, sometimes yellowish; callus $0.2-0.3 \mathrm{~mm}$ long, orbicular, rarely elliptic, glabrous or with scattered hairs $0.1-0.2 \mathrm{~mm}$ long; apical teeth $0.2-0.9 \mathrm{~mm}$ long, with aristules 0.8-1.5(-2) mm long; awn (8.8-)9.5-12.5(-13.4) mm long, inserted (3.8-)4-4.8 $\mathrm{mm}$ from the base (ratio of awn insertion from the base length/lemma length $=(0.57-) 0.6-0.68)$, geniculate below the middle, slightly twisted to twisted near the base, with adpressed hairs $0.05 \mathrm{~mm}$ long. Paleas (5-)5.7$7(-7.2) \mathrm{mm}$ long $\times 1.5-1.8 \mathrm{~mm}$ wide [ratio of palea length/ lemma length $=0.74-0.84(-0.93)]$, elliptic, sometimes oblanceolate, margins with short hairs from the middle till the upper part; teeth $0.3-0.4(-0.7) \mathrm{mm}$ long, with short antrorse hairs. Lodicules $0.5-1 \mathrm{~mm}$ long, with two bifid lobules, glabrous. Anthers (3-)3.3-4 mm long. Ovary 0.4-1(-1.4) mm long. Caryopsis $3.3-4 \mathrm{~mm}$ long $\times 0.6 \mathrm{~mm}$ wide, narrowly elliptic to oblong. Figure 4.

Chromosome Number-2n $=28$ (Tzvelev 1976).

Phenology-Flowering and fruiting from July to September.

Distribution and Habitat-It is distributed from the mountains of south and eastern Turkey to the Caucasus and northwest Iran; 2,000-2,800 m of elevation; in forest or among shrubs in subalpine meadows. Figure 5.

Specimens Examined-ARMENIA. Gegharkunik: Nor-Bajazet distr., prope pag. Alekssandrovka, $40^{\circ} 30^{\prime} \mathrm{N}, 44^{\circ} 58^{\prime} \mathrm{E}, 8$ Jul 1929, Zedelmejer $\mathcal{E}$ Hejdemann s. n. (LE). Kotayq: vicinity of Gokcha Lake, Darachichag, $40^{\circ} 23^{\prime} \mathrm{N}, 45^{\circ} 19^{\prime} \mathrm{E}, 1 \mathrm{Jul}$ 1923, Smirnov 119 (MW). Shirak: Alagez, Karnalykh gorge, south-east of Ziarat mount, $40^{\circ} 32^{\prime} \mathrm{N}, 44^{\circ} 4^{\prime} \mathrm{E}, 9$ Aug 1932, Busch s. $n$. (LE). Syunik: on a slope to the valley of the Vargavar-chay River, $38^{\circ} 53^{\prime} \mathrm{N}$, $46^{\circ} 15^{\prime} \mathrm{E}, 1 \mathrm{Jul} 1956$, Egorova 1685 \& al. (LE); along a slope to the bed of a right tributary of the Megriget River upwards of Vardanadzor, $38^{\circ} 53^{\prime} \mathrm{N}, 46^{\circ} 15^{\prime} \mathrm{E}$, 4 Jul 1956, Egorova 1844 \& al. (LE). Yerevan: Ketan-Dag, $40^{\circ} 21^{\prime} \mathrm{N}, 44^{\circ} 41^{\prime} \mathrm{E}$, 18 Aug 1930, Acheev s. n. (MW).

AZERBAIJAN. Nakhichevan: monte Arazhon, $39^{\circ} 17^{\prime} \mathrm{N}, 45^{\circ} 47^{\prime} \mathrm{E}, 3$ Aug 1933 , Gadzhiev s. n. et al. (LE); inter p. Aravsa et monte Arazhon, $39^{\circ} 17^{\prime} \mathrm{N}$, $45^{\circ} 47^{\prime} \mathrm{E}, 29 \mathrm{Jul}$ 1933, Gadzhiev s. n. et al. (LE); 5-7 km ad NO Urmis, $39^{\circ} 4^{\prime} \mathrm{N}$, $45^{\circ} 58^{\prime}$ E, 4 Aug 1933, Karjagin s. n. \& Isaev (F).

GEORGIA. Akhmetis Raioni, m. Diklo, $42^{\circ} 23^{\prime} \mathrm{N}, 45^{\circ} 41^{\prime} \mathrm{E}, 29$ Jul 1861, Ruprecht s. n. (LE).

IRAN. Āz̄ārbāyjān-e Gharbī: Zanjan distr., Tachte Balküns, $36^{\circ} 36^{\prime} \mathrm{N}$ $47^{\circ} 16^{\prime} \mathrm{E}$, 18 Aug 1884, Knapp s. n. (WU).

RUSSIA. Dagestan: Maara mountain, ad pagum Akusha, $42^{\circ} 16^{\prime} \mathrm{N}$, $47^{\circ} 20^{\prime} \mathrm{E}, 16 \mathrm{Jul} 1898$, Alexeenko s. n. (LE). Kabardino-Balkar Republic: BashilAuz, the upper reaches of the Chegem, $43^{\circ} 17^{\prime} \mathrm{N}, 43^{\circ} 8^{\prime} \mathrm{E}, 20 \mathrm{Jul} 1913$, Bush s. n. \& Bush (LE); Karasu, Kara-Chiran glacier, $43^{\circ} 17^{\prime} \mathrm{N}, 43^{\circ} 24^{\prime} \mathrm{E}, 29 \mathrm{Jul} 1925$, Bush 34 \& Bush (LE). North Ossetia-Alania: on Tsadanzerta by the lower edge of Chefanzara, $43^{\circ} 11^{\prime} \mathrm{N}, 44^{\circ} 14^{\prime} \mathrm{E}, 14$ Aug 1925, Bush 42 \& Bush (LE); Terek oblast, Aday-khokh, $42^{\circ} 56^{\prime} \mathrm{N}, 4^{\circ} 39^{\prime} \mathrm{E}, 28 \mathrm{Jul}$ 1900, Markovich s. n.; Terek oblast, Daj-chveh, $42^{\circ} 56^{\prime} \mathrm{N}, 44^{\circ} 39^{\prime} \mathrm{E}, 29$ Jul 1900, Markovich s. $n$. (JE). 

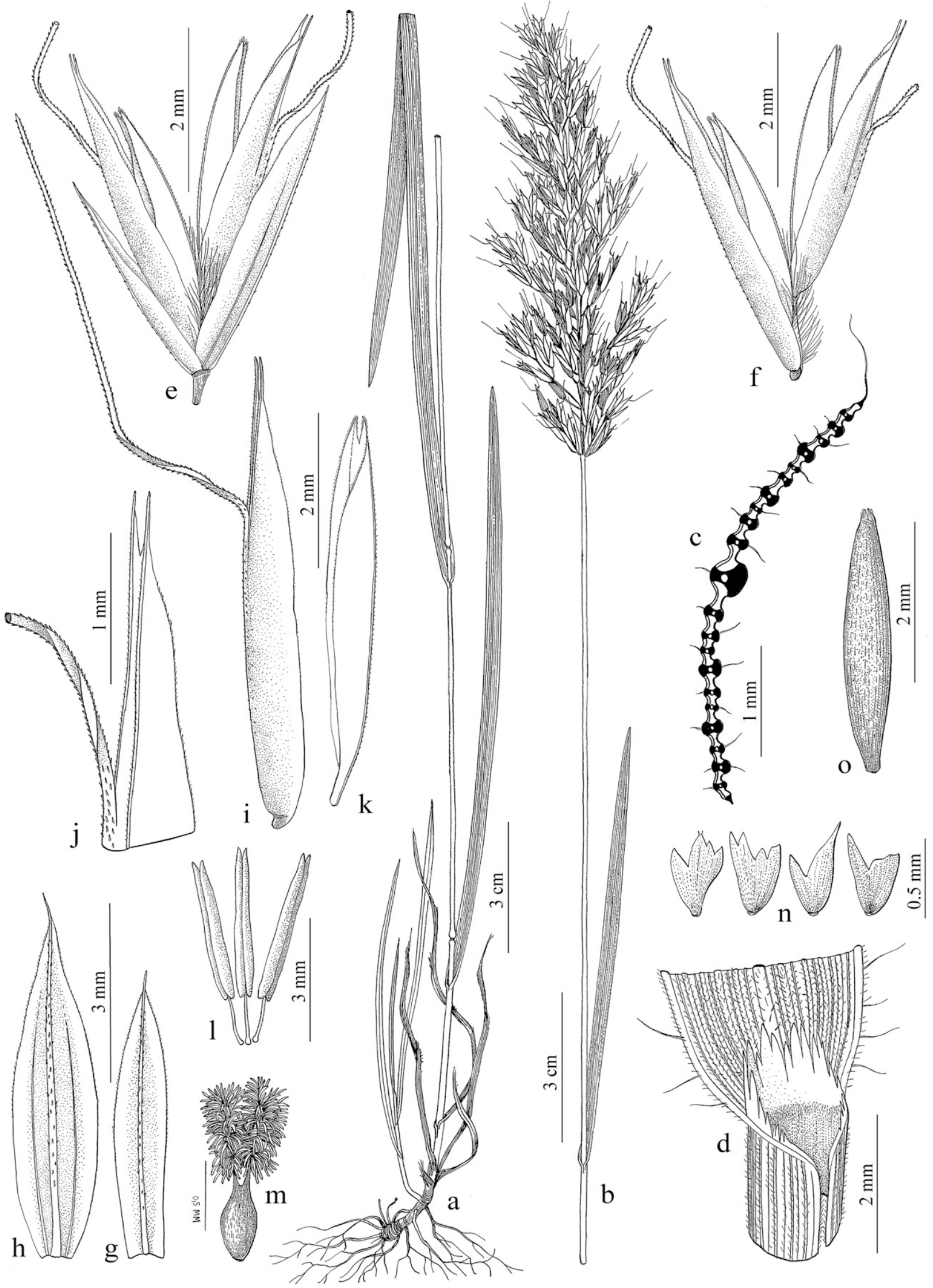

FIg. 4. Trisetum turcicum. a. Basal habit. b. Inflorescence. c. Transverse section of leaf-blade. d. Sheath, ligule, and portion of the blade. e. Spikelet. f. Florets. g. Lower glume, dorsal view. h. Upper glume, dorsal view. i. Floret. j. Lemma, upper part, lateral view. k. Palea, lateral view. 1. Stamens. m. Pistil. n. Lodicules. o. Caryopsis. (Balansa 1551, G-00176299, a-b; Davis 46563, K, c; Balansa 848, P-02243990, d-f, i-m; Sintenis 1142, JE, g-h, o). 


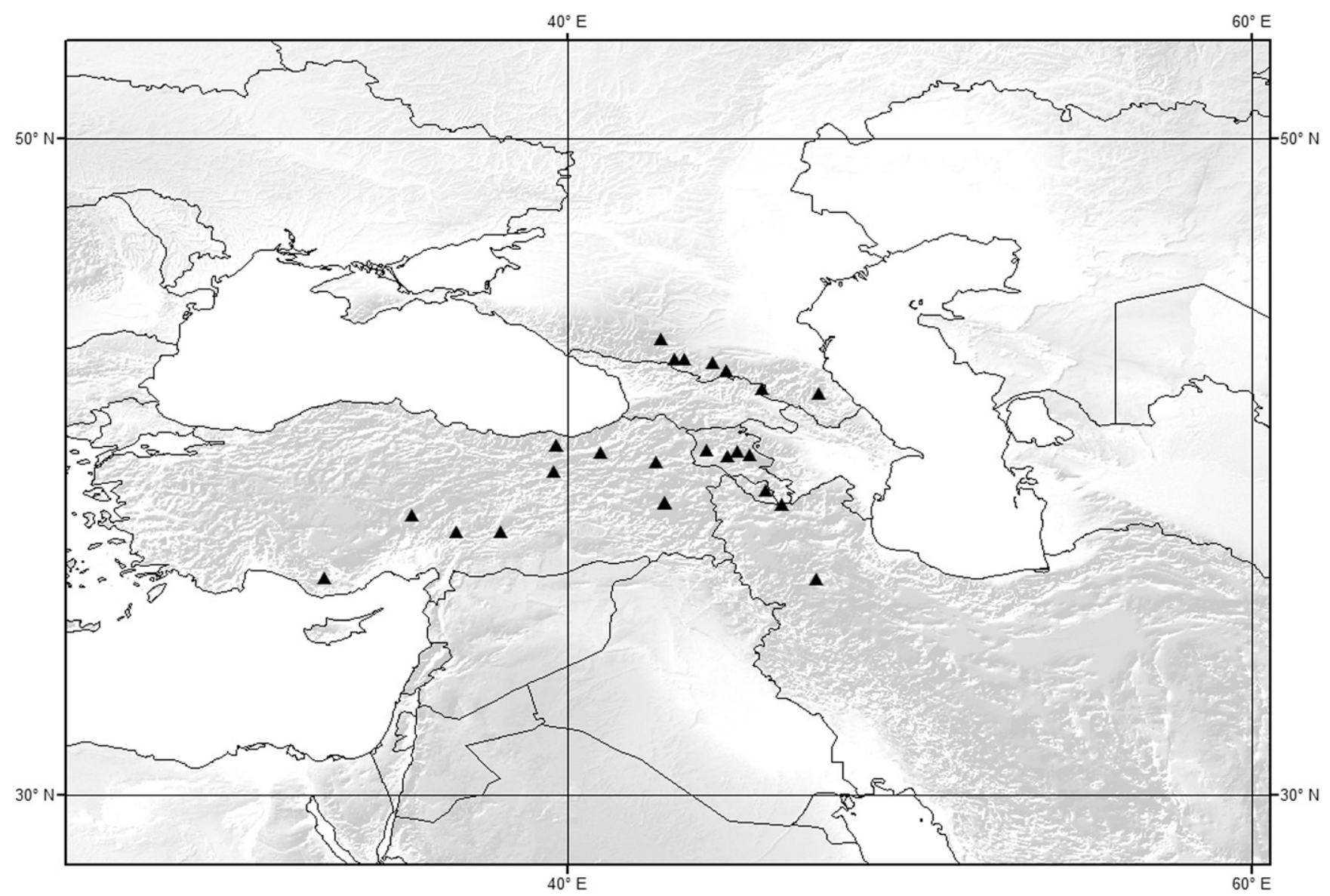

Fig. 5. Distribution of Trisetum turcicum.

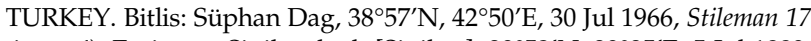
(E, image!). Erzincan: Sipikordagh [Sipikor], 39² $52^{\prime} \mathrm{N}, 39^{\circ} 35^{\prime} \mathrm{E}, 5 \mathrm{Jul} 1889$, Sintenis 1142 (JE). Erzurum: between Erzurum and Ispir, East of Rirekent village, Halbogari valley, $40^{\circ} 28^{\prime} \mathrm{N}, 40^{\circ} 59^{\prime} \mathrm{E}, 14$ Aug 1976, Tatli 5331 (GOET). Kahramanmaraş: Beryt Dagh, $38^{\circ} 3^{\prime} \mathrm{N}, 36^{\circ} 46^{\prime} \mathrm{E}, 9$ Aug 1865, Haussknecht s. n. (JE). Karaman: près Ermenek, 36 $38^{\prime} \mathrm{N}$, 32 $53^{\prime} \mathrm{E}$, Jul 1872, Péronin 214 (MPU, P, S). Kars: $6 \mathrm{~km}$ from Sarikamis to Karakurt, $40^{\circ} 9^{\prime} \mathrm{N}, 42^{\circ} 36^{\prime} \mathrm{E}, 15 \mathrm{Jul}$ 1966, Davis 46563 (K, US). Kayseri: Mount Argée [Erciyes], 38 32’ N, $35^{\circ} 27^{\prime} \mathrm{E}, 9$ Jul 1856, Balansa 848 (G, GOET, L, P). Malatya: inter urbem Malatja et vicum Kjachta, in declivibus opimis montis Gök Tepe versus Kumik, 38³’N, 38²'E, 16 Jul 1910, Handel-Mazzetti 2279 (W). Rize: Djimil valley, $40^{\circ} 40^{\prime} \mathrm{N}, 39^{\circ} 40^{\prime} \mathrm{E}, 21$ Aug 1866, Balansa s. n. (L).

Notes-The Caucasian specimens of T. turcicum have been sometimes confused with $T$. sibiricum. Trisetum turcicum is mainly differentiated from $T$. sibiricum, and also from the rest of species of $T$. sect. Sibirica, by its longer anthers and its awns geniculate below the middle.

Apart from T. turcicum, Chrtek (1966) described another species of Trisetum from Eastern Turkey, T. thospiticum. This species was separated from T. turcicum by its habit, smaller spikelets, and shorter anthers. The habit of the type specimen studied [Davis 24763 \& Polunin (K-808700)] has been also observed in specimens of T. turcicum from Azerbaijan [Karjagin s. n. $\mathcal{E}$ Isaev (F)], which are slightly densely caespitose and with more remains of dried basal leaves. The length of spikelets and anthers of the type specimen of T. thospiticum $(9.7 \mathrm{~mm}$ and $3.5 \mathrm{~mm}$ long, respectively) fall into the variability of $T$. turcicum [7.4-9.7(-10.5) mm and (3-)3.3-4 mm long, respectively)]. For those reasons, $T$. thospiticum has been considered as synonyms of T. turcicum.
As indicated before, Chrtek (1968) separated the Asiatic species of Trisetum section Trisetum in two series (ser. Trisetum and ser. Sibirica) according to the awn shape. He included $T$. turcicum and T. thospiticum in ser. Trisetum, because of its geniculate awn. In this work, T. turcicum has been included in $T$. sect. Sibirica because it shows the rest of the characters of the section, being very close to $T$. sibiricum.

3. Trisetum Bifidum (Thunb.) Ohwi, Bot. Mag. (Tokyo) 45: 191. 1931. Bromus bifidus Thunb., Syst. Veg. ed. 14: 119. 1784. Avena bifida (Thunb.) P. Beauv., Ess. Agrostogr.: 89, 155. 1812. Trisetum flavescens var. bifidum (Thunb.) Makino, Bot. Mag. (Tokyo) 26: 215. 1912 ["bifidus"].-TYPE: JAPAN. probably near Nagasaki, C. P. Thunberg s. n. (lectotype, designated by Veldkamp \& van der Have (1983: 129): UPS-THUNB-002576 (image!); isolectotypes: L-50296!, P-2255904!, S-13-31156!, S-13-31784!).

Bromus avenaeformis Steud., Syn. Pl. Glumac. 1: 326. 1854.TYPE: JAPAN. [without locality], H. Bürger s. n. (lectotype, designated by Veldkamp \& van der Have (1983: 129): L-908.98-239 (image!); isolectotype: P-740331!).

Trisetum flavescens var. macranthum Hack., Bull. Herb. Boissier 7: 703. 1899. Trisetum macranthum (Hack.) Keng, Sinensia 11: 411. 1940. Trisetum bifidum var. macranthum (Hack.) Ohwi, J. Jap. Bot. 17: 445. 1941.—TYPE: JAPAN. Hokkaido Island, Ishikari Prov., Sapporo, $43^{\circ} 03^{\prime} \mathrm{N} 141^{\circ} 20^{\prime} \mathrm{E}$, U. J. Faurie 7191 (lectotype, here designated: P-2255899!; isolectotypes: FI!, W-1916-0031816!). 
Trisetum flavescens var. papillosum Hack., Bull. Herb. Boissier 7: 702. 1899. Trisetum sibiricum subsp. papillosum (Hack.) Roshev., Izv. Glavn. Bot. Sada R. S. F. S. R. 21: 2. 1922. Trisetum bifidum var. papillosum (Hack.) Ohwi, J. Jap. Bot. 17: 445. 1941.-TYPE: JAPAN. Tokyo, 35²41’N $139^{\circ} 41^{\prime} \mathrm{E}, 6$ Jun 1888, U. J. Faurie 2359 (lectotype, designated by van Royen (1980: 1158): P-2255912!; isolectotypes: P-3351083!, US-101513!).

Trisetum taquetii Hack., Repert. Spec. Nov. Regni Veg. 12: 386. 1913.-TYPE: SOUTH KOREA. Jeju region, Quelpaert Island, forest of Hallasan, $33^{\circ} 24^{\prime} \mathrm{N} 126^{\circ} 32^{\prime} \mathrm{E}$, Jul 1909, T. Taquet 3403 (holotype: W-1916-0042226!; isotypes: AAH!, C!, LE-1001930!, NY!, TI-21900 (image!), TI-21901 (image!), US-1128818!, US-3619058!).

Trisetum biaristatum Nakai, Bot. Mag. (Tokyo) 35: 150. 1921. Trisetum bifidum var. biaristatum (Nakai) Ohwi, J. Jap. Bot. 17: 445. 1941.-TYPE: JAPAN. Izu province, Aogashima Island, $32^{\circ} 28^{\prime} \mathrm{N} 139^{\circ} 46^{\prime} \mathrm{E}, 16$ Jun $1920, N$. Matsuzaki s. $n$. (holotype: TI-21892 (image!)).

Trisetum bifidum f. contracta Ohwi, Bot. Mag. (Tokyo) 45: 192. 1931.-TYPE: NORTH KOREA. P'yŏngan province, Nampo, $38^{\circ} 44^{\prime} \mathrm{N} 125^{\circ} 24^{\prime} \mathrm{E}$, Jul 1901, U. J. Faurie 883 (lectotype, here designated: P-2255887!; isolectotypes: G-443048!, P-2255946!, W-1916-0031813!).

Trisetum bifidum var. oshimense Honda, Bot. Mag. (Tokyo) 49: 697. 1935.-TYPE: JAPAN. Honshû Island, Izu province, Ôshima, Sashikiji, 344 $41^{\prime} \mathrm{N} 139^{\circ} 25^{\prime} \mathrm{E}, 22$ May 1933, Y. Jôtani s. n. (holotype: TI-21893 (image!)).

Trisetum bifidum var. viride Honda, Bot. Mag. (Tokyo) 49: 697. 1935.-TYPE: JAPAN. Honshû Island, Izu province, Ôshima, Motomura, $34^{\circ} 45^{\prime} \mathrm{N}$ 139 $21^{\prime} \mathrm{E}, 12$ Jun 1932, Y. Jôtani s. n. (holotype: TI-21894 (image!)).

Herb (32.8-)44-64.5(-114) cm high, densely tufted, shortly rhizomatous, culm (0.4-)0.7-1.2(-1.5) $\mathrm{mm}$ diam, glabrous; nodes (2-)3-5, separated along the culm, not enclosed by the sheaths, glabrous. Basal leaf-sheaths glabrous to pubescent, with hairs (0.05-)0.5-1(-1.5) $\mathrm{mm}$ long, decaying into fibers, yellowish to brownish; basal leaf-blades (0.38-)0.53$1.4(-1.85) \times(0.21-) 0.28-0.41(-1.15) \mathrm{mm}$, flat, sometimes slightly rolled when dried, glabrous adaxially, glabrous to puberulous mainly on the nerves abaxially, margins with hairs $0.05-1 \mathrm{~mm}$ long, greenish and brownish, sometimes yellowish; top culm leaf-sheaths (11.2-)12-20(-27) cm long, shorter than the internodes, rarely longer, glabrous, ciliolated, with hairs up to $1 \mathrm{~mm}$ long; top culm leaf-blades (2.6-)4-10(-13) $\mathrm{cm}$ long $\times$ $(0.2-) 0.27-0.53(-0.78) \mathrm{cm}$ wide, flat, sometimes parallel to the culm, rarely extending to the panicle, with the central nerve similar to the lateral ones, glabrous to pubescent adaxially and abaxially, with short, rarely long hairs $0.05-0.1(-1.1) \mathrm{mm}$ long, greenish to greyish; inner collar region glabrous to hairy, with scattered hairs (0.1-)0.25-0.5(-0.8) mm long; ligules 0.41.1(-1.6) $\mathrm{mm}$ long, apex denticulate, rarely slightly lobulate, glabrous, sometimes slightly pubescent or with scattered hairs abaxially, with hairs 0.05-0.1(-0.2) mm long. Basal node of the panicle glabrous. Panicles (8.5-)11-19(-27) cm long $\times(2.3-) 2.8-$ $3.5(-5) \mathrm{cm}$ wide, oblong to oblong-lanceolate in outline, sometimes elliptic-oblong; rachis glabrous to slightly pubescent on the upper part, with patent and antrorse hairs up to $0.1 \mathrm{~mm}$ long; branches in whorls of 3 or 4 , longest basal branches
(0.9-)1.6-4.7(-7) mm long. Spikelets (6.5-)7.5-9.2(-11.1) $\mathrm{mm}$ long $\times(1.8-) 3-4.3(-5) \mathrm{mm}$ wide, 2-3(-4)-flowered; pedicels (2.3-)3.2-5.4(-7.5) $\mathrm{mm}$ long, with scattered hairs or pubescent, with hairs up to $0.1 \mathrm{~mm}$ long, rarely glabrous. Glumes unequal [ratio of lower glume length/upper glume length = (0.11-)0.48-0.59(-0.67)]; lower glume (0.7-)2.8-3.6(-4.3) $\mathrm{mm}$ long $\times(0.2-) 0.6-0.9 \mathrm{~mm}$ wide, narrowly lanceolate, rarely broadly lanceolate [ratio of lower glume width/lower glume length $=(0.16-) 0.19-0.25(-0.29)]$, acute to acuminate, rarely long acuminate, 1-nerved, glabrous, with hairs $0.05-0.1(-0.2) \mathrm{mm}$ long from the center to the upper part of the nerve, greenish on the center, rarely with some purplish on the upper part; upper glume (5.3-)5.5$6.5(-7.7) \mathrm{mm}$ long $\times(1.6-) 1.8-2(-2.2) \mathrm{mm}$ wide, lanceolate to elliptic, sometimes oblong [ratio of upper glume width/ upper glume length $=(0.25-) 0.29-0.33(-0.37)]$, acute to acuminate, 3-nerved, glabrous, with hairs $0.1-0.2 \mathrm{~mm}$ long from the middle to the upper part of the central nerve, sometimes also on the upper part of the margins, greenish with a yellowish border, sometimes purplish on the margins to the top; rachilla segments between first and second floret 1.1-1.5(-1.8) $\mathrm{mm}$ long, with hairs (0.2-)1.2-1.4(-1.8) mm long; rachilla segments to sterile floret (1-)1.6-1.8(-2.1) mm long, with hairs $(0.1-) 0.35-0.7(-1) \mathrm{mm}$ long. Lemma (6-)6.5-7.5(-8.2) mm long $\times(0.2-) 0.6-0.9 \mathrm{~mm}$ wide, narrowly lanceolate to narrowly oblong [ratio of lemma width/lemma length $=(0.18-) 0.2-0.24(-0.27)]$, punctate-scabrid, with longer hairs up to $0.2 \mathrm{~mm}$ long on the central and upper part, brownish to yellowish, sometimes greenish; callus $0.2-0.3 \mathrm{~mm}$ long, orbicular to elliptic, with hairs $0.3-0.7 \mathrm{~mm}$ long; apical teeth $0.1-0.4(-0.8) \mathrm{mm}$ long, with aristules (0.1-)0.6-1(-1.3) mm long; awn (7-)8.2-10.6(-12) mm long, inserted (3.5-)4.1-5.3(-6) $\mathrm{mm}$ from the base [ratio of awn insertion from the base length/lemma length $=(0.56-) 0.62-$ $0.74(-0.83)]$, strongly recurved near the base, not twisted, with adpressed hairs up to $0.1 \mathrm{~mm}$ long. Paleas (3.7-)4-4.5(-4.8) $\mathrm{mm}$ long $\times 1.3-2(-2.5) \mathrm{mm}$ wide [ratio of palea length/lemma length $=(0.49-) 0.55-0.64(-0.73)]$, elliptic, rarely narrowly elliptic, margins with hairs from the middle till the upper part; teeth $0.1-0.4(-0.8) \mathrm{mm}$ long, densely hairy, with hairs up to $0.2 \mathrm{~mm}$ long. Lodicules (0.4-)0.6-0.8(-0.9) mm long, with 2(-3) lobules of the same or different length, sometimes slightly denticulate. Anthers (0.6-)1-1.3(-1.6) mm long. Ovary (0.3-) $0.8-1.6(-2.2) \mathrm{mm}$ long. Caryopsis (2.4-)2.6-3.3(-3.5) $\mathrm{mm}$ long $\times(0.2-) 0.3-0.5 \mathrm{~mm}$ wide, narrowly elliptic, sometimes linear. Figure 6.

Chromosome Number-2n = 28 (Ono and Tateoka 1953; Tateoka 1978), 42 (Hsu 1972).

Phenology-Flowering and fruiting from April to August.

Distribution and Habitat-This species is distributed from eastern China (Sichuan and Yunnan) to South Korea, Japan, and Taiwan, and also in New Guinea; 0-3,225 m of elevation; in moist roadsides and meadows, well-drained slopes, and shady forests. Figure 7.

Specimens Examined-CHINA. Guangdong: Tsengshing distr., Naam

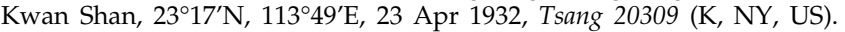

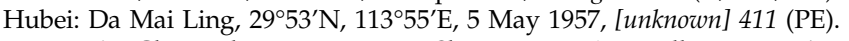
Hunan: Yi Chang distr., P'ing T'ou Shan, T'ang Wan village, $26^{\circ} 23^{\prime} \mathrm{N}$, 11050'E, May 1934, Tsang 23765 (C, G, GH, LE, PH, PR, US). Jiangsu: Nanking, Spirits Valley, 32 ${ }^{\circ} 3^{\prime} \mathrm{N}, 118^{\circ} 46^{\prime} \mathrm{E}, 11$ Jun 1929, Keng 2314 (US); Tangchuan, 32 $2^{\circ}{ }^{\prime} \mathrm{N}, 118^{\circ} 30^{\prime} \mathrm{E}, 6$ May 1922, Steward 2516 (US). Sichuan: Pujiang Xian, Ganxi, Jikongdong, $30^{\circ} 15^{\prime} \mathrm{N}, 103^{\circ} 21^{\prime} \mathrm{E}, 25$ Apr 1986, Naito 15 \& al. (PE). Yunnan: Long-Ky, $23^{\circ} 37^{\prime} \mathrm{N}, 104^{\circ} 43^{\prime} \mathrm{E}$, Jul 1911, Maire s. $n$. (AAH, NY); Hsi-lung-tang, Cheng-kiang, $24^{\circ} 39^{\prime} \mathrm{N}, 102^{\circ} 56^{\prime} \mathrm{E}, 1939$, Tsiang 16458 \& Wang (AAH). Zhejiang: Hangchow, jiu xi shi ba jian kou, $30^{\circ} 17^{\prime} \mathrm{N}, 120^{\circ} 9^{\prime} \mathrm{E}, 3$ May 1935, Keng 2977 (PE); on the road of Lao Dian to 


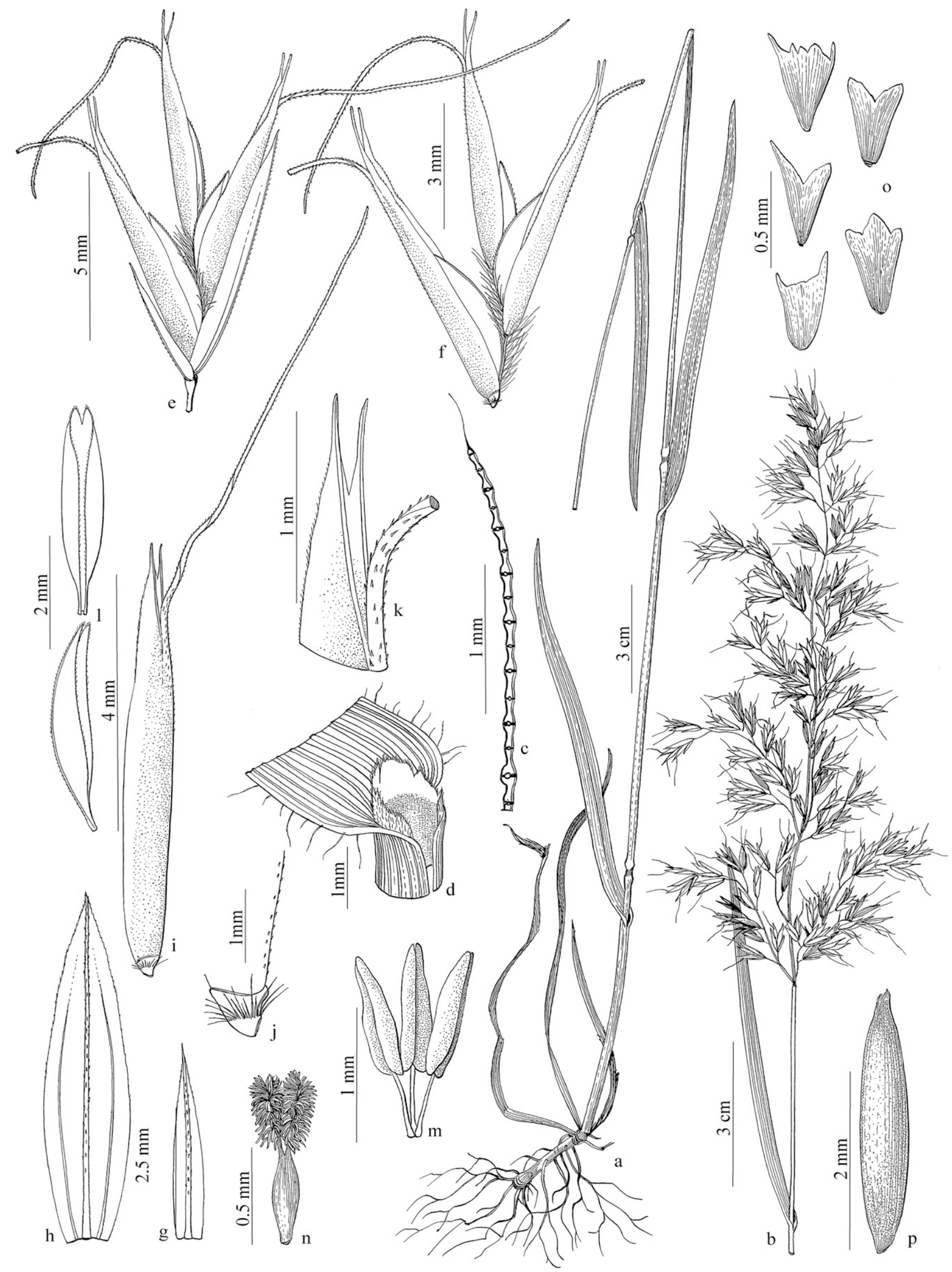

Fig. 6. Trisetum bifidum. a. Basal habit. b. Inflorescence. c. Transverse section of leaf-blade. d. Sheath, ligule, and portion of the blade. e. Spikelet. f. Florets. g. Lower glume, dorsal view. h. Upper glume, dorsal view. i. Floret. j. Callus, lateral view. k. Lemma, upper part, lateral view. 1. Palea, ventral and lateral view. m. Stamens. n. Pistil. o. Lodicules. p. Caryopsis. (Ohwi 2326, US-1647265, a-b, o-p; Koponen 15299, H, c; Keng 2977, PE-718646, d-o). 


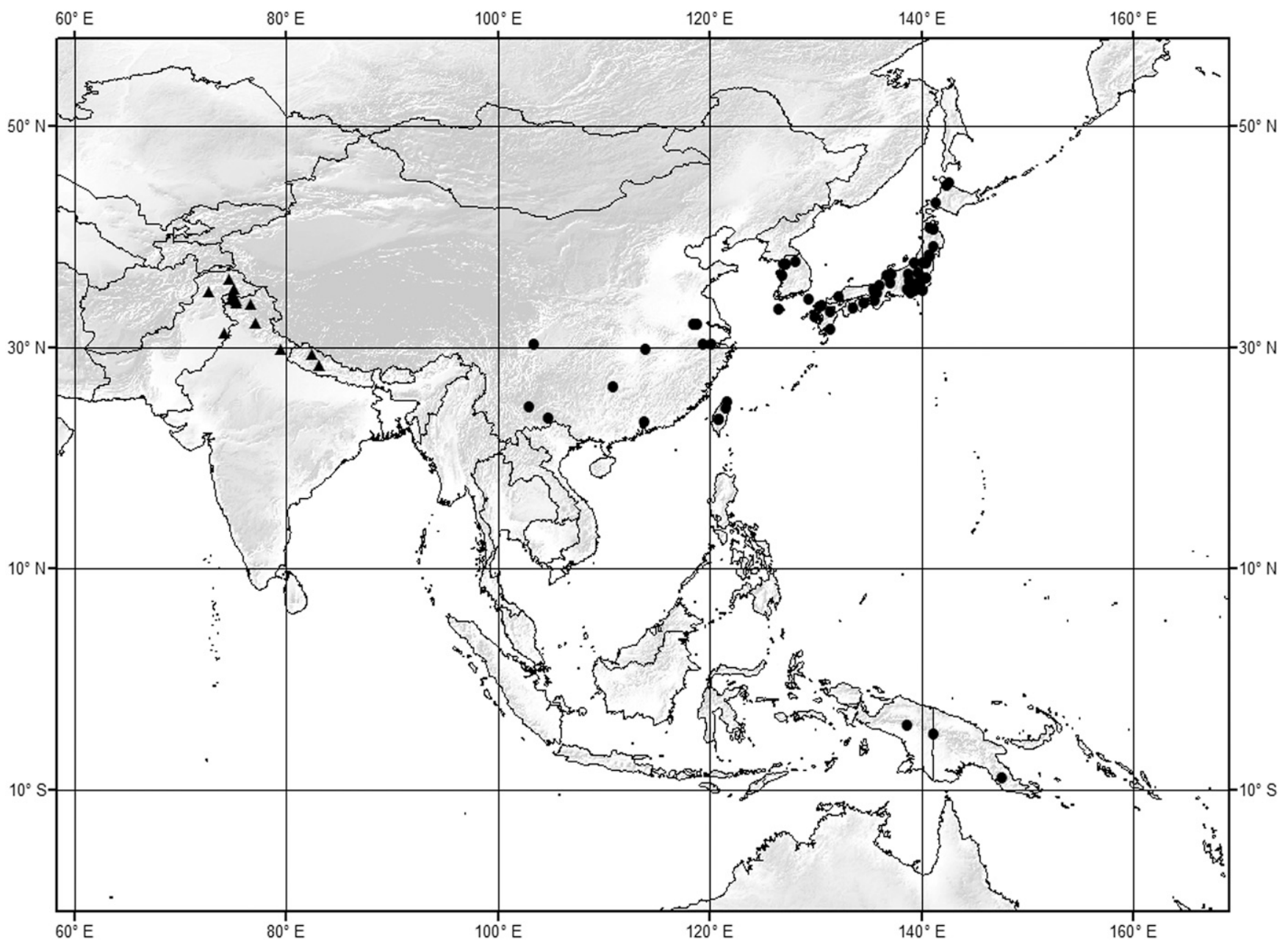

Fig. 7. Distribution of Trisetum bifidum (dots) and T. aeneum (triangles).

Xi Mao Ling, west Tian Mu Mt., 30²0'N, 119 24'E, 22 May 1957, Huang Deng 4031 (PE).

INDONESIA. Western New Guinea: Lake Habbema, $4^{\circ} 8^{\prime} \mathrm{S}, 138^{\circ} 40^{\prime} \mathrm{E}$ Aug 1938, Brass 9118 (AAH, L, US).

JAPAN. Hokkaido Island: Hokkaido pref., collines d'Yesashi, $44^{\circ} 56^{\prime} \mathrm{N}$, 142 ${ }^{\circ} 35^{\prime}$ E, 22 Jul 1890, Faurie 5711 (G, P); Hokkaido pref., environs de

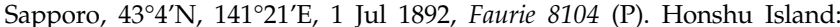
Shizuoka pref., Izu Peninsula, Shuzenji, $34^{\circ} 58^{\prime} \mathrm{N}, 138^{\circ} 55^{\prime} \mathrm{E}, 12$ May 1929 , Beattie 10622 \& Kurihara (US); Wakayama pref., Koya-san, $34^{\circ} 13^{\prime} \mathrm{N}$, $135^{\circ} 35^{\prime}$ E, 23 Jun 1929, Beattie 10832 \& Kurihara (US); Kyoto pref., Kyoto city, Sakyo-ku, Kyoto University campus, southeast of the intersection of Imadegawa-dori and Higashiyo jidori (Hyaku-man-ben), $35^{\circ} 15^{\prime} \mathrm{N}$, $135^{\circ} 26^{\prime} \mathrm{E}, 15$ May 1977, Boufford 18987a \& Wood (AAH, MO); Kanagawa pref., Chigasaki, $35^{\circ} 20^{\prime} \mathrm{N}, 139^{\circ} 24^{\prime} \mathrm{E}$, 15 May 1954, Dahlstrand s. $n$. (GOET); Tokyo pref., near Kichijoji, $35^{\circ} 42^{\prime}$ N, $139^{\circ} 34^{\prime}$ E, 12 Jun 1929, Dorsett 483 \&

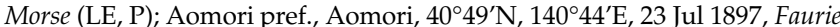
1212 (WU); lbaraki pref., $36^{\circ} 17^{\prime} \mathrm{N}, 140^{\circ} 25^{\prime} \mathrm{E}$, May 1900, Faurie 4469 (US); Niigata pref., environs de Shirosaki, $37^{\circ} 42^{\prime} \mathrm{N}, 139^{\circ} 22^{\prime} \mathrm{E}, 29$ May 1886, Faurie

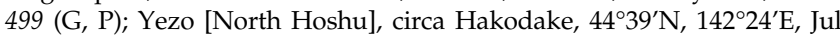
1902, Faurie 5214 (BM); Aomori pref., plaine de Shichinohe, $40^{\circ} 41^{\prime} \mathrm{N}$, $141^{\circ} 9^{\prime} \mathrm{E}, 17$ Jun 1886, Faurie 689 (FI, P); Kanagawa pref., Yokoska [Yokosuka], $35^{\circ} 17^{\prime} \mathrm{N}$, $139^{\circ} 40^{\prime} \mathrm{E}, 1866-71$, Franchel 1472 (LE, P, W); Tochigi pref., foot of Mt. Kogashi-yama, Ashigaru, Utsunomiya-shi, $36^{\circ} 37^{\prime} \mathrm{N}, 139^{\circ} 46^{\prime} \mathrm{E}$,

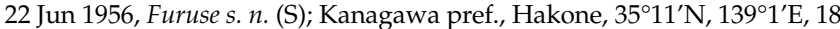
Jul 1921, Hitchcock 18318 (US); Tochigi, Nikko to Chuzenji Lake, $36^{\circ} 45^{\prime} \mathrm{N}$, 139 $37^{\prime} \mathrm{E}, 24 \mathrm{Jul}$ 1921, Hitchcock 18344 (US); Mizusawa-shi, in Rikuchu, $39^{\circ} 7^{\prime} \mathrm{N}, 141^{\circ} 10^{\prime} \mathrm{E}, 19 \mathrm{Jun} 1938$, Iwabuchi s. n. (US); within the town limits of Yatsuo, $17 \mathrm{~km}$ south of the city of Toyama, $36^{\circ} 35^{\prime} \mathrm{N}$, $137^{\circ} 7^{\prime} \mathrm{E}, 8$ Jun 1955 , Kirino 652 (US); Tohoku distr., Miyagi pref., Sendai-shi, Aoba-ku, Aoshita, $38^{\circ} 18^{\prime} \mathrm{N}, 140^{\circ} 38^{\prime} \mathrm{E}$, 10 Jun 1994, Kondo 227 \& Kurosawa (AAH); Shizuoka pref., Higashitanaka, Gotenba city, E foot of Mt. Fuji, $35^{\circ} 19^{\prime} \mathrm{N}, 138^{\circ} 56^{\prime} \mathrm{E}, 25$ May 1977, Konta 1184 \& Murata (AAH); Miyagi pref., Tohoku distr.,
Sendai-shi, S foot of Mt. Izumigatake, Yoshinodaira Bog, $38^{\circ} 23^{\prime} \mathrm{N}$, $140^{\circ} 44^{\prime} \mathrm{E}, 11$ Jul 1992, Kurosawa 5063 (AAH); Fukshima pref., Tohoku distr., Fukushima-shi, in the campus of Fukushima University, $37^{\circ} 40^{\prime} \mathrm{N}$, $140^{\circ} 28^{\prime} \mathrm{E}, 18$ Jun 1997, Kurosawa 10102 \& al. (AAH); Chiba pref., Ichikawa, Kônodai, $35^{\circ} 43^{\prime} \mathrm{N}, 1^{\circ} 9^{\circ} 55^{\prime} \mathrm{E}, 29$ May 1898, Makino s. n. (AAH, LE); Musashi prov., Sekido, $35^{\circ} 15^{\prime} \mathrm{N}, 139^{\circ} 36^{\prime} \mathrm{E}, 3$ Jun 1946, Mizushima s. n. (S); Shizuoka pref., Gendouji, Fujinomiya city, SW foot of Mt. Fujji, $35^{\circ} 13^{\prime} \mathrm{N}$, 138³7'E, 21 Apr 1977, Murata 11739 \& al. (AAH); Gunma pref., Tanogun, Nakazato-mura, Mt. Kanoosan, 36² $4^{\prime} \mathrm{N}, 138^{\circ} 50^{\prime} \mathrm{E}, 29$ May 1976, Murata 1821 \& al. (AAH); Tokyo pref., Shinagawa-ku, Oifuto, $35^{\circ} 36^{\prime} \mathrm{N}$, $139^{\circ} 43^{\prime}$ E, 17 Jun 1984, Nakamura s. n. (M); Chiba pref., Mt. Kiyosumi, Amatsukominato-machi, Awa-gun, $35^{\circ} 9^{\prime} \mathrm{N}, 140^{\circ} 9^{\prime} \mathrm{E}, 9$ May 1988 , Ohba s. n. \& al. (AAH); Kyoto pref., Ogura, prope Kyoto, 34 ${ }^{\circ} 53^{\prime} \mathrm{N}, 135^{\circ} 46^{\prime} \mathrm{E}, 16$ May 1931 , Ohwi 58 (B, GOET, H, LE, UPS); Tsuruga, $35^{\circ} 38^{\prime} \mathrm{N}, 136^{\circ} 3^{\prime} \mathrm{E}, 27$ May 1928,

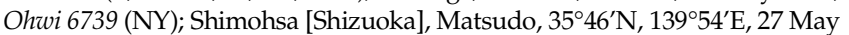
1926, Ohwi s. n. (NY); Gunma pref., Shima Tone-gun, along the Shinyu river, $36^{\circ} 41^{\prime} \mathrm{N}, 138^{\circ} 46^{\prime} \mathrm{E}$, 20 Jun 1964, Ono s. n. \& Kobayashi (LE, US); Hirosima pref., Sandankyo, $34^{\circ} 37^{\prime} \mathrm{N}, 132^{\circ} 11^{\prime} \mathrm{E}$, 3 Jun 1933 , Sato s. $n$. (US); Hoshu, $35^{\circ} 47^{\prime} \mathrm{N}$,

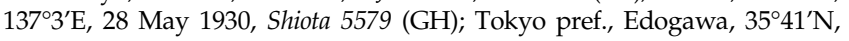
$139^{\circ} 52^{\prime}$ E, 22 May 1955, Tanaka s. n. (K); Kanagawa pref., Hakone, $35^{\circ} 11^{\prime} \mathrm{N}$,

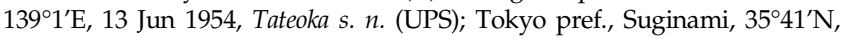
$139^{\circ} 38^{\prime}$ E, 15 May 1952, Tateoka s. n. (B, C, UPS); Kyoto pref., Ikejiri, Umaji-cho, Kameoka-shi, 350N, 135³2'E, 22 May 1997, Tsugaru 24822 \& Takahashi (AAH); Kyoto pref., $\mathrm{N}$ foot of the Mt. Ponpon-yama, Oharanoishidzukuri-cho, Kyoto-shi, 3456'N, 135³7'E, 6 Jun 1997, Tsugaru 25044 \& Takahashi (AAH); Fukushima pref., Bandai, $37^{\circ} 36^{\prime} \mathrm{N}, 140^{\circ} 4^{\prime}$ E, 9 Jul 1918, Yasuda s. n. (L); Saitama

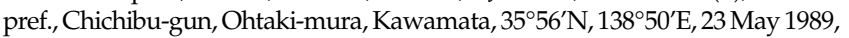
Yokota 958 (AAH); Miyagi pref., Sendai-shi, Aoba-ku, Aramaki, Nitayachi, $38^{\circ} 16^{\prime} \mathrm{N}, 140^{\circ} 49^{\prime} \mathrm{E}$, 15 Jun 1992, Yonekura 448 (AAH). Kyushu Island: Oita pref., Mt. Yufu, $33^{\circ} 16^{\prime} \mathrm{N}, 131^{\circ} 23^{\prime} \mathrm{E}, 12$ May 1928, Kondo s. n. (B); Miyazaki pref., Nichinan-shi, Kamishirakimata, valley of Sakatanigawa, $31^{\circ} 38^{\prime} \mathrm{N}, 131^{\circ} 20^{\prime} \mathrm{E}$,

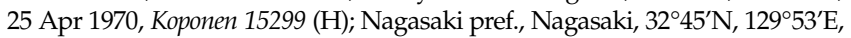


1863, Maximowicz s. n. (BM, P); Fukuoka pref., Kasuya, Tatara, Najima, $33^{\circ} 38^{\prime} \mathrm{N}, 130^{\circ} 25^{\prime} \mathrm{E}, 14 \mathrm{Jul}$ 1925, Schikama 209 (PH); Fukuoka pref., Katsukimura, Ongagun, $33^{\circ} 51^{\prime} \mathrm{N}, 130^{\circ} 38^{\prime} \mathrm{E}$, 5 Jun 1930, Takenouchi s. n. (B). Shikoku Island: Kochi pref., prope Tokushima, $34^{\circ} 4^{\prime} \mathrm{N}, 134^{\circ} 34^{\prime} \mathrm{E}$, Jun 1900, Faurie 4468 (US); Tokushima pref., Inakochimura in Nanishigun, $34^{\circ} 4^{\prime} \mathrm{N}$,

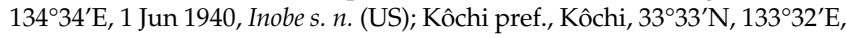
May 1893, Makino s. n. (AAH). Tsushima island: $34^{\circ} 20^{\prime} \mathrm{N}, 129^{\circ} 20^{\prime} \mathrm{E}, 1859$, Wilford 938 (GH, K)

PAPUA NEW GUINEA. Western Province: Mount Victoria area Iswan Swamp, c. $2 \mathrm{~km}$ SSW of Mount Service, on bank of Koma Creek, 855'S, 147³2'E, 19 May 1976, van Royen 10867 (L). Hela: West Sepik, Star Mts., Camp 1, Tel Basin, 50'S, 141 ${ }^{\circ} 5^{\prime} \mathrm{E}, 2$ May 1975, Veldkamp 6248 (L).

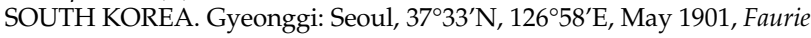

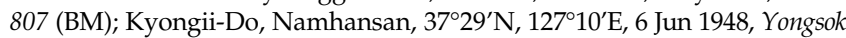

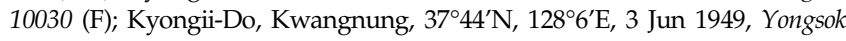

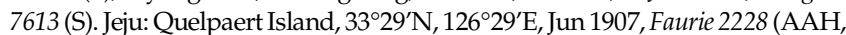
BM, G, LE, W); Quelpaert Island, Hallasan, 3321'N, $126^{\circ} 31^{\prime} \mathrm{E}$, Jul 1909, Taquet 3423 (AAH, NY); Quelpaert Island, Yengsil [Yeongsil], 36³2’ N, 126 51'E, Aug 1911, Taquet 5096 (AAH, NY).

TAIWAN. Chiayi Co., A-li-shan, $23^{\circ} 30^{\prime} \mathrm{N}, 120^{\circ} 48^{\prime} \mathrm{E}, 19$ May 1969 , Chienchang Hsu 5394 \& Kuo (S); 17 Aug 1957, Ream 589 (US); Chiayi Co., A-li-shan to Tungpu, $23^{\circ} 30^{\prime} \mathrm{N}, 120^{\circ} 48^{\prime} \mathrm{E}$, 19 Jun 1970 , Chien-chang Hsu 7293 \& Kuo (G);

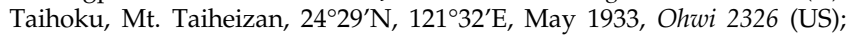
Taipei, prope Pianan-anbu, $25^{\circ} 2^{\prime} \mathrm{N}, 121^{\circ} 38^{\prime} \mathrm{E}$, Jun 1933, Ohwi 2720 (UPS, US).

Notes-Trisetum bifidum has a disjunct range, occurring in the continental part of Asia and Taiwan, and in the mountains of New Guinea. This particular distribution happens also in other species, as indicated by Veldkamp and van der Have (1983).

Chrtek (1968) noted that T. bifidum is closely related to the species from $T$. sect. Trisetum, differentiated mainly by its palea with longer and sharpest hairs on the keels than $T$. sect. Trisetum, which has shorter ones. As Veldkamp and van der Have (1983) said, it has been considered that this species is closely related to $T$. flavescens (generic type of $T$. ser. Trisetum), being very close to $T$. sibiricum. Consequently, and because of its characters, these authors included T. bifidum in the series Sibirica following Chrtek (1968) or in subsection Sibirica according to Probatova (1978). Both of the species, T. bifidum and T. sibiricum, share part of their distribution, but they can be easily differentiated. Trisetum bifidum is separated from the other species of $T$. sect. Sibirica, and more specifically of $T$. sibiricum, mainly by having a palea about half the length of the lemma [ratio of palea length/lemma length $=(0.49-) 0.55-0.64(-0.73)]$, the awn inserted more apically [ratio of awn insertion from the base length/lemma length $=(0.56-) 0.62-0.74(-0.83)]$, and longer callus indumentum (0.3-0.7 mm long).

4. Trisetum henryi Rendle, J. Linn. Soc., Bot. 36: 400. 1904.TYPE: CHINA. Hubei, Fang Xian, $32^{\circ} 05^{\prime} \mathrm{N} 110^{\circ} 35^{\prime} \mathrm{E}$, 1885-1888, A. Henry 6643 (lectotype, here designated: K-808693!; isolectotypes: B-10_0279997!, BM-959387!, GH-24560!).

Herb (110-)121-150(-165) cm high, shortly rhizomatous, culm 1.5-4 mm diam, glabrous; nodes (6-)7-10(-12), separated along the culm, enfolded or not by the sheaths, glabrous. Basal leaf-sheaths glabrous, sometimes very slightly pubescent, with hairs less than $0.1 \mathrm{~mm}$ long, decaying into fibers, yellowish to brownish; basal leaf-blades 14-18(-20) $\mathrm{mm}$ long $\times$ 6-8 mm wide, flat, rolled when dried, glabrous abaxially and adaxially, usually with long hairs on the nerves abaxially, sometimes with hairs on the margins $0.05-1 \mathrm{~mm}$ long, brownish to dark green; top culm leaf-sheaths (12.5-) 17-23(-25) cm long, longer than the internodes, involving most of the culm, glabrous, normally pubescent on the upper part, with cilia on the margins; top culm leaf-blades 17.5-29.5 cm long $\times(5.2-) 6-8(-10) \mathrm{mm}$ wide, flat, not envolving the culm and extending to the panicle, with the central nerve conspicuously marked, with hairs abaxially along the nerves and margins, glabrous to slightly pubescent adaxially, with hairs, also along the nerves, dark green to brownish; inner collar region pubescent, also on the margins, with hairs (0.1-) 0.8-1 mm long; ligules (1.5-)2-2.8(-3.4) mm long, laciniate or irregular dentate, pubescent to slightly pubescent abaxially, with hairs $0.1-1(-1.4) \mathrm{mm}$ long. Basal node of the panicle glabrous. Panicles (16-)19-23(-28) cm long $\times(4-) 5-8.5 \mathrm{~cm}$ wide, elliptic to narrowly elliptic-oblong in outline, lax to slightly dense; rachis glabrous; branches in whorls of 3 to 7 , longest basal branches (4.4-)5.8-9(-11) cm long. Spikelets (5.2-) 6-7.2(-8) mm long $\times(2-) 2.3-3.7(-5.6) \mathrm{mm}$ wide, 2-3-flowered; pedicels (2-)3.3-5(-6.8) $\mathrm{mm}$ long, glabrous, rarely with hairs up to $0.05 \mathrm{~mm}$ long. Glumes unequal [ratio of lower glume length/upper glume length $=0.6-0.73(-0.76)]$; lower glume (2.8-)3.2-4.5 mm long $\times 0.6-0.8 \mathrm{~mm}$ wide, narrowly lanceolate [ratio of lower glume width/lower glume length = $0.17-0.21(-0.25)]$, acuminate, sometimes acute, 1-nerved, glabrous, with hairs up to $0.05 \mathrm{~mm}$ long from the middle to the upper part of the midrib, greenish to yellowish; upper glume 4.6-6.2 mm long $\times 1.4-1.6 \mathrm{~mm}$ wide, narrowly elliptic to oblanceolate (ratio of upper glume width/upper glume length $=0.23-0.3$ ), acuminate, rarely acute, 3-nerved, glabrous, with hairs up to $0.05 \mathrm{~mm}$ long from the middle to the upper part of the central nerve, greenish to yellowish, sometimes brownish; rachilla segments between first and second floret 1.1-1.7 $\mathrm{mm}$ long, with hairs (0.5-)0.7-1 $(-1.2) \mathrm{mm}$ long; rachilla segments to sterile floret 1.1-1.4 mm long, with hairs $0.4-0.6(-0.8) \mathrm{mm}$ long. Lemmas (4.2-) 4.8-6.1 mm long $\times 0.6-0.8 \mathrm{~mm}$ wide, narrowly to broadly lanceolate [ratio of lemma width/lemma length $=0.21-0.28$ $(-0.36)]$, scabridulous, with hairs on the midrib up to $0.05 \mathrm{~mm}$ long, pale yellowish or brownish, with broad hyaline margins; apical teeth (0.05-)0.1-0.4 mm long, without or with aristules 0.1-0.45 mm long; awn (4.3-)4.7-6.5(-6.8) $\mathrm{mm}$ long, inserted 2.5-3.5(-4) $\mathrm{mm}$ from the base [ratio of awn insertion from the base length/lemma length $=0.55-0.60(-0.66)]$, recurved and not twisted or sometimes slightly twisted at the base, with adpressed hairs up to $0.05 \mathrm{~mm}$ long; callus $0.2-0.3 \mathrm{~mm}$ long, orbicular to elliptic, glabrous. Paleas (3.6-)4 $-4.5 \mathrm{~mm}$ long $\times 1.2-1.5(-1.7) \mathrm{mm}$ wide [ratio of palea length/ lemma length $=0.67-0.82(-0.86)]$, elliptic, margins with hairs from the middle till the upper part; teeth (0.2-)0.3-0.4 $(-0.6) \mathrm{mm}$ long, with short antrorse hairs. Lodicules 0.4-0.8 mm long, with 2 lobules of the same length. Anthers (1.5-)1.8-2.4 mm long. Ovary (0.5-)0.8-1.3 mm long. Caryopsis $3-3.4 \mathrm{~mm}$ long $\times 0.3-0.6 \mathrm{~mm}$ wide, oblanceolate. Figure 8.

Chromosome Number-Unknown.

Phenology-Flowering and fruiting from February to September.

Distribution and Habitat-Endemic from eastern and central China; 1,000-2,400 m of elevation; at open meadows, shady forests. Figure 9.

Specimens Examined-CHINA. Anhui: Li Shan, NW Chemen, $30^{\circ} 45^{\prime} \mathrm{N}$, $119^{\circ} 20^{\prime} \mathrm{E}$, Aug 1925, Ching 8767 (US). Henan: Huopenjin, Lushi, Dalindi, $33^{\circ} 58^{\prime} \mathrm{N}, 110^{\circ} 59^{\prime} \mathrm{E}, 7 \mathrm{Jul}$ 1959, [unknown] 34437 (PE); Chimanmeizijian, Haoxian, $34^{\circ} 21^{\prime} \mathrm{N}, 111^{\circ} 58^{\prime} \mathrm{E}, 17$ Aug 1959, [unknown] 34986 (PE); Funyu Shan, Xiangshuitai, Tongbaitaohuadong, $32^{\circ} 22^{\prime} \mathrm{N}, 113^{\circ} 23^{\prime} \mathrm{E}, 15$ Aug 1956, Forestry Administration Henan 465 (PE); Laoxiang-ling, Huangshi Xiang, 


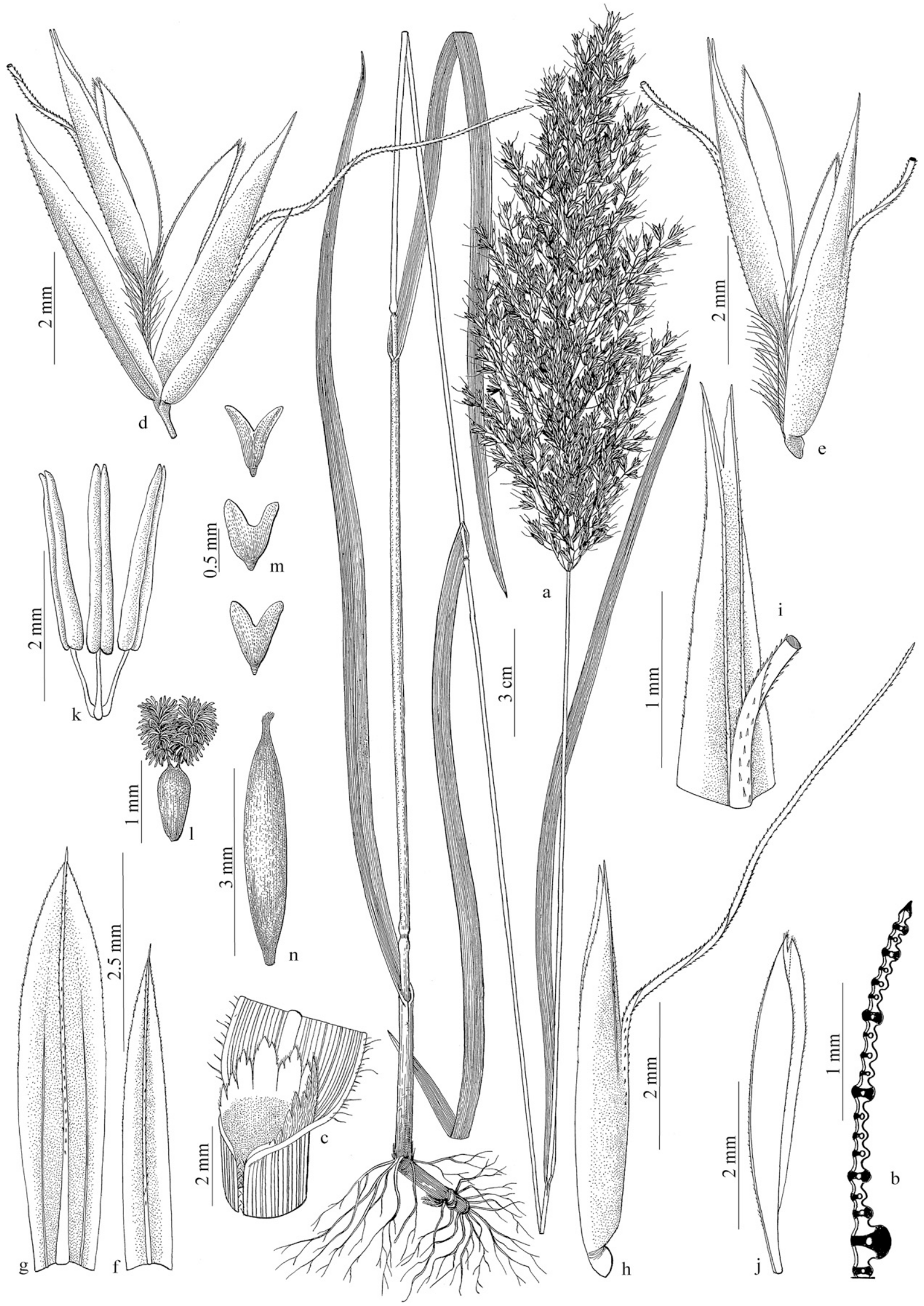

FIg. 8. Trisetum henryi. a. Habit. b. Transverse section of leaf-blade. c. Sheath, ligule, and portion of the blade. d. Spikelet. e. Florets. f. Lower glume, dorsal view. g. Upper glume, dorsal view. h. Floret. i. Lemma, upper part, lateral view. j. Palea, lateral view. k. Stamens. 1. Pistil. m. Lodicules. n. Caryopsis. (Henry 6643, K-808693, a, m; Ching 8767, US, b; Steward 2559, US-1346322, c-j; [unknown] 34986, PE-718805, k-n). 


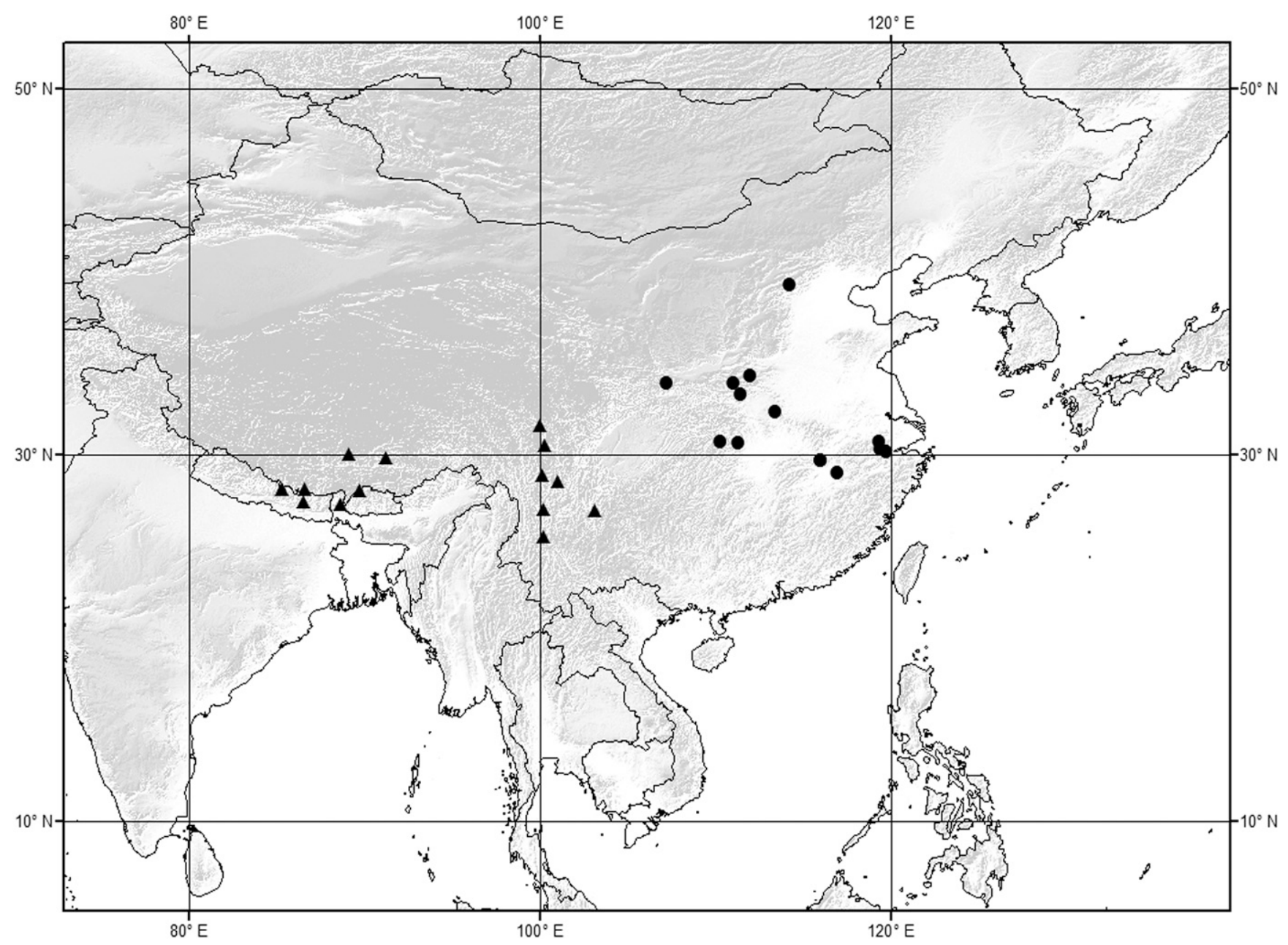

FIG. 9. Distribution of Trisetum henryi (dots) and T. scitulum (triangles).

Xixia, 3320'N, 111²5'E, 12 Jul 1960, Henan Team 1023 (PE); 17 Jul 1960,

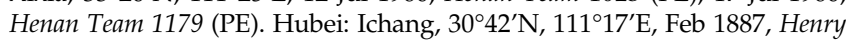
2558 (LE, P); Mars 1889, Henry 6706 (K, US). Jiangxi: Lu Shan, 29³’N, $116^{\circ} 57^{\prime} \mathrm{E}, 10$ Aug 1922, Steward 2559 (C, G, K, LE, MO, NY, US); Kuling, mountains south of Kiukiang, $2^{\circ} 42^{\prime} \mathrm{N}, 116^{\circ} 0^{\prime} \mathrm{E}, 16$ Aug 1921, Hitchcock 18534 (US), 18551 (US); 17 Aug 1921, Hitchcock 18558 (US). Shaanxi: Taibai County, Baiyun, $33^{\circ} 58^{\prime} \mathrm{N}, 107^{\circ} 12^{\prime} \mathrm{E}, 11 \mathrm{Jul} 1938$, Liou \& al. 2042 [PE-718810 (image!)]. Zhejiang: Changhua, Lin'an city, $30^{\circ} 14^{\prime} \mathrm{N}, 119^{\circ} 43^{\prime} \mathrm{E}, 13$ Aug 1957, He Yinyu 25309 [HHBG-HZ38395 (image!)].

Notes-Rendle studied the grasses of China and its neighboring regions in the article published by Forbes and Hemsley (1904). In this study, he described T. henryi as closely allied to T. sibiricum (identified as T. flavescens), clearly differentiated by being taller and more robust, with more dense panicles and silvery-brown spikelets, and lower glume less acute. Apart from those characters, this species is also characterized by having longer panicles, with longer branches than T. sibiricum, always long leaves, the upper one usually exceeding the panicle, with marked central nerves, and longer ligules. It has also spikelets with broad hyaline margins, and lemmas with shorter awns than T. sibiricum subsp. sibiricum.

One of the studied specimens from the mountains of Zhejiang [Yao Kan 79429 (MO-3690121)] is exceptional by its smaller panicle and spikelets $(11 \mathrm{~cm}$ long, and up to $5 \mathrm{~mm}$ long, respectively). However, it has a thick culm and long leaves with a marked central nerve, as in T. henryi.
Wu and Phillips (2006), in the Flora of China, noted that $T$. henryi is also present in the provinces Jiangsu, Shanxi and South-Eastern Sichuan, from where we have not seen material, but it is surely present there.

5. Trisetum aeneum (Hook. f.) R. R. Stewart, Brittonia 5: 431. 1945. Trisetum aureum Nees ex Steud., Syn. Pl. Glumac. 1: 225. 1854, nom. illeg., non Ten. 1820. Avena aenea Hook. f., Fl. Brit. India 7: 279. 1896. Trisetum sibiricum subsp. aenea (Hook. f.) Roshev., Izv. Glavn. Bot. Sada R.S.F.S.R. 21: 91. 1922.-TYPE: INDIA. Himachal Pradesh, Shimla distr., Buran Pass, Kedarkanta, 312’N 78 ${ }^{\circ}$ 'E, J. F. Royle 94 (lectotype, here designated: LIV-1952.121.10949 (image!); isolectotypes: LIV-1952.121.10948 (image!), LIV-1952.121.10950 (image!)).

Herb (45-)53-70 cm high, not or loosely tufted, shortly rhizomatous, culm $0.8-1.2(-2) \mathrm{mm}$ diam, glabrous; nodes (3-)4-6, separated along the culms, not enfolded by the sheaths, glabrous. Basal leaf-sheaths glabrous, rarely shortly pubescent, with hairs up to $0.08 \mathrm{~mm}$ long, decaying into fibers, brownish to yellowish, sometimes greenish; basal leafblades 6-12(-13.5) cm long $\times(2.3-) 2.8-3.8(-4) \mathrm{mm}$ wide, flat, rolled when dried, pubescent with longer hairs abaxially, glabrous to slightly pubescent adaxially, margins with hairs (0.05-)0.1-0.8 mm long, yellowish to brownish, sometimes 
greenish; top culm leaf-sheaths (10-)12-18.5 cm long, shorter than the internodes, glabrous; top culm leaf-blades (7-) 8.5-14.5(-16) $\mathrm{cm}$ long $\times 4-5.6 \mathrm{~mm}$ wide, flat, sometimes parallel to the culm and extending to the panicle, with the central nerve similar to the lateral ones, glabrous abaxially and pubescent adaxially, with adpressed hairs along the nerves on both sides, longer adaxially, with hairs on the margins (0.05-)0.1-1(-1.3) mm long, greenish to greyish, sometimes brownish; inner collar region glabrous, with hairs on the margins $0.4-1 \mathrm{~mm}$ long; ligules (1-)1.3-3 mm long, laciniate, rarely denticulate, glabrous, rarely with scattered hairs on the apex (0.05-)0.1-0.2(-0.4) mm long. Basal node of the panicle glabrous. Panicles (5.7-)7-12.5(-15) $\mathrm{cm}$ long $\times$ $1.4-2.5(-3) \mathrm{cm}$ wide, oblong to elliptic in outline, rarely oblanceolate, dense, with the rachis visible in some parts; rachis glabrous; branches in whorls of 3 or 4 , longest basal branches (0.7-)0.8-1.7(-3.1) cm long. Spikelets 6-7.5 mm long $\times(2-)$ 2.4-3.7(-4.3) mm wide, 2-3-flowered; pedicels 2-4(-5) $\mathrm{mm}$ long, slightly pubescent, with hairs up to $0.08 \mathrm{~mm}$ long. Glumes subequal [ratio of lower glume length/upper glume length $=(0.8-) 0.82-0.89(-0.95)]$; lower glume $(4.5-) 4.8-5.5$ (-6) $\mathrm{mm}$ long $\times 1-1.2(-1.4) \mathrm{mm}$ wide, narrowly lanceolate, rarely broadly lanceolate (ratio of lower glume width/ lower glume length $=0.2-0.24$ ), acuminate, rarely long acuminate, 1-nerved, glabrous, with hairs up to $0.1 \mathrm{~mm}$ long on the upper part of the nerve and margins, brownish to yellowish, sometimes greenish; upper glume $5.4-6.3 \mathrm{~mm}$ long $\times 1.7-2 \mathrm{~mm}$ wide, narrowly elliptic to oblong, sometimes narrowly to broadly lanceolate [ratio of upper glume width/upper glume length $=(0.27-) 0.29-0.34$ $(-0.36)]$, acuminate, rarely long acuminate, 3-nerved, glabrous, with hairs up to $0.1 \mathrm{~mm}$ long, from the middle to the upper part of the central nerve and sometimes on the upper part of the margins, brownish to yellowish, sometimes greenish on the central part; rachilla segments between first and second floret $1-1.8 \mathrm{~mm}$ long, with hairs $0.7-1.4 \mathrm{~mm}$ long; rachilla segments to sterile floret $0.8-1.7 \mathrm{~mm}$ long, with hairs 0.1-0.7 mm long. Lemma (5-)5.6-6(-7.8) mm long $\times 1-1.2$ $(-1.4) \mathrm{mm}$ wide, oblong to broadly lanceolate [ratio of lemma width/lemma length $=(0.24-) 0.25-0.32(-0.36)]$, scabridulous, with hairs on the midrib up to $0.1 \mathrm{~mm}$ long, brownish to yellowish, rarely greenish; callus $0.3-0.4 \mathrm{~mm}$ long, elliptic to oblong, with hairs $0.1-0.3 \mathrm{~mm}$ long; apical teeth $0.1-1.1$ $(-1.5) \mathrm{mm}$ long, with aristules $0.3-0.5(-0.7) \mathrm{mm}$ long; awn 6.5-8(-8.7) $\mathrm{mm}$ long, inserted $3-3.3(-5) \mathrm{mm}$ from the base (ratio of awn insertion from the base length/lemma length $=$ 0.5-0.64), recurved at the base, not or very slightly twisted, with adpressed hairs up to $0.05 \mathrm{~mm}$ long. Paleas (3.6-) $4.2-4.6 \mathrm{~mm}$ long $\times 1.5-1.8 \mathrm{~mm}$ wide [ratio of palea length/ lemma length $=(0.61-) 0.7-0.82)]$, elliptic, sometimes narrowly elliptic, margins with short antrorse hairs; teeth $0.1-0.5 \mathrm{~mm}$ long, with short antrorse hairs from the middle to the upper part. Lodicules $0.6-0.7 \mathrm{~mm}$ long, with 2-3 irregular lobules, rarely laciniate. Anthers (0.8-)1-1.2(-1.3) mm long. Ovary 0.6-1.3 mm long. Caryopsis $2.7-3.7 \mathrm{~mm}$ long $\times 0.5-0.8 \mathrm{~mm}$ wide, oblong to narrowly elliptic. Figure 10.

Chromosome Number-Unknown.

Phenology-Flowering and fruiting from July to September.

Distribution and Habitat-It is distributed from north Pakistan and India to central Nepal, at 3,000-4,200 m of elevation, in alpine meadows and grasslands. Figure 7.

Specimens Examined-INDIA. Himachal Pradesh: Manali, $32^{\circ} 15^{\prime} \mathrm{N}$

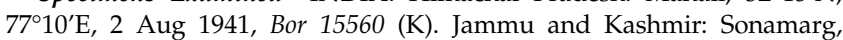

Tajivaz, $34^{\circ} 18^{\prime} \mathrm{N}, 7^{\circ} 17^{\prime} \mathrm{E}$, 18 Aug 1893, Duthie 13646 (W); Kashmir, $33^{\circ} 55^{\prime} \mathrm{N}, 76^{\circ} 40^{\prime} \mathrm{E}$, Levinge 27424 (LE); Kun Patthar, Masjid Gali, $34^{\circ} 24^{\prime} \mathrm{N}$,

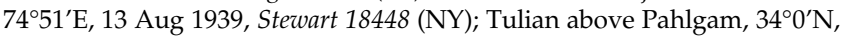
75²1'E, 30 Aug 1945, Stewart 21829 (K, NY, US). Uttarakhand: Kumaon, above Tola, $29^{\circ} 50^{\prime} \mathrm{N}, 79^{\circ} 30^{\prime} \mathrm{E}$, Strachey 1 \& Winterbottom (P); Chitona, Tehri, $30^{\circ} 23^{\prime} \mathrm{N}, 78^{\circ} 28^{\prime} \mathrm{E}$, 29 Sep 1948, Koelz 22048 (US). North-West India, Royle s. $n$. $(\mathrm{K}-808692, \mathrm{LE})$.

NEPAL. Karnali: Jumla distr., Maharigaon, 5 miles NE, $29^{\circ} 20^{\prime} \mathrm{N}$, $82^{\circ} 23^{\prime} \mathrm{E}, 22 \mathrm{Jul}$ 1952, Polunin 281 \& al. (K, UPS). Dhawalagiri: Baglung distr., near Dogadi Khola, $28^{\circ} 25^{\prime} \mathrm{N}, 8^{\circ} 10^{\prime} \mathrm{E}, 7$ Aug 1954, Stainton $3785 a$ \& al. (UPS); 29 Sep 1954, Stainton 4648 \& al. (UPS).

PAKISTAN. Azad Cachemira: Kashmir, Sind Valley, 3 Sep 1876, Clarke 31034 (K); Kamri Pass, 34²4'N, 7456'E, 18 Aug 1939, Stewart 18700 (NY, US). Gilgit-Baltistan: Kamri Valley, near Kalapani, $35^{\circ} 18^{\prime} \mathrm{N}, 75^{\circ} 7^{\prime} \mathrm{E}$, 25 Aug 1892, Duthie 12548 (W); Kalapani, Kamri Pass, $35^{\circ} 18^{\prime} \mathrm{N}, 7^{\circ} 7^{\prime} \mathrm{E}, 25$ Aug 1892, Duthie 12562 (W); Baghrot Valley, 36 $6^{\circ} \mathrm{N}, 7^{\circ} 35^{\prime} \mathrm{E}, 20$ Sep 1990, Miehe 3891 \& Miehe (K). Khyber

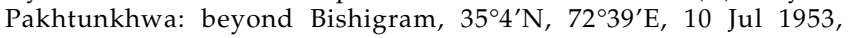
Stewart 25001 \& Rahman (K).

Notes-Trisetum aeneum was already included by Chrtek (1968) in T. ser. Sibirica, because of its basally recurved and slightly twisted awns. This species is distinguished from the rest of species by its dense and usually contracted panicles, subequal glumes, and small anthers.

In its eastern distribution, this species overlaps with the westernmost $T$. scitulum, in central Nepal; both species have a similar habitat but they are really easy to differentiate, because of their different habit, T. scitulum having lax panicles, and branches single or paired instead of being in whorls of more than three as in the rest of species of the section.

From Trisetum sibiricum subsp. sibiricum, present just north and east of the range of T. aeneum, it is mainly differentiated by anther length, panicle shape, and by its subequal glumes. Roshevitz (1922) considered Trisetum aeneum to be a subspecies of $T$. sibiricum, only taking into account its dense panicles and bright bronze spikelets. We consider T. aeneum a good species having a separate distribution, and dense panicles, and smaller stamens that are a consistent character in the species of the section.

Steudel (1854) when described Trisetum aureum mentioned Royle's collection as follows: "Royle hrbr. nr. 44. Nepal". According to G. Reid (pers. comm.), there was a misprint or a transcription error because the type material is actually Royle 94, not Royle 44. An additional fact to support this conclusion is that the specimen Royle 44 corresponds to Panicum vestitum Kunth.

6. Trisetum Scitulum Bor ex Chrtek, Acta Univ. Carol., Biol. 1967: 105. 1967.-TYPE: INDIA. Sikkim, $27^{\circ} 20^{\prime} \mathrm{N} 88^{\circ} 37^{\prime} \mathrm{E}$, J. D. Hooker s. n. (holotype: K-32269!; isotypes: GH!, K!, L-50297 (image!), P-2255859!, W-25045!).

Herb 12-80 cm high, loosely tufted, shortly rhizomatous, culm (0.4-)0.5-2 mm diam, glabrous; nodes 2-3(-4), separated along the culm, not enfolded by the sheaths, glabrous. Basal leaf-sheaths glabrous, sometimes slightly to densely pubescent, with hairs up to $0.3 \mathrm{~mm}$ long, decaying into fibers, yellowish to brownish; basal leaf-blades (5.8-)7-13(-15.5) cm long $\times 1.5-3.7 \mathrm{~cm}$ wide, flat to rolled when dried, not rigid, glabrous abaxially, sometimes densely pubescent, pubescent adaxially, also with hairs on the margins (0.05-) $0.2-0.8(-1) \mathrm{mm}$ long, greenish-greyish to brownish; top culm leaf-sheaths $12-13.5(-16) \mathrm{cm}$ long, shorter than the internodes, rarely longer, glabrous, without cilia on the margins; top culm leafblades (9-)10-20 cm long $\times(-0.6) 0.8-8 \mathrm{~cm}$ wide, flat, usually parallel to the culm, with the central nerve similar to the lateral 


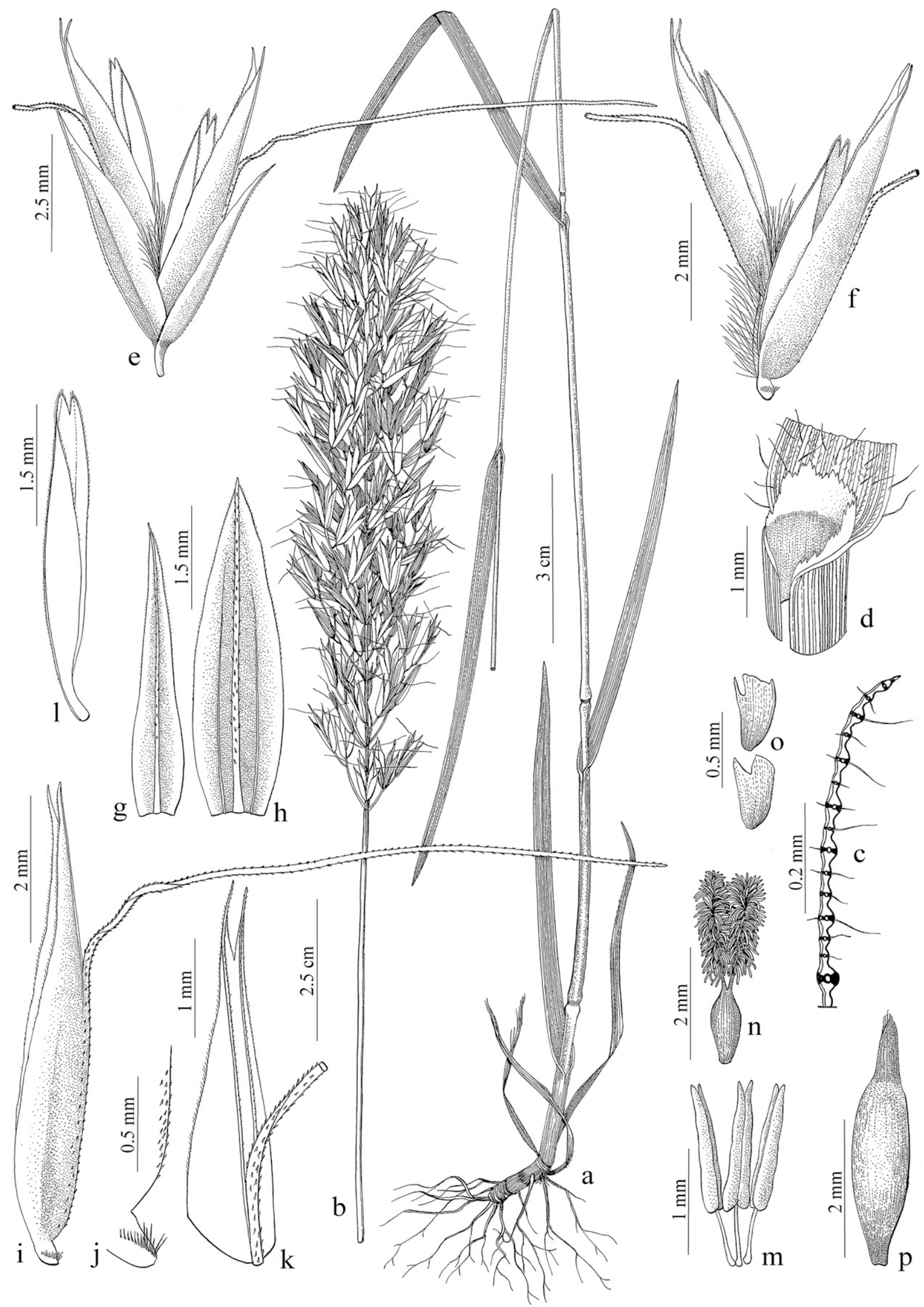

Fig. 10. Trisetum aeneum. a. Basal habit. b. Inflorescence. c. Transverse section of leaf-blade. d. Sheath, ligule, and portion of the blade. e. Spikelet. f. Florets. g. Lower glume, dorsal view. h. Upper glume, dorsal view. i. Floret. j. Callus, lateral view. k. Lemma, upper part, lateral view. 1. Palea, lateral view. m. Stamens. n. Pistil. o. Lodicules. p. Caryopsis. (Royle s. n., K-808692, a-b; Polunin et al. 281, UPS-V-644757, c-d; Jaquemont 1214, P-2243981, e-1; Duthie 12548, W, m-p; Stewart 18700, US-2044501, o). 
ones, with short adpressed hairs along the nerves abaxially, pubescent with longer hairs alternate with short ones adaxially, hairs (0.05-)0.1-1.2(-1.6) $\mathrm{mm}$ long, with short hairs on the margins, greenish to greyish; inner collar region glabrous; ligules 2.2-3.5 mm long, laciniate, glabrous. Basal node of the panicle glabrous. Panicles $7-15 \mathrm{~cm}$ long $\times$ $3-5 \mathrm{~cm}$ wide, lanceolate, rarely oblong to elliptic in outline, lax, rarely slightly dense with the rachis visible; rachis glabrous; branches single or paired, longest basal ones (1.4-)3-9(-9.3) cm long. Spikelets 6.5-9.2-(-10.5) $\mathrm{mm}$ long $\times(2.7-) 4.7-5.2 \mathrm{~mm}$ wide, 1-2(3)-flowered; pedicels (3.3-)3.6-6(-6.2) $\mathrm{mm}$ long, slightly pubescent, up to $0.1 \mathrm{~mm}$ long. Glumes unequal (ratio of lower glume length/upper glume length $=(0.64-) 0.7-0.8)$; lower glume $4.5-5.4$ (-5.7) $\mathrm{mm}$ long $\times(0.7-) 0.9-1.2 \mathrm{~mm}$ wide, narrowly lanceolate, rarely narrowly elliptic (ratio of lower glume width/lower glume length $=(0.14-) 0.17-0.22)$, acuminate, sometimes acute, 1(3)-nerved, glabrous, with hairs on the midrib and margins up to $0.08 \mathrm{~mm}$ long, greenish to greyish or yellowish; upper glume 6-7.6 mm long $\times$ 1.2-1.6 $(-1.8) \mathrm{mm}$ wide, narrowly lanceolate to narrowly elliptic (ratio of upper glume width/upper glume length = 0.16-0.25), acuminate, rarely acute, 3-nerved, glabrous, with hairs up to $0.1 \mathrm{~mm}$ long from the middle to the upper part of the central nerve and upper part of the margins, greenish, sometimes yellowish or greyish; rachilla segments between first and second floret 1.6-2.2 $\mathrm{mm}$ long, with hairs 0.9-1.5(-2) $\mathrm{mm}$ long; rachilla segments to sterile floret (0.3-)1.5-1.8 mm long, with hairs $0.3-2 \mathrm{~mm}$ long. Lemmas (6.5-)7.4-7.8(-8.4) $\mathrm{mm}$ long $\times(0.7-) 0.9-1.2 \mathrm{~mm}$ wide, narrowly elliptic to narrowly lanceolate, rarely oblong (ratio of lemma width/lemma length $=0.2-0.3$ ), scabridulous on the central part, with hairs up to $0.1 \mathrm{~mm}$ long, golden brown; callus $0.3-0.4 \mathrm{~mm}$ long, elliptic, glabrous; apical teeth (0.2-)0.4-1.2 $\mathrm{mm}$ long, with aristules 0.5-1.3 mm long; awn (10.5-)11-14(-15) mm long, inserted 4-4.8 $\mathrm{mm}$ from the base (ratio of awn insertion from the base length/lemma length $=0.56-0.63$ ), geniculate below the middle, twisted up to the geniculate part, with adpressed hairs up to $0.05 \mathrm{~mm}$ long. Paleas (4.2-) 4.6-5.2 mm long $\times 1.2-1.8 \mathrm{~mm}$ wide (ratio of palea length/ lemma length $=0.6-0.68$ ), elliptic to oblong, rarely lanceolate, margins with short antrorse hairs; teeth $0.3-0.6 \mathrm{~mm}$ long, with antrorse hairs up to $0.6 \mathrm{~mm}$ long from the middle to the upper part. Lodicules $0.7-0.9 \mathrm{~mm}$ long, irregularly or regularly 2-3 lobulate. Anthers $0.9-1.5 \mathrm{~mm}$ long. Ovary 0.8-1.8 mm long. Caryopsis $3 \mathrm{~mm}$ long $\times 0.6-0.7 \mathrm{~mm}$ wide, narrowly elliptic to elliptic. Figure 11.

Chromosome Number-Unknown.

Phenology_Flowering and fruiting from May to September.

Distribution and Habitat-This species occurs from central Nepal, Sikkim, and Bhutan, to south-central China (Eastern Yunnan and Sichuan); 2,800-5,000 $\mathrm{m}$ of elevation; in alpine meadows and shrubs, and river banks. Figure 9.

Specimens Examined-BHUTAN. Upper Mo Chu Dist, $28^{\circ} 7^{\prime} \mathrm{N}, 89^{\circ} 44^{\prime} \mathrm{E}$, 20 Sep 1984, Sinclair 5199 \& Long (K).

CHINA. Sichuan: Daocheng county, $28^{\circ} 54^{\prime} \mathrm{N}, 100^{\circ} 8^{\prime} \mathrm{E}, 1$ Aug 1982, Dong

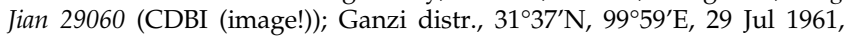
Junsheng 9847 (PE (image!)); Ji pu lang, Zhengxi farm, 28 $34^{\prime} \mathrm{N}, 100^{\circ} 59^{\prime} \mathrm{E}, 9$ Sep 1983, Qing 13824 (PE); $4.5 \mathrm{~km}$ northeast from the city Langtang, $29^{\circ} 52^{\prime} \mathrm{N}, 9^{\circ} 12^{\prime} \mathrm{E}, 27$ Aug 1983, Tang 1836 (PE); Samake, na ta, chang tai, bai yu town, $30^{\circ} 31^{\prime} \mathrm{N}, 100^{\circ} 17^{\prime} \mathrm{E}, 18$ Aug 1982, Tang 768 (PE). Tibet: Nanmulin county, Rendui distr., $30^{\circ} 4^{\prime} \mathrm{N} 89^{\circ} 6^{\prime} \mathrm{E}, 2$ Sep 1975, Qinghai-Tibet Team 7430 (KUN (image!), PE, QTPM (images!)). Yunnan: au-dessus du Col de Yen Tze hay, $25^{\circ} 35^{\prime} \mathrm{N}, 100^{\circ} 12^{\prime} \mathrm{E}, 6$ Aug 1888 , Delavay $3686(\mathrm{P})$; Lijiang county,

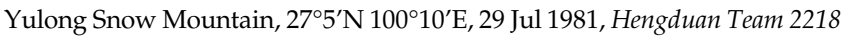
(PE (image!)); Qiaojia county, $27^{\circ} 1^{\prime} \mathrm{N} 103^{\circ} 6^{\prime} \mathrm{E}, 13$ May 1973, Kunming Group 8 (KUN (image!)).

INDIA. Sikkim: Northeast Sikkim, 14 Jun 1894, Cummins s. n. (K); Lonok Valley, $27^{\circ} 20^{\prime} \mathrm{N}, 88^{\circ} 40^{\prime} \mathrm{E}, 28$ Jul 1903, Younghusband 187 (K).

NEPAL. Central Region, Langtang, $28^{\circ} 10^{\prime} \mathrm{N}, 8^{\circ} 20^{\prime} \mathrm{E}, 16$ Sep 1986 , Miehe 11878 \& Miehe (K); Central Region, ob. Langtang, $28^{\circ} 10^{\prime} \mathrm{N}, 85^{\circ} 20^{\prime} \mathrm{E}$, 19 Sep 1986, Miehe 12324 \& Miehe (K); Central Nepal, Bagmati Zone, Rasuwa Distr., Langtrang Khola-Pirgona-Pyung-a pass-Base Camp, $28^{\circ} 12^{\prime} \mathrm{N}$, 86³8'E, 20 Jul 1992, Miyamoto 20288 (AAH); Eastern Region, Tamur Valley, Ghunsa, E of Lalungchung Gola, $27^{\circ} 30^{\prime} \mathrm{N}, 8^{\circ} 31^{\prime} \mathrm{E}, 27 \mathrm{Jul}$ 1956, Stainton 1143 (K).

Notes-Trisetum scitulum is easily differentiated from the rest of the species by the combination of slender habit, lax panicles, and long awns.

In his Flora of British India, Hooker (1897) identified as Avena flavescens material that corresponds to Trisetum scitulum, indicating the different characters in the description: "spikelets larger than in most other species of this section, the awn longer; rachilla nodose below the fl. gls., penicillate with long hairs." Bor (1956) dealt with the problems of Hooker's misapplied concept of the species, and gave a new name to it, T. scitulum, without describing it, only making reference to Hooker's English description of T. flavescens. Chrtek (1968) validated the name with a Latin description, and also citing this species from China for the first time.

As happened with T. turcicum, Chrtek (1968) included Trisetum scitulum in ser. Trisetum, because of its geniculate awn. In this work, for the same reasons indicated for T. turcicum, T. scitulum has been included in T. sect. Sibirica. Moreover, in recent molecular works (Barberá et al. unpublished), T. scitulum appears in T. sibiricum s. 1. clade, with the rest of species included in this section.

DOUBTFUL AND EXCLUDED NAMES-

Aira ruprechtii Griseb. ex Hook. f., Trans. Linn. Soc. London 23: 346. 1862, nom. inval., pro syn.

Trisetum flavescens var. genuinum Hack., Bull. Herb. Boiss. 7: 702.1899, nom. inval. (Art. 24.3)

Trisetum sibiricum var. alpinum Reverd., Fl. Krasnoyarsk Kraya 2: 49. 1964, nom. inval. (Art. 39.1)

Trisetum sibiricum var. bifidum (Thunb.) Makino ex T. Koyama, Grass. Jap. Neighb. Reg.: 533. 1987, nom. inval.

Trisetum sibiricum subsp. glabrum Galanin, Fl. Daurii 2: 125 (\& 138). 2009, nom. inval. (Art. 39.1)

Trisetum sibiricum f. litorale Rupr., Beitr. Pflanzenk. Russ. Reiches 2: 65. 1845, nom. nud.

ACKNOWLEDgments. This work was financed by Flora iberica CGL2014-52787-C3-1-P, CGL2012-32914 and CGL2015-66161-P, projects of the Spanish Government, supported by FEDER funds. We are indebted to the curators and staff of the herbaria mentioned in Material and Methods for kindly providing specimens. We thank the editor and anonymous reviewers for their critical reviews of the manuscript and for their helpful suggestions during the editing process. We thank Ramón Morales for translations of manuscripts in German. Toni Buira for his technical support. A. Quintanar for his revision of an earlier version of this manuscript. David Boufford for helping with Chinese translations. H. Nagamasu, Geraldine Reid, and Snežana Vukojičić helped in finding rare literature, type materials, and geographical localities. Roman Ufimov for Russian translations and helped in finding rare literature, and in many aspects. And for the rest of personnel from the Komarov Institute, N. N. Tzvelev, Dmitry Geltman, Elena Glazkova, and Denis Melnikov, for their help and their welcoming attitude during our stay. 


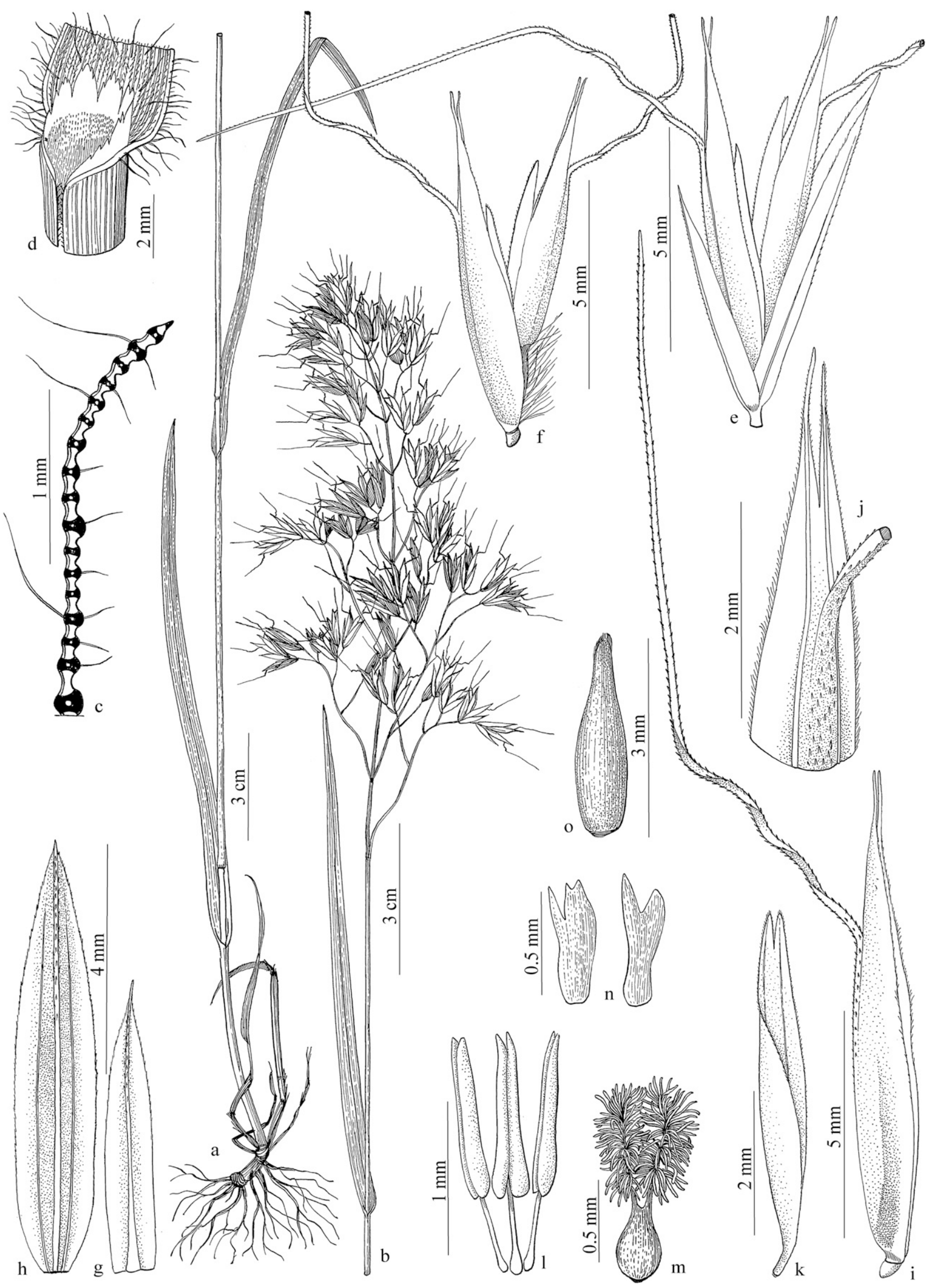

Fig. 11. Trisetum scitulum. a. Basal habit. b. Inflorescence. c. Transverse section of leaf-blade. d. Sheath, ligule, and portion of the blade. e. Spikelet. f. Florets. g. Lower glume, dorsal view. h. Upper glume, dorsal view. i. Floret. j. Lemma, upper part, lateral view. k. Palea, lateral view. l. Stamens. m. Pistil. n. Lodicules. o. Caryopsis. (Delavay 3365, P-2255919, a-b, d-m, o; Stainton 1143, K, c, n-o). 


\section{Literature Cited}

Anonymous. 1976. Flora Tsinlingensis 1. Beijing: Academia Sinica Press [in Chinese].

Barberá, P., C. Romero-Zarco, and C. Aedo. 2017. Taxonomic revision of Trisetum section Acrospelion (Poaceae: Pooideae: Aveninae) from Eurasia. Systematic Botany 42: XXX-XXX.

Bor, N. L. 1956 Notes on Asiatic Grasses: XXV. A Himalayan Trisetum. Kew Bulletin 11-212.

Chrtek, J. 1965. Bemerkungen zur Gliederung der Gattung Trisetum Pers. Botaniska Notiser 118: 210-224.

Chrtek J. 1966. Beitrag zur Kenntnis einiger Arten der Gattung Trisetum der Türkei. Botaniska Notiser 119: 486-490.

Chrtek, J. 1968. Bemerkungen zu Einigen Arten der Gattung Trisetum aus dem Sikkim-Gebiet. Acta Universitatis Carolinae. Biologica 1967: 103-107.

Clayton, W. D. and S. A. Renvoize. 1986. Genera Graminum, grasses of the world. Kew Bulletin. Additional series 13: 1-389.

Edgar, E. 1998. Trisetum Pers. (Gramineae: Aveneae) in New Zealand. New Zealand Journal of Botany 36: 539-564.

Ellis, R. P. 1976. A procedure for standardizing comparative leaf anatomy in the Poaceae I. The leaf-blade as viewed in transverse section. Bothalia 12: 65-109.

Ellis, R. P. 1979. A procedure for standardizing comparative leaf anatomy in the Poaceae II. The epidermis as seen in surface view. Bothalia 12: 641-671.

Enushchenko, I. 2011. On the genus Trisetum Pers. (Poaceae) in the Northern Asia. Novitates Systematicae Plantarum Vascularum 42: 50-62.

ESRI. 2008. ArcGIS version 9.3. Redlands, California: Environmental Systems Research Institute.

Finot, V. L., P. M. Peterson, R. J. Soreng, and F. O. Zuloaga. 2004. A revision of Trisetum, Peyritschia, and Sphenopholis (Poaceae: Pooideae: Aveninae) in Mexico and Central America. Annals of the Missouri Botanical Garden 91: 1-30.

Finot, V. L., P. M. Peterson, R. J. Soreng, and F. O. Zuloaga. 2005a. A Revision of Trisetum and Graphephorum (Poaceae: Pooideae: Aveninae) in North America, North of Mexico. Sida 21: 1419-1453.

Finot, V. L., P. M. Peterson, F. O. Zuloaga, R. J. Soreng, and O. Matthei. 2005b. A Revision of Trisetum (Poaceae: Pooideae: Aveninae) in South America. Annals of the Missouri Botanical Garden 92: 533-568.

Finot, V. L., C. M. Baeza, and O. Matthei. 2006. Micromorfología de la epidermis de la lemma de Trisetum y géneros afines (Poaceae, Pooideae). Darwiniana 44: 32-57.

Forbes, F. B. and W. B. Hemsley. 1904. An enumeration of all the plants known from China Proper, Formosa, Hainam, Corea, the Luchu Archipelago, and the Island of Hongkong, together with their distribution and synonymy. The Journal of the Linnean Society. Botany 36 $1-449$.

Frey, L. 1992. Taxonomy, karyology and distribution of the selected genera of tribe Aveneae (Poaceae) in Poland: II. Trisetum. Fragmenta Floristica et Geobotanica 37: 443-475.

GPWG (Grass Phylogeny Working Group). 2001. Phylogeny and subfamilial classification of the grasses (Poaceae). Annals of the Missouri Botanical Garden 88: 373-457.

Hooker, J. D. 1897. Avena. Pp. 274-280 in The Flora of British India. Vol. VII Cyperaceae, Gramineae, and general Index. Ed. 2. Delhi: Periodical Expert Book Agency.

Hsu, C. C. 1972. Preliminary chromosome studies on the vascular plants of Taiwan (V). Cytotaxonomy on some monocotyledons. Taiwania 17: 48-65.

Hultén, O. E. G. 1959. The Trisetum spicatum complex. Svensk Botanisk Tidskrift 53: 203-228.

Jonsell, B. 1980. Trisetum Pers. Pp. 220-224 in Flora Europaea vol. 5, eds. T. G. Tutin, V. H. Heywood, N. A. Burges, D. M. Moore, D. H. Valentine, S. M. Walters, and D. A. Webb. Cambridge, U. K.: Cambridge University Press.

Kitagawa, M. 1940. Materials to the Flora of Eastern Asia III. Reports of the Institute of Scientific Research Manghoukuo 4: 75-118.

Kitagawa, M. 1956. Notulae tractae ob floram Asiae orientalis (9). Shokubutsu Kenkyu Zasshi 31: 302-306.

Koch, S. D. 1979. The relationship of three Mexican Aveneae and some new characters for distinguishing Deschampsia and Trisetum (Gramineae). Taxon 28: 225-235.

Metcalfe, C. R. 1960. Anatomy of Monocotyledons. I. Gramineae. Oxford: Oxford University Press.

Nicora, E. G. 1978. Gramineae. Pp. 1-579 in Flora Patagónica 3, ed. M. N. Correa. Buenos Aires: Colección Científica del INTA.
Ono, H. and T. Tateoka. 1953. Karyotaxonomy in Poaceae I. Chromosomes and taxonomic relations in some Japanese grasses. Botanical Magazine Tokyo 66: 18-27.

Pančić, J. 1884. Floram Principatus Serbiae. Additamenta. Beograd: Državio Štamiariji).

Pignatti, S. 1982. Flora d'Italia vol. 3. Bologne: Edagricole.

Probatova, N. S. 1978. De Generum Trisetum Pers. et Koeleria Pers. Speciebus Caucasicis Notulae Systematicae. Novitates Systematicae Plan tarum Vascularium 15: 17-22.

Quintanar, A. and S. Castroviejo. 2010. Proposal to conserve Trisetum against Trisetaria (Gramineae). Taxon 59: 1602-1603.

Quintanar, A. and S. Castroviejo. 2013. Taxonomic revision of Koeleria (Poaceae) in the Western Mediterranean Basin and Macaronesia. Systematic Botany 38: 1029-1061.

Randall, J. L. and K. W. Hilu. 1986. Biosystematics studies of North American Trisetum spicatum (Poaceae). Systematic Botany 11: 567-578.

Rechinger, K. H. 1970. Flora des Iranischen Hochlandes und der Umrahmenden Gebirge: Persien, Afghanistan, Teile Von West-Pakistan, Nord Iraq, Azerbaidjan, Turkmenistan, Gramineae vol. 70. Graz: Akademische Druck.

Roshevitz, R. J. 1922. Note sur le Trisetum sibiricum Rupr. une espèce oubliée. Bulletin du Principal Jardin Botanique de la République Russe 21: 87-91.

Sãvulescu, T. 1972. Flora Reipublicae Socialisticae Romania vol. XII. Bucarest: Academia Republicii Socialiste România

Soreng, R. J., P. M. Peterson, G. Davidse, E. J. Judziewicz, F. O. Zuloaga T. S. Filgueiras, and O. Morrone. 2003. Catalogue of the New World grasses (Poaceae): IV. Subfamily Pooideae. Contributions from the United States National Herbarium 48: 1-730.

Soreng, R. J., J. I. Davis, and M. A. Voionmaa. 2007. A phylogenetic analysis of Poaceae tribe Poeae sensu lato based on morphological characters and sequence data from three plastid-encoded genes: Evidence of reticulation, and new classification for the tribe. Kew Bulletin 62: 425-454.

Soreng, R. J., P. M. Peterson, K. Romaschenko, G. Davidse, F. O. Zuloaga, E. J. Judziewicz, T. S. Filgueiras, J. I. Davis, and O. Morrone. 2015. A worldwide phylogenetic classification of the Poaceae (Gramineae). Journal of Systematics and Evolution 53: 117-137.

Steudel, E. G. 1854. Synopsis plantarum glumacearum. Pars I. Gramineae. Stuttgart: J. B. Metzler.

Stuessy, T. F., D. J. Crawford, D. E. Soltis, and P. Soltis. 2014. Plant systematics. The origin, interpretation, and ordering of plant biodiversity. Königstein: Koeltz Scientific Books.

Tateoka, T. 1978. Cytotaxonomic conspectus of Japanese Trisetum (Poaceae). Bulletin of the National Science Museum, Tokyo. Series B. Botany 4: $1-3$.

Thiers, B. 2017. Index Herbariorum: A global directory of public herbaria and associated staff. New York Botanical Garden's Virtual Herbarium. http://sweetgum.nybg.org/science/ih/.

Tolivia, D. and J. Tolivia. 1987. Fasga: A new polychromatic method for simultaneous and differential staining of plant tissues. Journal of Microscopy 148: 113-117.

Tzvelev, N. N. 1976. Zlaki SSSR. Saint Petersburg: Nauka Publishers [English translation: 1983. Grasses of the Soviet Union. New Delhi: Amerid Publishing.]

Tzvelev, N. N. 1989. The system of grasses and their evolution. Botanical Review 55: 141-204.

Van Royen, P. 1980. The Alpine flora of New Guinea vol. 2. Vaduz: J. Cramer.

Veldkamp, J. F. and J. C. van der Have. 1983. The genus Trisetum (Gramineae) in Malesia and Taiwan. Gardens' Bulletin (Singapore) 36: 125-135.

Watson, L. and M. J. Dallwitz. 1992. The grass genera of the world. Wallingford: C. A. B. International.

Wölk, A. and M. Röser. 2013. The genus Trisetopsis and new combinations in oat-like grasses (Poaceae). Schlechtendalia (Halle) 25: 57-61.

Wölk, A. and M. Röser. 2017. Hybridization and long-distance colonization in oat-like grasses of South and East Asia, including an amended circumscription of Helictotrichon and the description of the new genus Tzveleviochloa (Poaceae). Taxon 66: 20-43.

Wu, Z. L. and S. M. Phillips. 2006. Poaceae. Pp. 1-752 in Flora of China Vol. XXII, eds. W. Zhengyi, P. H. Raven and H. Deyuan. Beijing and St. Louis: Science Press and Missouri Botanical Garden.

Zhukova, P. G. 1967. Chromosome numbers in some species of plants of the North-Eastern part of the U.S.S.R., II. Botanicheskii Zhurnal 52: 983-987.

APPENDIX 1. Index to numbered collections cited. The numbers in parentheses refer to the corresponding species in the text. 
Alanko et al. 52917 (1.a); Aleksandrova, V. D. et L. N. Tiulina 134 (1.b), 242 (1.b); Andreev, V. N. 1418 (1.b), $4906 a$ (1.a), $4906 b$ (1.a); Andreev, V. N. et Z. Savkina 692 (1.b), 898 (1.b); Balansa 848 (2), 1551 (2); Beattie, R. et Y. Kurihara 10622 (3), 10832 (3); Bergman, S. 237 (1.b); Blagoveshchenskiy, N. V. 532 (1.a); Blagoveshchenskiy, N. V. et G. I. Poplavskaia 1248 (1.a); Bor, N. L. 15560 (5); Borodin, I. 278 (1.a); Boufford, D. E. et E. W. Wood 18987a (3); Brass, L. J. 9118 (3); Bunge, A. 101 (1.a); Bush et Bush 34 (2), 42 (2); Cajander, A. K. 2074 (1.b); Cherskiy, A. I. 86 (1.a); Chien-chang Hsu et Kuo 5394 (3), 7293 (3); Ching, R. C 8767 (4); Clarke, C. B. 31034 (5); Davis, P. H. 46563 (2); Davis, P. H. et Polunin 24763 a (2); Delavay, J. M. 3686 (6); Desiatkin, N. L. 502 (1.a); Deyl, M. et J. Sojak 344 (1.a), 2917 (1.a), 3268 (1.a); Diukina, N. 539 (1.a); Dong Jian 29060 (6); Dorofeev, V. I. 73 (1.a); Dorsett, P. H. et Morse, W. J. 483 (3); Dragulenko, G. V. 26/1 (1.a); Drobov, V. P. 384 (1.a); Duthie, J. F. 12548 (5), 12562 (5), 13646 (5) Egorova, T.V. et al. 1685 (2), 1844 (2); Elias, T. S. et al. 4847 (1.a); Evstifeeva, M. 360 (1.a); Faurie, U. J. 499 (3), 689 (3), 804 (1.a), 805 (1.a), 807 (3), 883 (3), 1212 (3), 1246 (1.a), 2228 (3), 2359 (3), 4468 (3), 4469 (3), 5214 (3), 5711 (3), 7191 (3), 8104 (3), 8456 (1.a), 8504 (1.a), 10523 (1.a), 10524 (1.a); Forestry Administration Henan 465 (4); Franchel, A. 1472 (3); Fu, K. T. 1386 (1.a), 5281 (1.a); Furuse, M. 9171 (1.a), 9176 (1.a), 47899 (1.a); Gage, S. SG1427 (1.b), SG1976 (1.b), SG2021 (1.b), SG2117 (1.b), SG4139 (1.b), SG4274 (1.b), SG4492 (1.b); Gage, S. et B. Semsrott SG1814 (1.a); Gjaerevoll, O. 294 (1.b); Golovina, E.O. 310 (1.a); Gomolitskiy, P. et T. Semenikhina 716 (1.a); Gusev, V. A. 256 (1.a); Handel-Mazzetti, H. F. 2279 (2); Hashimoto, CH. 9721 (1.a); He Yinyu 25309 (4); Henan Team 1023 (4), 1179 (4); Hengduan Team 2218 (6); Henry, A. 2558 (4), 6643 (4), 6706 (4); Hitchcock, A.S. 18318 (3), 18344 (3), 18534 (4), 18551 (4), 18558 (4); Huang Deng, Y. Y. 4031 (3); Hultén, E. 798 (1. a); Iarygin, I. I. 147 (1.a); Ikonnikov-Galitzky, N. 119 (1.a); IkonnikovGalitzky, N. P. et V. A. Ikonnikov-Galitzky 3426 (1.a); Iliin, M. et B. Ovchinnikov 28 (1.a); Iltis, H. H. et al. 561 (1.a), 1321 (1.a); Isachenko, T. I. et al. 219 (1.a); Junsheng, Y. 9847 (6); Karev, G. I. 669 (1.a); Karo, F. 270 (1.a); Keng, Y. L. 2314 (3), 2977 (3); Khanmimchun, V. et V. Amelchenko 2207 (1.a); Khitun, O.V. 8120 (1.b), 8151 (1.b), 8234 (1.b); Khutov, N. A. 130 (1.a); Kirino, S. 652 (3); Klements, E. 275 (1.a); Koelz, W. 22048 (5); Kondo, K. et T. Kurosawa 227 (3); Konta, F. et K. Murata 1184 (3); Koponen, T. 15299 (3); Korchagin, A. et O. Gaze 169 (1.a); Koroleva, T. et V. Petrovsky 5703 (1.b); Kozhevnikov, Y. P. 154 (1.b); Kuldiushevskiy, I. D. 110/2 (1.a); Kunming group 8 (6); Kurosawa, T. 5063 (3); Kurosawa, T. et al. 10102 (3); Kusnezow, N. I. et W. W. Reverdatto 2276 (1.b), 4043 (1.b); Kuznetsov, I. V. 78 (1.a);
Legendre, A. F. 391 (1.a); Legler, B. 773 (1.a), 1246 (1.a); Levinge, H. C. 27424 (5); Liou, T. N. et al. 2042 (4); Lipshits, S. Y. 249 (1.a); Lipshits, V. 3044 (1.a); Lishuxin 3118 (1.a); Litvinov, D. 768 (1.a), 898 (1.a); Mameev, S. 77 (1.a), 675 (1.a); Martins, L. 2301 (1.a); Medvedeva, L. I. et al. 641 (1.a); Mengning Team 1262 (1.a); Miehe, G. et S. Miehe 3891 (5), 11878 (6), 12324 (6); Miyamoto, F. 20288 (6); Murata, K. et al. 11739 (3); Murata, J. et al. 1821 (3); Naito, T. et al. 15 (3); Nemoto, T.et al. 9574036 (1.a); Nestruev, S. S. 1871 (1.a); Noskov, A. K. et al. 291 (1.a); Ohwi, J. 58 (3), 378 (1.a), 2326 (3), 2720 (3), 5261 (1.a), 6739 (3), 8305 (1.a); Pančić, J. 1150 (1.a); Péronin, A. 214 (2); Polunin, O. et al. 281 (5); Poplawska, H. 1743 (1.a); Popov, M. G. 501 (1.a); Poretskiy, A. 298 (1.a); Porsild, A. E. 7122 (1.b); Porsild, A. E. et R. T. Porsild 893 (1.b), 1414 (1.b); Pospelov, I. N. 08-0466 (1.b), 97-376 (1.b); Qing, Z. T. 13824 (6); QinghaiTibet Team 7430 (6); Ream, R. R. 589 (3); Rebristaja, O. V. et S. A. Gokorevskich 553 (1.a); Rebristaya O. V. et Tokarevskikh 702 (1.b); Scamman, E. 5410 (1.b); Schikama, K. 209 (3); Selivanova-Gorodkova, E. 1521 (1.a); Semenikhina, T. 183 (1.a); Semsrott, B. BS0173 (1.b), BS1487 (1.b); Seregin, A. 3135 (1.a); Shan Hanrong et al. 8043 (4), 8389 (4), 9014 (4); Shanxi Team 246 (1.a), 1938 (1.a); Shaulo, D. et I. Belskaya 1561 (1.a); Shaulo, D. et D. Saya 1562 (1.a); Shiota, K. 5579 (3); Shipchinskiy, N.V. 145 (1.a), 529 (1.a); Sinclair, I. W. et D. G. Long 5199 (6); Sintenis, P. 1142 (2); Smirnov, V. I. 70 (1.a), 542 (1.a); Smirnov, P. A. 49 (1.a), 119 (2); Smith, H. 2772 (1.a), 3447 (1.a), 4276 (6), 7173 (1. a); Soják, J. et V. Vasak 7177 (1.a); Solstad, H. et Elven 04/0266A (1.b); Sovetkina, M. et S. Chausova 2706 (1.a); Stainton, J. D. 1143 (6); Stainton, J. D. et al. 3785a (5), 4648 (5); Steward, A. N. 2516 (3), 2559 (4); Stewart, R. R. 18448 (5), 18700 (5), 21829 (5); Stewart, R. R. et A. Rahman 25001 (5); Stileman, R. 17 (2); Strachey, R. et J. E. Winterbottom 1 (5); Sukachev, V. et G. Poplavskaya 2577 (1.a); Sukopp, U. 1523 (1.a), 1658 (1.a); Tang, T. 1433 (1.a); Tang, X. 768 (6), 1836 (6); Taquet, E. 1891 (1.a), 3403 (3), 3423 (1.a), 5096 (3); Tatli, A. 5331 (2); Temnoev, N. I. 62 (1.a); Tikhomirov, B.A. 73 (1.a); Tiulina, L. N. 223 (1.b); Tolmachev, A. I. 770 (1.b); Tolmachev, A. I. et al. 245 (1.b); Tomin, M. P. 153 (1.a); Transhel, V. 153 (1.a); Tsang, W. T. 20309 (3), 23765 (3); Tsiang, Y. et H. Wang 16458 (3); Tsugaru, S. et T. Takahashi 24822 (3), 25044 (3); Tsvelev, N. 266 (1.a); van Royen, P. 10867 (3); Vasak, V. 9247 (1.a); Veldkamp, J. F. 6248 (3); Wang, T. P. 4559 (1.a); Wenzhong Wang 141 (1.a); Wilford, C. 938 (3); Xiaoen Tian 1089 (1.a); Xiaozong Tang 1903 (1.a); Yao Kan 79429 (4); Yellow river Team 2552 (1.a), 2614 (1.a); Yokota, A. 958 (3); Yonekura, K. 448 (3); Yongsok, O. 7613 (3), 10030 (3); Younghusband, F. E. 187 (6); Yunatov, A. A. 361 (1.a), 15678 (1.a); Yunatov, A. A. et al. 1517 (1.a); Zhirov, I. 223 (1.a); Zinger, N. 796 (1.a). 